\title{
Computational Fluid Dynamics Modeling of Redundant Stent-Graft Configurations in Endovascular Aneurysm Repair
}

by

Leonard W. H. Tse

A thesis submitted in conformity with the requirements for the degree of Master of Applied Science (M.A.Sc.)

Institute of Biomaterials and Biomedical Engineering University of Toronto

(C) Copyright by Leonard W.H. Tse, 2010 


\title{
Computational Fluid Dynamics Modeling of Redundant Stent- Graft Configurations in Endovascular Aneurysm Repair
}

\author{
Leonard W. H. Tse \\ Master of Applied Science \\ Institute of Biomaterials and Biomedical Engineering \\ University of Toronto \\ 2010
}

\begin{abstract}
During endovascular aneurysm repair (EVAR), if the stent-graft device is too long for a given patient the redundant (extra) length adopts a convex configuration in the aneurysm. Based on clinical experience, we hypothesize that redundant stent-graft configurations increase the downward force acting on the device, thereby increasing the risk of device dislodgement and failure. This work numerically studies both steady-state and physiologic pulsatile blood flow in redundant stent-graft configurations. Computational fluid dynamics simulations predicted a peak downward displacement force for the zero-, moderate- and severe-redundancy configurations of $7.36,7.44$ and $7.81 \mathrm{~N}$, respectively for steady-state flow; and 7.35, 7.41 and $7.85 \mathrm{~N}$, respectively for physiologic pulsatile flow. These results suggest that redundant stent-graft configurations in EVAR do increase the downward force acting on the device, but the clinical consequence depends significantly on device-specific resistance to dislodgement.
\end{abstract}




\section{Acknowledgements}

A very wise surgeon-scientist warned me that "the only person in the world who will know how hard you work is your wife." This has become very evident over the last two years, and I would like to thank my wife Mira and my daughters Kaitlyn, Megan, and Abigail for their patience and sacrifice.

Sincere thanks to my supervisor, Cristina Amon, for her expertise, guidance, and for taking me on despite her busy schedule as the Dean. I thank her also for bringing in the other members of the lab who have contributed an essential and enormous amount to my work: Tina Shek and Aydin Nabovati, the illustrious members of the 'Fluids Team'. Dan Sellan for my orientation, getting me on my feet, and lots of fruitful discussion. Elizabeth Munro for her apple pie, coffee teachings, and all her help with my post-post-post-graduate career. Our student Alanna Yontef for her time and efforts.

K. Wayne Johnston for his guidance, and all the hallway advice. My committee chair David Steinman for his feedback to improve the quality of this work. Tony Easty for participating in this thesis. Peter Ballyk for the coffee shop advice during my visits to his hospital.

The members of the Division of Vascular Surgery at the Toronto General Hospital for all their support: personal, clinical, and financial - Barry Rubin, Thomas Lindsay, George Oreopoulos, Graham Roche-Nagle, and Doug Wooster. And to Bryce Taylor and Richard Reznick who helped make it possible.

Gilles Soulez and Oren Steinmetz for setting me on the academic path. Geoffrey, Agatha, Raymond and Stanley Tse for telling me to "go for it".

Tim McGloughlin for kindly supplying the coefficients for the physiologic velocity and pressure profiles.

This work was supported financially through the Natural Sciences and Engineering Research Council of Canada and the Canadian Society for Vascular Surgery. 


\section{Table of Contents}

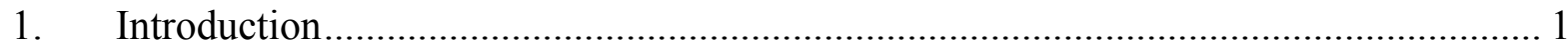

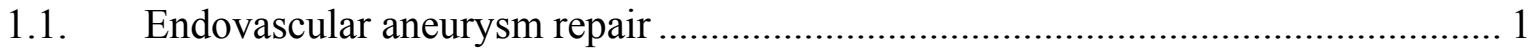

1.2. Redundant configurations ....................................................................... 3

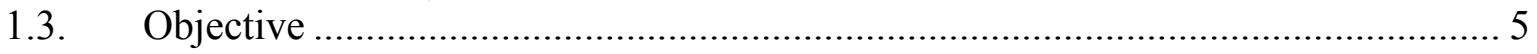

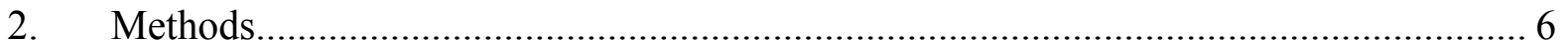

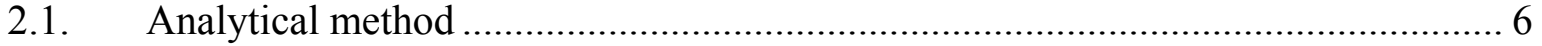

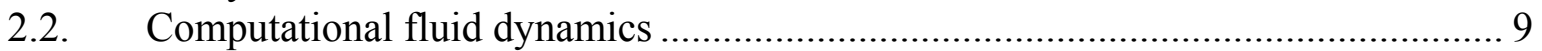

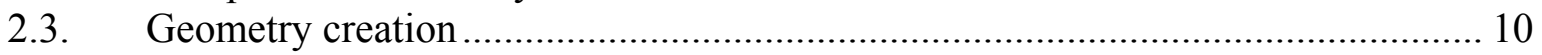

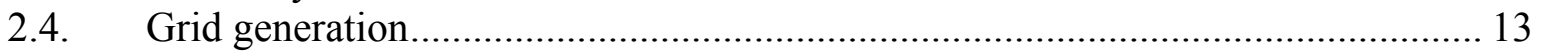

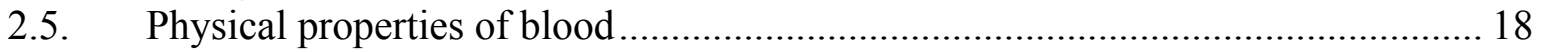

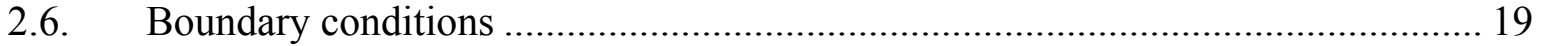

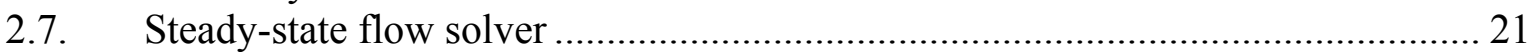

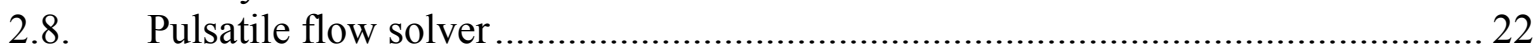

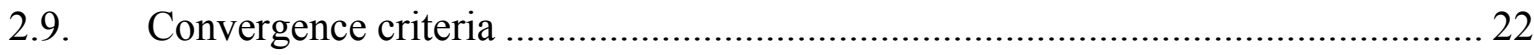

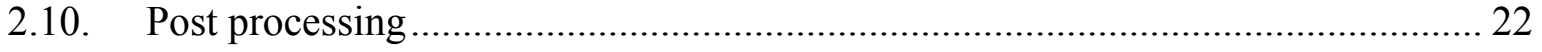

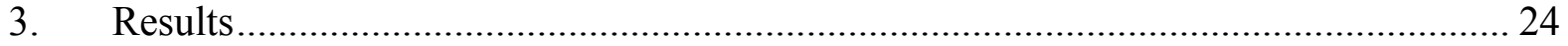

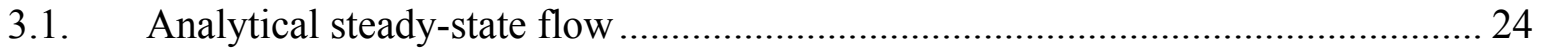

3.2. Computational steady-state flow .................................................................. 24

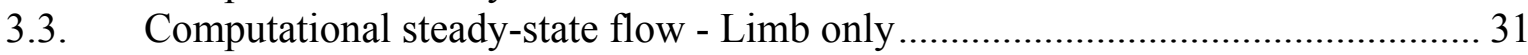

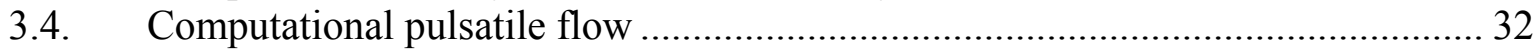

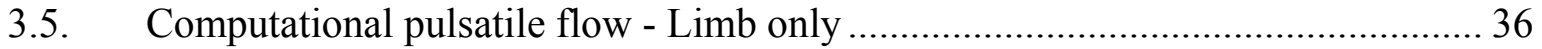

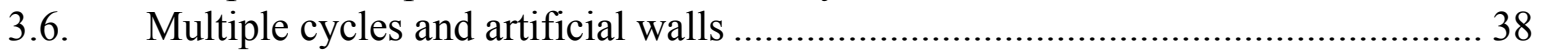

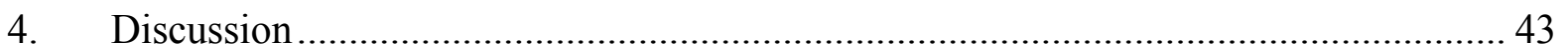

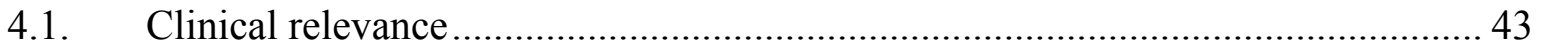

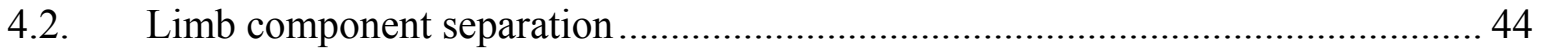

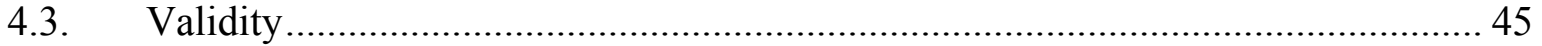

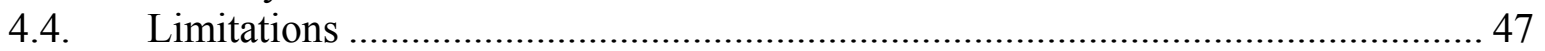

4.5. Outlet boundary conditions - equal pressure vs. equal mass flow ....................... 48

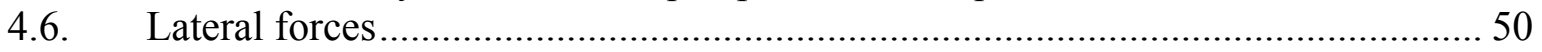

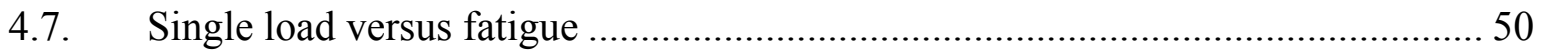

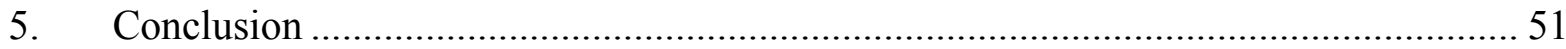

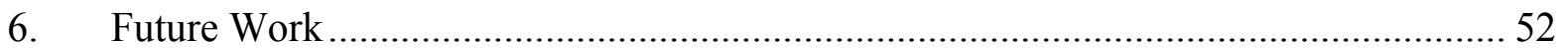

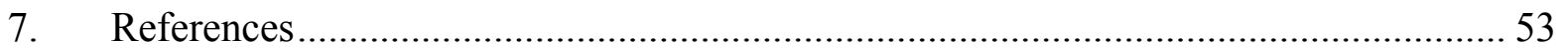




\section{List of Tables}

Table 1: Dimensions for stent-grafts used in CFD studies in the

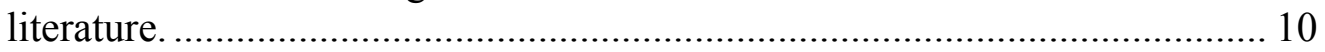

Table 2: Limb Component Separation Force for Steady-State

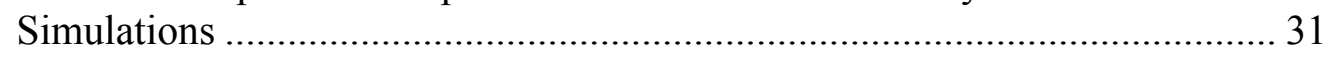

Table 3: $\quad$ Parameters and results of other CFD studies of stent-grafts............................ 46 


\section{List of Figures}

Figure 1: Illustration of abdominal aortic aneurysm and location in the body. *reproduced with permission from Medtronic, Inc. 1

Figure 2: Example of stent-graft deployed in abdominal aortic aneurysm. Image on the right is a magnified view of the connection between the main-body and the modular limb. *reproduced with permission from Medtronic, Inc.

Figure 3: $\quad$ Schematic of graft placement inside aneurysm demonstrating the origin of graft redundancy.....

Figure 4: Drawings of examples of grafts with redundant limbs in aneurysm sac.

Figure 5: Schematic of zero-redundancy (straight-limb, on left), moderate redundancy $\left(90^{\circ}\right.$-bend, in middle), and severeredundancy $\left(180^{\circ}\right.$-bend, on right) configurations used in the analytical approach.

Figure 6 Three representative three-dimensional geometries created for numerical study of zero-redundancy (left), moderateredundancy (middle), and severe-redundancy (right) configurations.

Figure 7: Creation of moderate-redundancy geometry with the same outlet angle and area as zero-redundancy geometry.

Figure 8: Example of tetrahedral meshing. Note that the surface is covered with triangles (one face of a tetrahedron).

Figure 9: Example of blocking (left side) used to create hexahedral mesh (right side).

Figure 10: Plot of spatial velocity profile of a $600 \mathrm{~mm}$ straight pipe for analytical solution (dotted line), hexahedral mesh (green circles), and tetrahedral mesh (red triangles).

Figure 11: Example of outlet, demonstrating finer mesh near walls and coarser near centre, while maintaining a hexahedral mesh.

Figure 12: Example demonstrating the mid-plane grid of the moderateredundancy limb where the flow is from the right to the left. The grid is finer near the walls, at the curve, and slightly downstream from the curve. 
Figure 13: Plot of computationally predicted downward force (in Newtons) versus number of nodes for severe-redundancy grids. The $1.9 \times 10^{6}$-node grid was used.

Figure 14: Plot of CPU time required for solution (in Hours) versus number of nodes for severe-redundancy grids. The $1.9 \times 10^{6}$ node grid was used.

Figure 15: Plot of physiologic velocity profile (temporal) used for pulsatile studies. The coefficients for this Fourier series were supplied by $\mathrm{T}$. McGloughlin through direct correspondence [28] and were those used in his previously published study [7].

Figure 16: Examples of three-dimensional velocity profiles at inlet during forward-flow systolic phase (left) and reversed-flow diastolic phase (right).

Figure 17: Downward displacement force for steady-state computational study for equal-mass flow (blue) and equal-pressure (red) outlet conditions

Figure 18: Equal outlet mass flow streamlines for zero-redundancy configuration. 26

Figure 19: Equal outlet pressure streamlines for zero-redundancy configuration.

Figure 20: Equal outlet mass flow streamlines for moderate-redundancy configuration.

Figure 21: Equal outlet pressure streamlines for moderate-redundancy configuration. Note there are slightly more streamlines being "diverted" to the left limb on the left.

Figure 22: Equal outlet mass flow streamlines for severe-redundancy configuration. Note the increased velocities (indicated by red colour) for the limb on the right.

Figure 23: Equal outlet pressure streamlines for severe-redundancy configuration. Note there are more streamlines being "diverted" to the limb on the left.

Figure 24: Velocity vectors on mid-coronal $(\mathrm{z}=0)$ plane for zeroredundancy configuration under equal-pressure condition. 30

Figure 25: Velocity vectors on mid-coronal $(\mathrm{z}=0)$ plane for moderateredundancy configuration under equal-mass flow (left) and 
equal-pressure (right) conditions. No recirculation zones near outlet boundary.

Figure 26: Velocity vectors on mid-coronal $(z=0)$ plane for severeredundancy configuration under equal-mass flow (left) and equal-pressure (right) conditions. Note recirculation zone downstream of curvature; no recirculation zones near outlet boundary; red arrows seen on left indicate velocities are greater (see legend in Fig. 24 for values).

Figure 27: Plot of downward displacement force (in Newtons) over time (in seconds) for severe-redundancy (dark blue squares), moderate-redundancy (light blue triangles), and zeroredundancy (pale blue circles) configurations for equal-mass flow outlet condition.

Figure 28: Plot of downward displacement force (in Newtons) over time (in seconds) for severe-redundancy (dark red squares), moderate-redundancy (light red triangles), and zeroredundancy (pale red circles) configurations for equal-pressure outlet condition.

Figure 29: Velocity vectors on mid-coronal $(z=0)$ plane for moderateredundancy configuration with equal-mass flow outlet condition at a) $0.30 \mathrm{~s}$ (peak systole), b) $0.35 \mathrm{~s}$ (early deceleration), c) $0.45 \mathrm{~s}$ (late deceleration), d) $0.55 \mathrm{~s}$ (flow reversal), and e) $1.10 \mathrm{~s}$ (late diastole).

Figure 30: Velocity vectors on mid-coronal $(z=0)$ plane for severeredundancy configuration with equal-mass flow outlet condition at a) $0.25 \mathrm{~s}$ (acceleration phase), b) $0.30 \mathrm{~s}$ (peak systole), c) $0.31 \mathrm{~s}$ (just after peak systole), d) $0.35 \mathrm{~s} \mathrm{(early}$ deceleration), e) $0.45 \mathrm{~s}$ (late deceleration), f) $0.55 \mathrm{~s}$ (reversal of flow), and g) $1.10 \mathrm{~s}$ (late diastole). 35

Figure 31: Plot of limb component separation force (in Newtons) over time (in seconds) for severe-redundancy (dark blue squares), moderate-redundancy (light blue triangles), and zeroredundancy (pale blue circles) configurations for equal-mass flow outlet condition.

Figure 32 Plot of limb component separation force (in Newtons) over time (in seconds) for severe-redundancy (dark red squares), moderate-redundancy (light red triangles), and zeroredundancy (pale red circles) configurations for equal-pressure outlet condition. 
Figure 33: Plot of downward force for two cardiac cycles simulated for the moderate-redundancy configuration that are shown overlapped. Note the small difference at the beginning of the cycle that quickly disappears.

Figure 34: Plot of downward force for equal outlet mass flow with artificial walls (red squares) and without artificial walls (green circles) when there is flow reversal at the outlets.

Figure $35 \quad$ Velocity vectors on the outlet boundary, with artificial walls (left panels) and without artificial walls (right panels) during initiation of the flow reversal phase $(\mathrm{t}=0.52 \mathrm{~s}$, upper panels) and during full flow reversal $(t=0.55 \mathrm{~s}$, lower panels). The solver had erected artificial walls over $56 \%$ of the outlet boundary during its first iteration of $\mathrm{t}=0.52 \mathrm{~s}$. One can clearly see that during full flow reversal at $\mathrm{t}=0.55 \mathrm{~s}$, there is inappropriate forward-directed flow in the model that used artificial walls

Figure 36: Plot of values for root mean square (RMS) of residuals for equal outlet mass flow with artificial walls (red squares) and without artificial walls (green circles) when there is flow reversal at the outlet.

Figure 37: Graph comparing anchoring force (in Newtons) for various proximal fixation strategies for stent grafts, from references [32-34].

Figure 38: Graph of experimentally-derived forces required to separate limb components for various commercial devices. $(\mathrm{C})=$ from Cina et al. [36] $(\mathrm{H})=$ Hinchliffe et al. [34].

Figure 39: Graph comparing results from Matlab model with published results $[5,6]$.

Figure 40: Example of $5^{\circ}$ difference in angle of outlet. The zeroredundancy limb (green) is overlapped onto the erroneous moderate-redundancy limb (grey).

Figure 41: Plot of downward displacement force over time. Note the moderate-redundancy configuration (blue triangles) is consistently lower throughout the cardiac cycle.

Figure 42: $\quad$ Outlets re-aligned. 63 


\section{List of Appendices}

Appendix I: Derivation of equations

Appendix II: Comparing model with published analytical results _............................... VIII

Appendix III: Example of mismatched outlet angle _................................................. IX

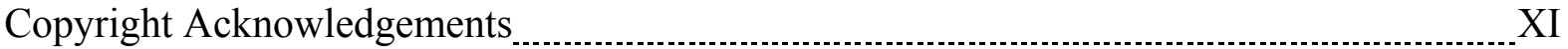




\section{List of Abbreviations}

\begin{tabular}{|l|l|}
\hline AAA & abdominal aortic aneurysm \\
\hline ASME & American Society of Mechanical Engineers \\
\hline CFD & computational fluid dynamics \\
\hline EVAR & endovascular aneurysm repair \\
\hline IMECE & International Mechanical Engineering Congress \& Exposition \\
\hline MRI & Magnetic Resonance Imaging \\
\hline RMS & Root Mean Square \\
\hline
\end{tabular}




\section{Introduction}

\subsection{Endovascular aneurysm repair}

One of the most significant achievements in surgery has been the ability to repair abdominal aortic aneurysms (AAAs). An aneurysm is a pathologic dilatation of a weakened artery. The most commonly affected segment is the abdominal portion of the aorta (main artery trunk of the body). Figure 1 illustrates an abdominal aortic aneurysm and its typical location. Approximately 5\% of men over the age of 65 have an AAA [1]. The natural course of an AAA is to progressively dilate, which may result in artery rupture and death. The mortality rate associated with ruptured AAAs is well over $80 \%$.

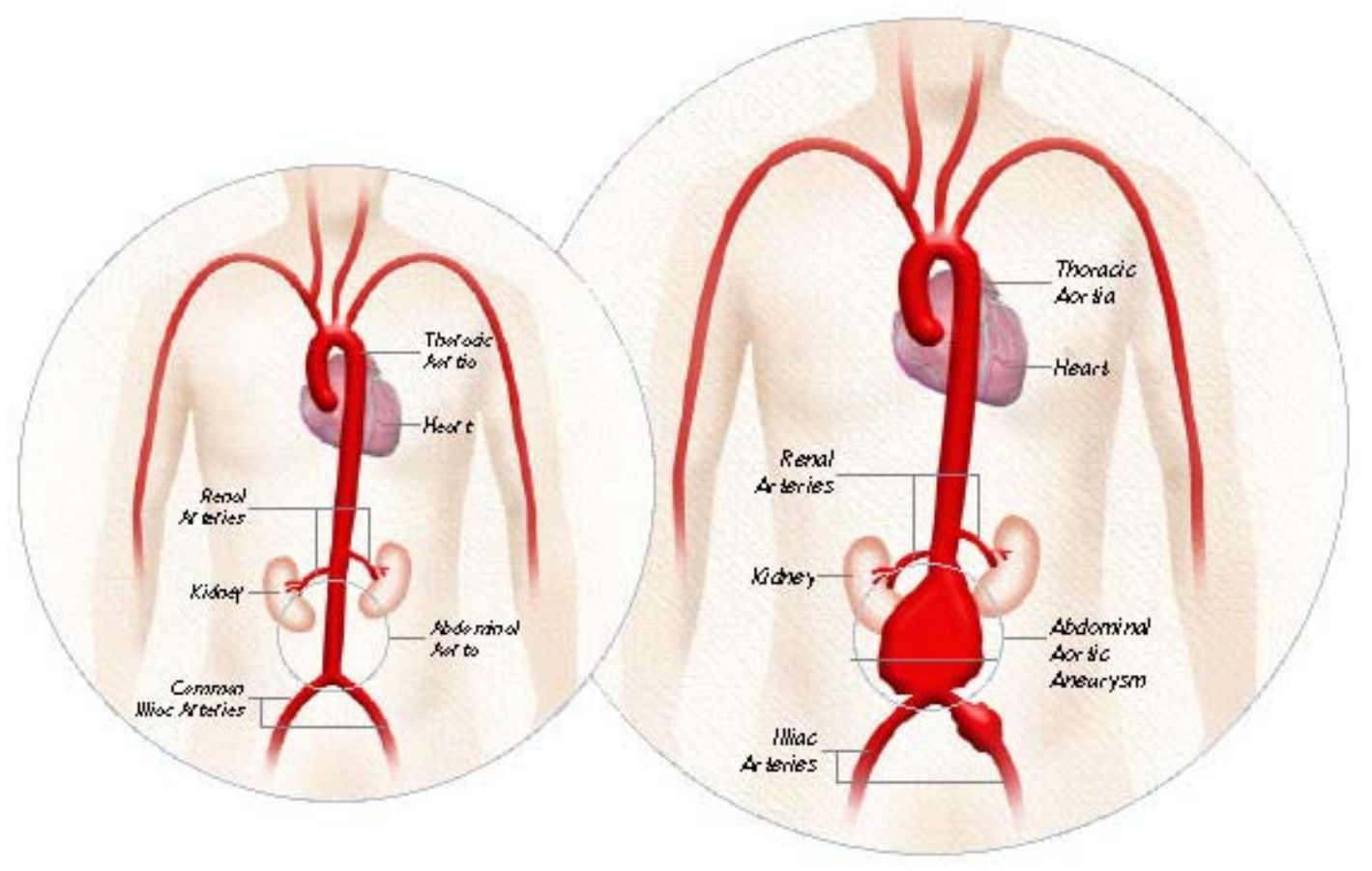

Figure 1: Illustration of abdominal aortic aneurysm and location in the body. *reproduced with permission from Medtronic, Inc. 
Since the 1950's, the conventional method to repair AAAs has been replacement of the weakened segment with a polyester fabric tube, through a large abdominal incision. Even prior to this, there were attempts at minimally-invasive treatments, such as coiling large lengths of wire into aneurysms in attempt to arrest blood flow within them. However, the first real minimally-invasive repairs were only described by Volodos et al. in 1986 [2], and introduced to the "Western World" by Parodi et al. in 1991 [3]. Endovascular aneurysm repair (EVAR) is a minimally-invasive technique whereby the replacement fabric tube (the graft) is compressed, inserted through the groin, and delivered to the aneurysm site from within the arteries. The graft is pushed open by a metallic scaffold (the stents). The stentgraft then confines the blood within it and relieves the pressure on the weakened aneurysm wall. In order to accommodate the aortic bifurcation, most modern devices are modular so that the device can be put together in situ and extended into each iliac limb. Figure 2 is an illustrative example of a stent-graft that has been implanted into an aneurysm. The modular connection is seen to the right of the illustration, where the top portion of the modular limb has been deployed inside the main-body and is held in place by friction-fit.

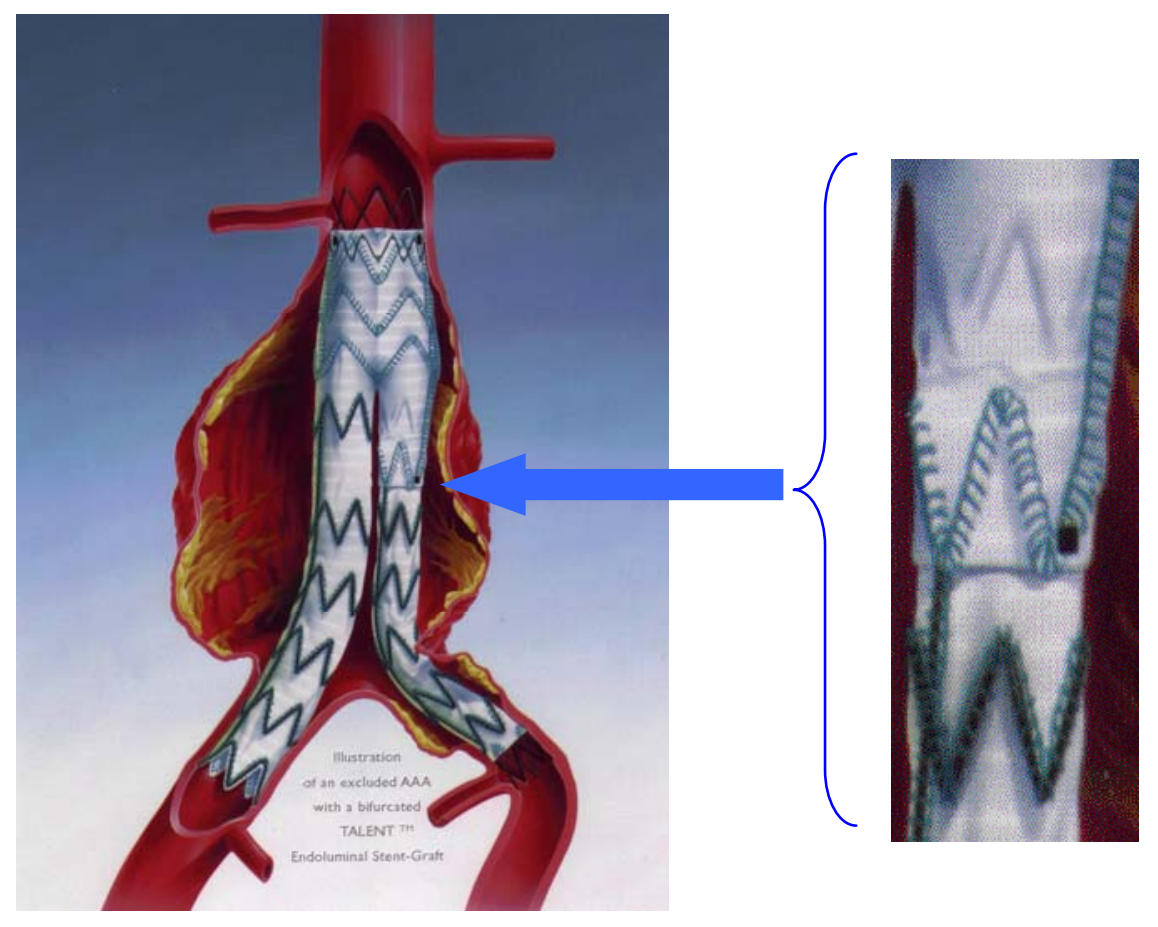

Figure 2: Example of stent-graft deployed in abdominal aortic aneurysm. Image on the right is a magnified view of the connection between the main-body and the modular limb. *reproduced with permission from Medtronic, Inc. 
This approach is much less traumatic than open surgery, and can be performed percutaneously (without incisions) or through small incisions under local anaesthetic. In the United States, approximately half of AAA repairs are by endovascular techniques [4].

\subsection{Redundant configurations}

When the inserted graft is too long for a given patient's anatomy, the redundant (extra) length adopts a convex configuration inside the aneurysm sac. This configuration is illustrated in Fig. 3.

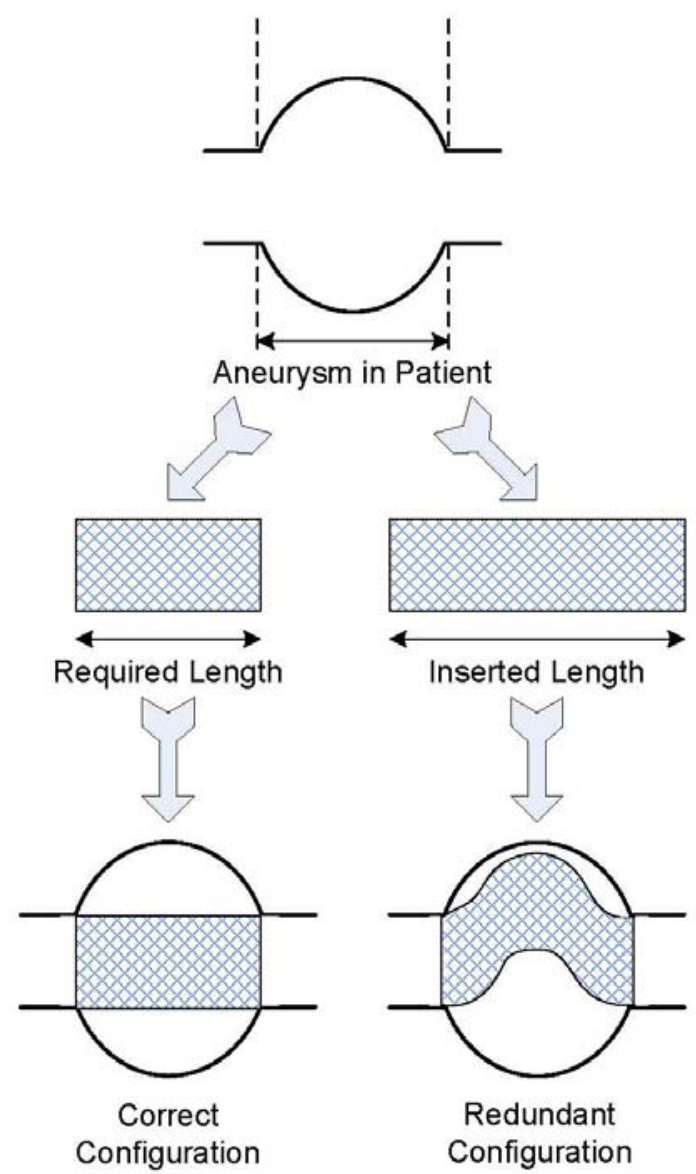

Figure 3: Schematic of graft placement inside aneurysm demonstrating the origin of graft redundancy. 
In both open vascular and endovascular repairs for aneurysms, avoidance of graft limb redundancy has been advocated in order to decrease the risk of limb kinking. However, modern commercial stent-grafts boast designs that prevent kinking despite sharp curvatures and redundancies. This allows more flexibility in stent-graft length discrepancies, as excessive length can be accommodated as a redundant segment in the aneurysm sac.

However, there is a clinical concern that this redundant configuration can lead to a significant increase in displacement force acting on the stent-graft devices. During open surgical repair, when a graft is redundant, the pressurized graft pushing out in a convex configuration gives the sense that the graft would dislodge if it were not for the sutures fixing it to the artery. Given that stent-grafts used in endovascular repair are not fixed by sutures like in open aneurysm repair, but rather, rely on other fixation methods like friction-fit, concerns about increased displacement forces are taken very seriously. Additionally, given that most stentgrafts are modular, increased displacement forces may also have an effect on the modular connections between device components. Figure 4 schematically shows three possible scenarios of stent-graft configuration inside an aneurysm sac: namely zero-, moderate- and severe-redundancy.
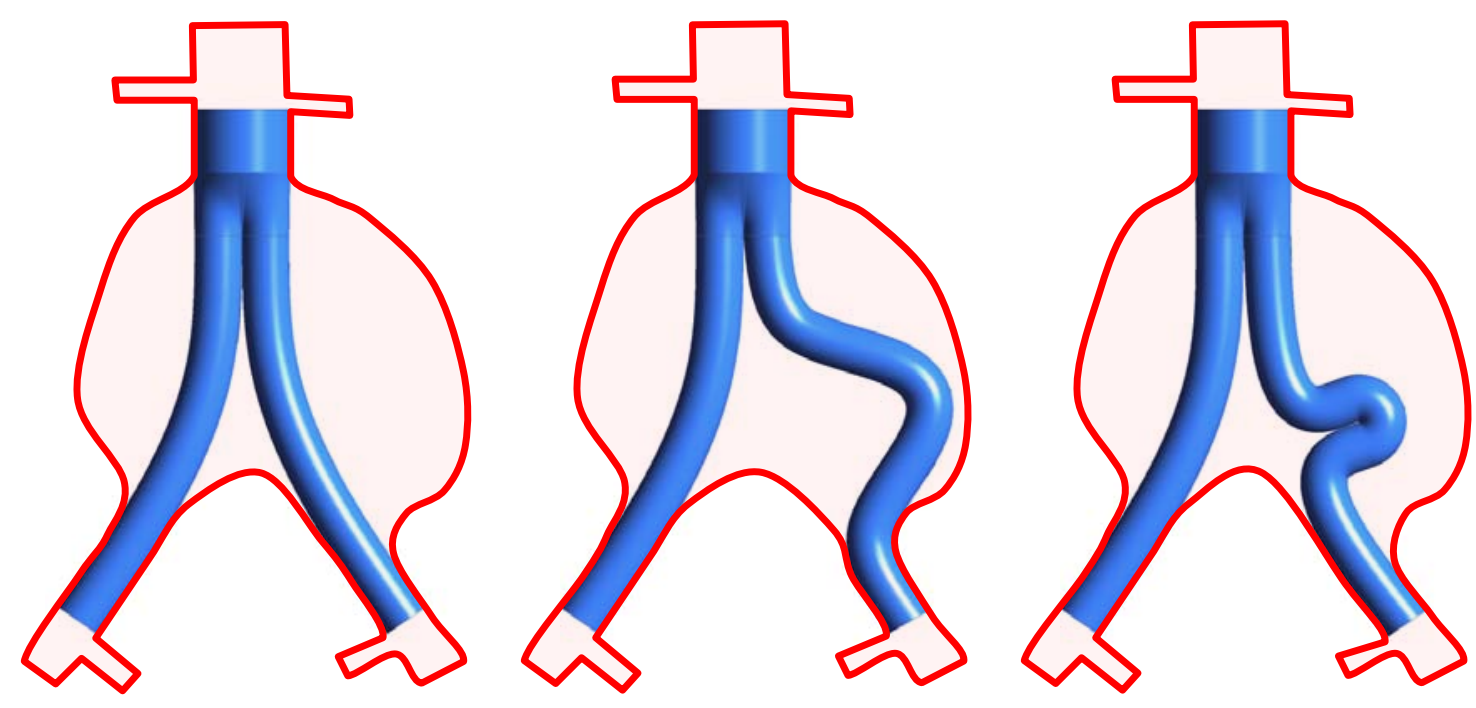

Figure 4: Drawings of examples of grafts with redundant limbs in aneurysm sac. 
There is presently no method to directly measure displacement force clinically in vivo, so this research estimates these forces using simulation techniques that are more readily available.

\subsection{Objective}

We hypothesize that redundant stent-graft configurations increase the downward force acting on the device, which may increase the risk of device dislodgement and failure. This can be clinically significant with serious implications. The present work analytically and numerically studies both steady-state and physiologic pulsatile blood flow through various redundant stent-graft configurations. An analytical approach is applied first to obtain a general approximation for the displacement forces acting on the device in steady-state conditions. Computational fluid dynamics (CFD) simulations are then used to predict values for displacement forces in different three-dimensional configurations with various degrees of redundancy. As the experimental measurements of stent-graft resistance to displacement have been performed in the downward direction, the numerical results of the present work are reported as the downward component of the force to make an appropriate comparison. This study will also estimate the effects of redundancy on the limb component dislocation force. Again, these results will be compared to reported values for component separation from experimental investigations. To our knowledge, there is no published study using CFD to investigate redundant endovascular stent-graft configurations. 


\section{Methods}

In this chapter, the methodologies of the analytical and computational approaches are discussed. The analytical approach is very inexpensive with respect to computational costs. The expensive computational simulations were run on an 8-processor computer $(3.0 \mathrm{GHz}$ Intel Xenon cores, each with 4 GB RAM for total of 32 GB of RAM) using the Linux SUSE (Novell, Waltham, MA) operating system.

\subsection{Analytical method}

An analytical approach was applied first to obtain a simplified, general approximation of displacement forces incurred by the flow of pressurized blood through various stent-graft geometries. Figure 5 is a schematic representation of the geometries and variables used to determine the displacement forces. Three simplified geometries of zero-redundancy (straightlimb), moderate-redundancy $\left(90^{\circ}\right.$-bend), and severe-redundancy $\left(180^{\circ}\right.$-bend $)$ were considered. These simplified geometries enabled us to use the available relations for the hydrodynamic head loss in pipe-bends. 

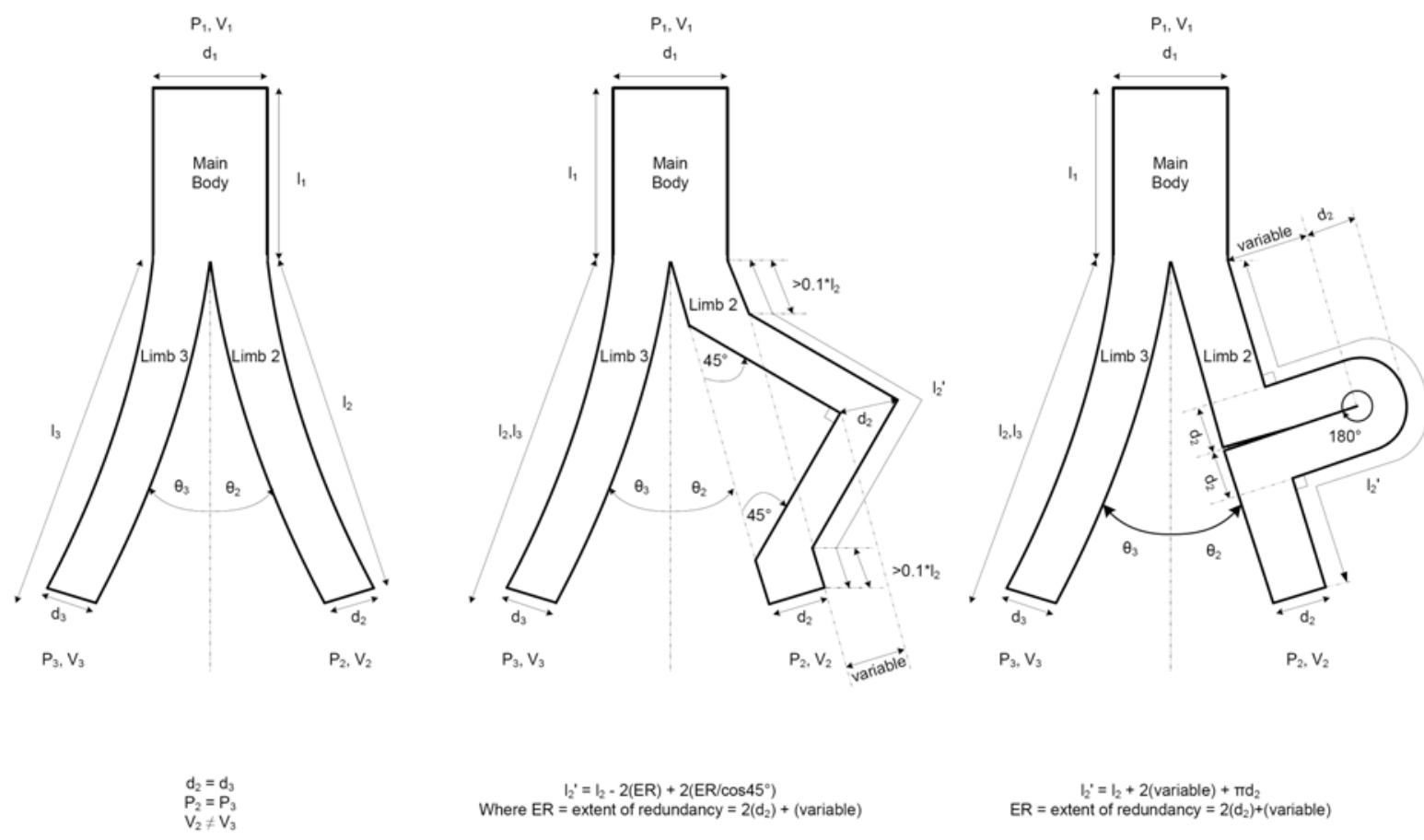

Figure 5: Schematic of zero-redundancy (straight-limb, on left), moderate redundancy ( $90^{\circ}$-bend, in middle), and severe-redundancy $\left(180^{\circ}\right.$-bend, on right) configurations used in the analytical approach.

The following equations were obtained by writing the pressure force and momentum balances to determine the displacement forces acting on the stent-graft in the horizontal (x) and vertical (y) directions (see Appendix I for details of force derivation):

$F_{x}=P_{3} A_{3} \sin \theta_{3}+\rho v_{3}^{2} A_{3} \sin \theta_{3}-P_{2} A_{2} \sin \theta_{2}-\rho v_{2}^{2} A_{2} \sin \theta_{2}$

$F_{y}=P_{1} A_{1}+\rho v_{1}^{2} A_{1}-P_{2} A_{2} \cos \theta_{2}-\rho v_{2}^{2} A_{2} \cos \theta_{2}-P_{3} A_{3} \cos \theta_{3}-\rho v_{3}^{2} A_{3} \cos \theta_{3}+F_{\text {weight } y}$ 
In the above equations, $v_{2}, v_{3}, \mathrm{P}_{2}$, and $\mathrm{P}_{3}$ are four unknowns that need to be determined for force prediction. The first auxiliary equation to predict these four unknowns was obtained by setting the outlet pressure of the two limbs equal, similar to the analytical works by Liffman et al. [5], Finol et al. [6], and Morris et al. [7]. While the latter two groups studied symmetric geometry, Liffman et al. included asymmetric geometries in their analytical work. Writing the mass balance between the inlet and the two outlets provides the second auxiliary equation. In order to determine the remaining two auxiliary equations, the modified Bernoulli energy equation was applied to each limb of the bifurcated flow path:

$$
\frac{P_{\text {out }}}{\rho g}+\alpha_{\text {out }} \frac{V_{\text {out }}^{2}}{2 g}+Z_{\text {out }}=\frac{P_{\text {in }}}{\rho g}+\alpha_{\text {in }} \frac{V_{\text {in }}^{2}}{2 g}+Z_{\text {in }}+h_{\text {loss }}
$$

The 'head loss' term in Eq. 3 includes both the friction loss and minor losses due to the graft bending. Building on the work of Liffman et al. [5], pipe roughness for the friction loss calculations and curved fittings for the minor loss calculations were considered. The different lengths of the graft limbs in the three geometries were accounted for in the friction loss calculations. The empirically-derived loss coefficients $(\xi)$ for curved fittings of $45^{\circ}, 90^{\circ}$, and $180^{\circ}$ were used to estimate the minor losses [8]. The total head loss was then calculated for each limb as follows:

$$
h_{\text {loss_ serial }}=f_{1}\left(\frac{v_{1}^{2}}{2 g}\right)\left(\frac{L_{1}}{D_{1}}\right)+\sum \xi_{1}\left(\frac{v_{1}^{2}}{2 g}\right)+. .+f_{n}\left(\frac{v_{n}^{2}}{2 g}\right)\left(\frac{L_{n}}{D_{n}}\right)+\sum \xi_{n}\left(\frac{v_{n}^{2}}{2 g}\right)
$$

The four auxiliary equations were then used to predict the velocity and pressure at the outlets of the two limbs using an iterative method. The predicted values of the velocity and pressure were then used in Eqs. 1 and 2 to predict the displacement forces acting on the stent-graft. 
The analytical model was created using Matlab (MathWorks, Natick, MA), and allowed us to vary the degree of limb redundancy. Additionally, the diameters, lengths, and angles of the stent-graft model as well as the velocity and pressure could be varied. The validity of the analytical model was confirmed by accurately reproducing results of other published work that had used analytical methods [5, 6] (see Appendix II).

\subsection{Computational fluid dynamics}

Analytical methods for prediction of stent-graft displacement forces require extreme simplification and significant assumptions [5-7, 9]. In order to accommodate threedimensional geometries with more realistic configurations, the computational fluid dynamics approach can be used to simulate the blood flow through the reconstructed stent-graft geometries.

Stent-grafts lend themselves well to CFD studies of blood flow behaviour. The ability to define the geometric flow channel is straightforward and the clinical utility is high. Early studies focused on numerical analysis of untreated aneurysms [10-13]. These allowed improved estimation of rupture risk for specific aneurysm shapes. With the rapid development and dissemination of EVAR, CFD has been applied to model the haemodynamics of stent-graft devices. One of the earliest and most cited numerical studies of stent-grafts is the work of Liffman et al. [5]. Since then, various authors have been adding more complexity to the CFD models. Howell et al. applied pulsatile flow to their numerical model [14]. Morris et al. have further characterized analytical studies of stent-graft flow by adding pulsatile flow and graft compliance [7]. Other studies have pursued patient-specific geometries $[14,15]$ and comparisons of pre- and post-EVAR scenarios [16-18]. In a recent review of biomechanical behavior and performance of stent-grafts, Corbett et al. noted that as the technique of EVAR matures, the focus of research is moving from feasibility studies to assessments of failure mechanisms [19]. CFD studies have been instrumental in identifying factors that cause increased displacement force that increases the risk of device migration. The results are generally described in terms of anchoring force required to fix the device in place. The factors affecting this force have been identified with the aid of CFD techniques, 
and include increasing proximal diameter, proximal neck angle, iliac angle, hypertension, and configurations that involve significant differences between inlet and outlet diameters [20, 21].

\subsection{Geometry creation}

Stent-graft dimensions used in published computational simulations vary widely, and there is no consistent or accepted standardized geometry. The following table summarizes the dimensions of different stent-graft geometries studied in the literature:

Table 1: Dimensions for stent-grafts used in CFD studies in the literature.

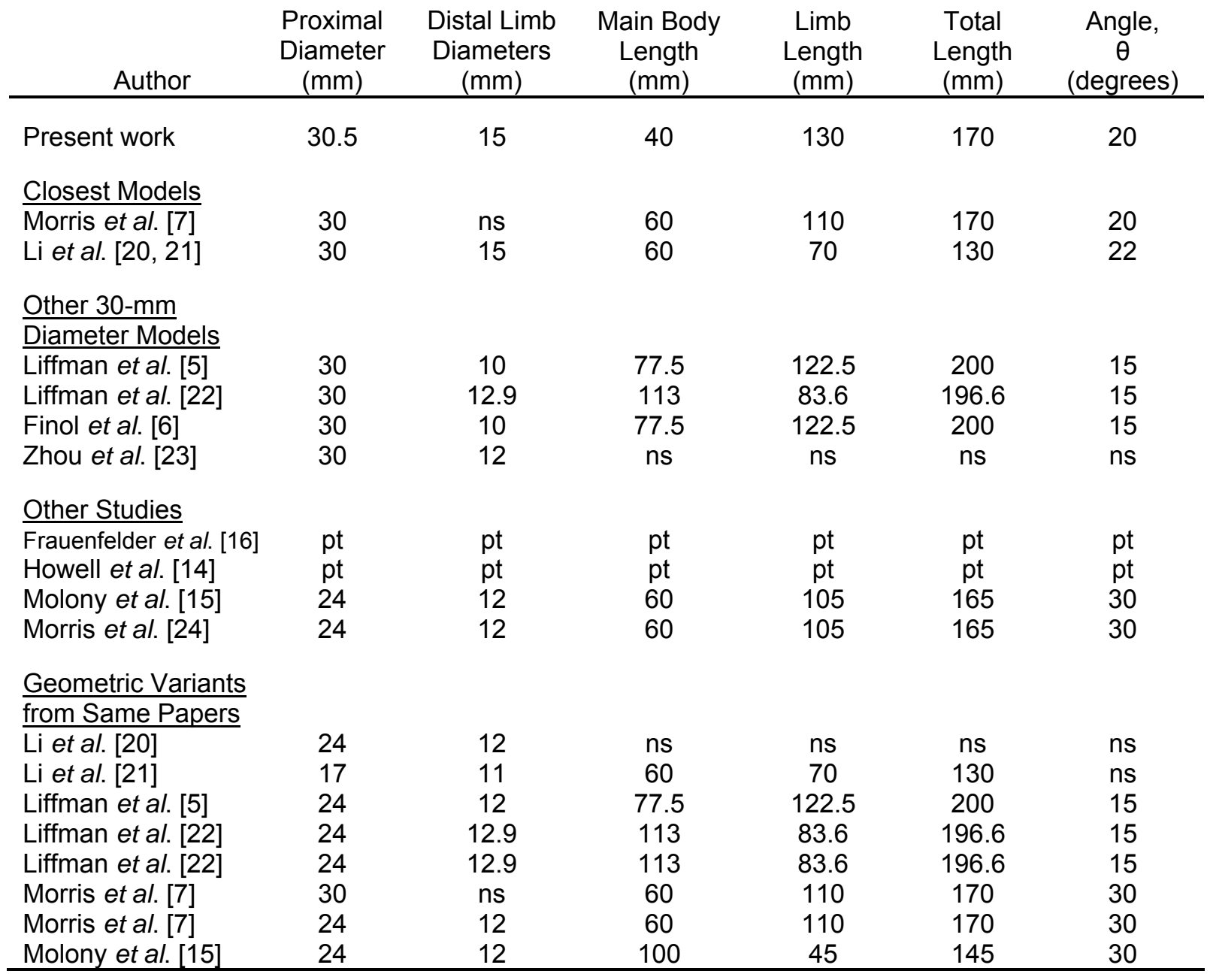

${ }^{*}$ ns $=$ not specified; $p t=$ patient specific 
We chose dimensions similar to a modern commercially-available device that easily accommodates redundant configurations. The Anaconda ${ }^{\mathrm{TM}}$ Endovascular Device (VascutekTerumo, Inchinnan, Renfrewshire, Scotland) is supported by multiple independent ring stents, which prevent kinking while offering tremendous flexibility.

Consistent with our analytical approach, three representative three-dimensional geometries were created for computational evaluation of zero-redundancy ('straight' limb), moderateredundancy $\left(90^{\circ}\right.$ convexity), and severe-redundancy ( $180^{\circ}$ convexity) configurations. These geometries are shown in Fig. 6:
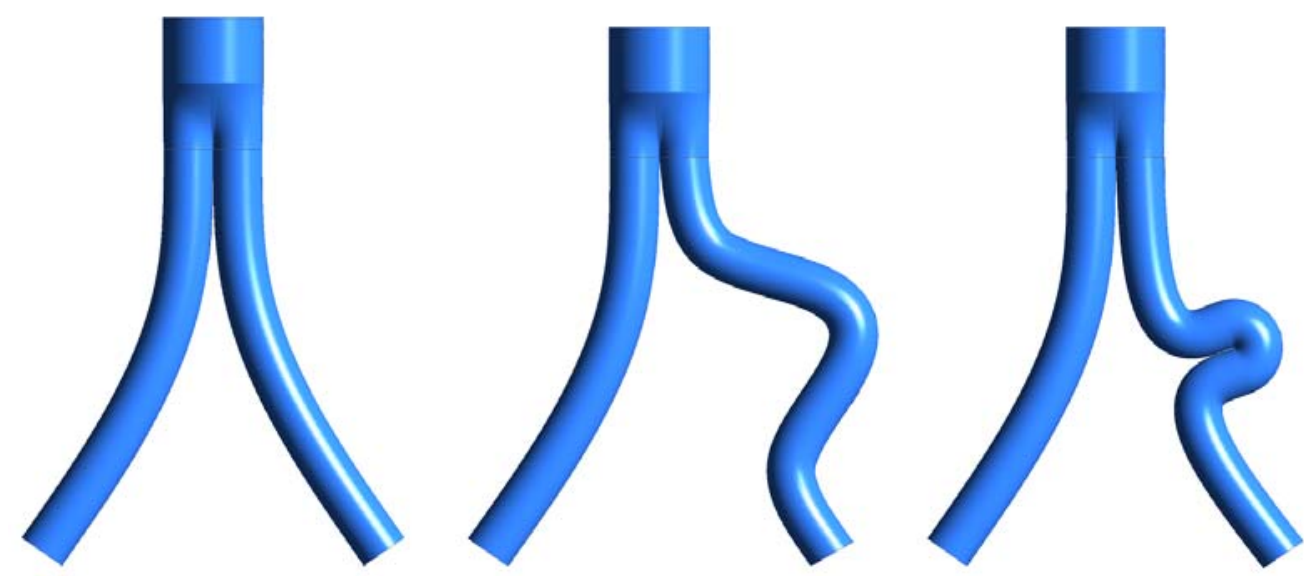

Figure 6 Three representative three-dimensional geometries created for numerical study of zero-redundancy (left), moderate-redundancy (middle), and severe-redundancy (right) configurations.

The geometries were constructed in SolidWorks (Dassault Systemes SolidWorks Corp., Concord, MA) based on a $30.5 \mathrm{~mm}$ diameter main body inlet with a length of $40 \mathrm{~mm}$ from the top of the graft to the flow divider. The 'straight' limbs were based on a diameter of 15 $\mathrm{mm}$ with a length of $130 \mathrm{~mm}$, to give a total device length of $170 \mathrm{~mm}$. The bifurcation angle used was $40^{\circ}$ (i.e., $20^{\circ}$ for each limb from the vertical midline). This is similar to the work of 
Morris et al. [7], Li et al. [21], and Liffman et al. [5] who used bifurcation angles of $40^{\circ}, 45^{\circ}$ and $30^{\circ}$, respectively. Clinical measurements by Sun et al. found the aortic bifurcation angles from patient MRI's to be in the range of $37.7^{\circ} \pm 10.2^{\circ}$ [25]. To be consistent with our analytical modeling, our redundant limbs used curved pipe angles of $45^{\circ}, 90^{\circ}$ and $180^{\circ}$. The redundant limbs maintained the same proximal and distal segments, but varied in the midportion to simulate the convexity from redundancy. The outlets of the three different geometries have the exact same area, orientation and angle as seen in Fig. 7; such that the observed difference in the predicted downward force solely relates to the redundancy.

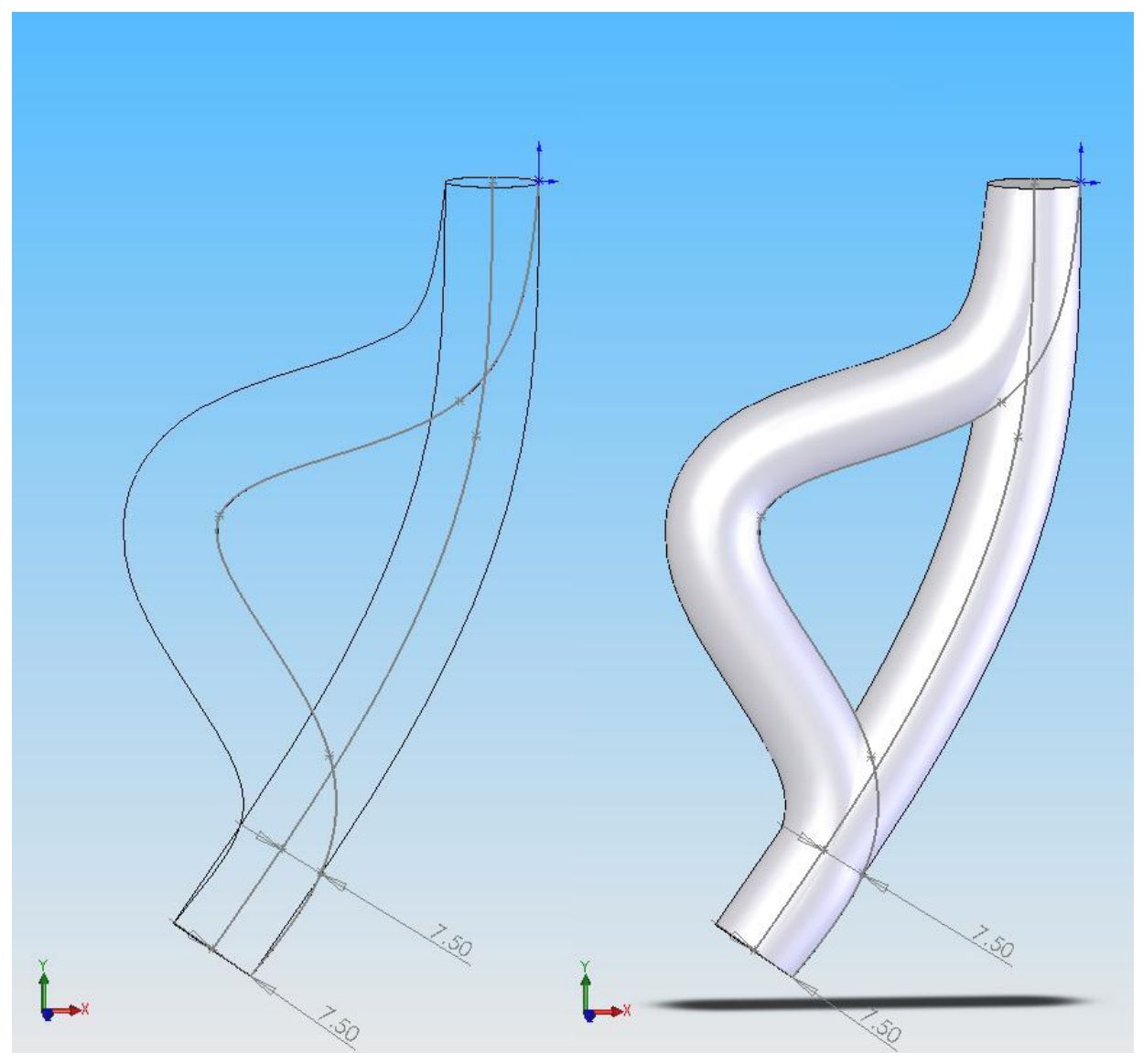

Figure 7: Creation of moderate-redundancy geometry with the same outlet angle and area as zero-redundancy geometry. 
This is important, as the magnitude of the pressure component, which is the dominant component of the total displacement force [14], is strongly affected by these parameters (see Appendix III for example). The redundancy was added to only one of the two limbs, so that the differences would be isolated to the redundancy in the single limb. Additionally, the geometry was oriented in a horizontal plane (patient lying down supine), so that the effects of gravity would be insignificant.

\subsection{Grid generation}

The geometries were then imported into the software Ansys ICEM CFD (ANSYS, Inc., Canonsburg, PA) to create the mesh. Although implementation of a hexahedral grid required more manual effort, we chose this over a tetrahedral grid because for our particular solver, it yielded more accurate results with a lower number of nodes. Examples of the tetrahedral and hexahedral meshes are shown in Figs. 8 and 9.

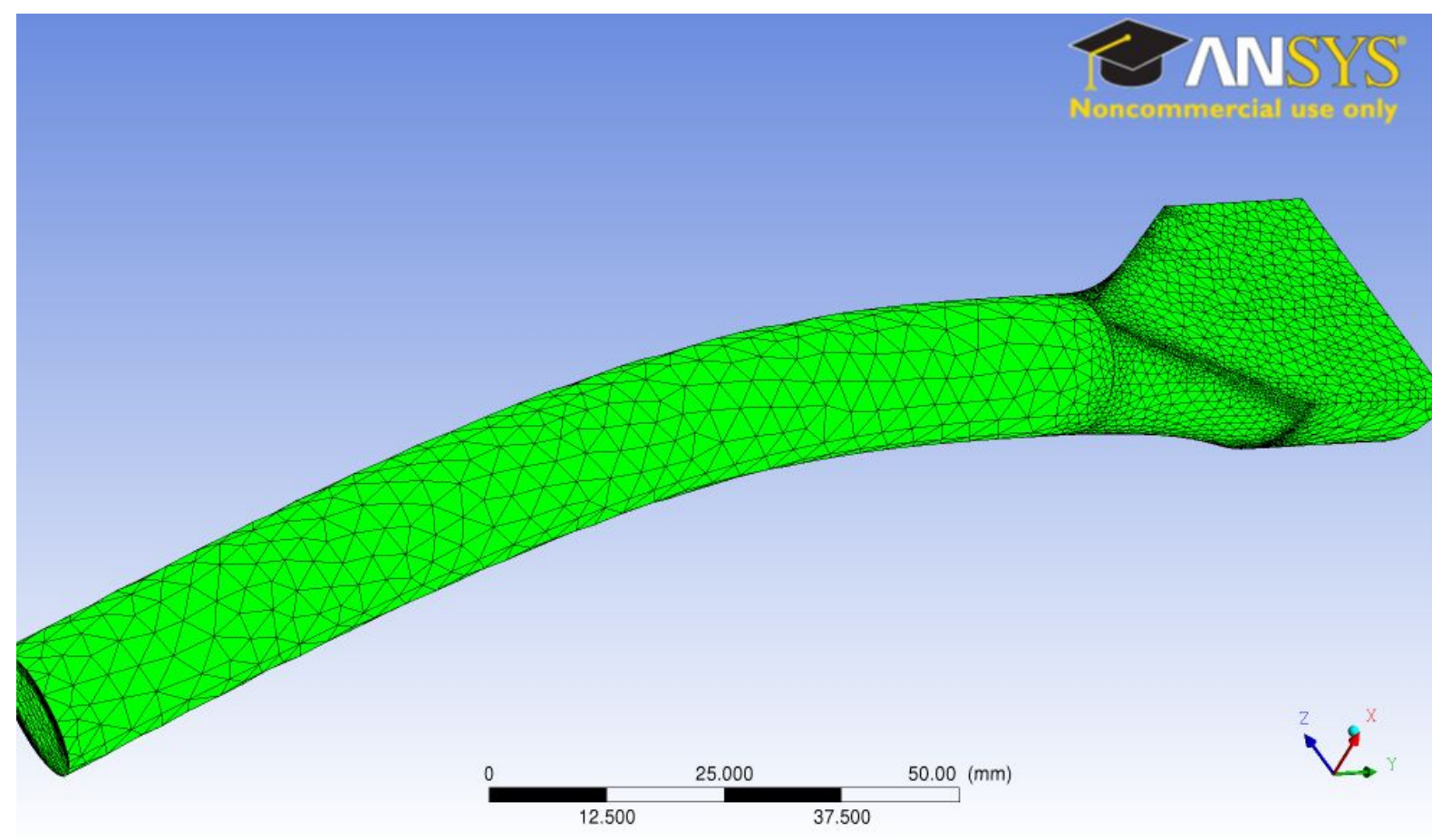

Figure 8: Example of tetrahedral meshing. Note that the surface is covered with triangles (one face of a tetrahedron). 

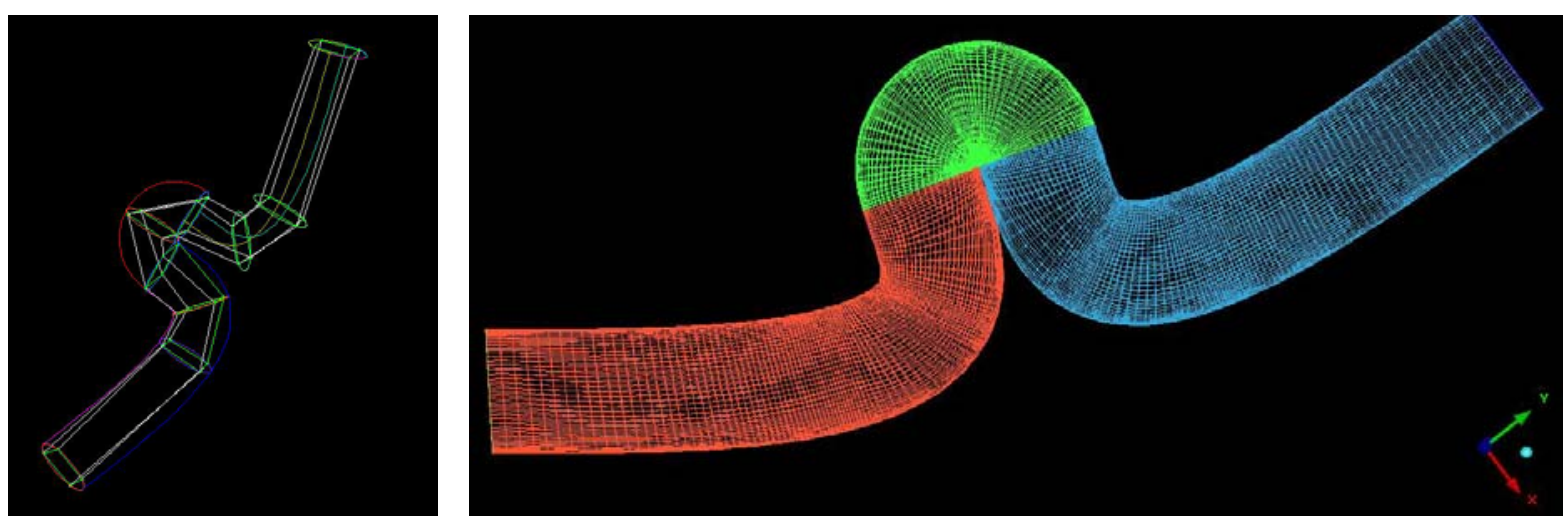

Figure 9: $\quad$ Example of blocking (left side) used to create hexahedral mesh (right side).

To evaluate the performance of hexahedral and tetrahedral elements on the solver used, the steady-state Poiseuille flow in a circular pipe was simulated using the two meshes with comparable numbers of nodes. As shown in Fig. 10, the hexahedral mesh can accurately predict the flow, while the tetrahedral mesh may require a finer mesh to obtain similar accuracy. We chose the hexahedral elements because of the smaller number of required elements. 


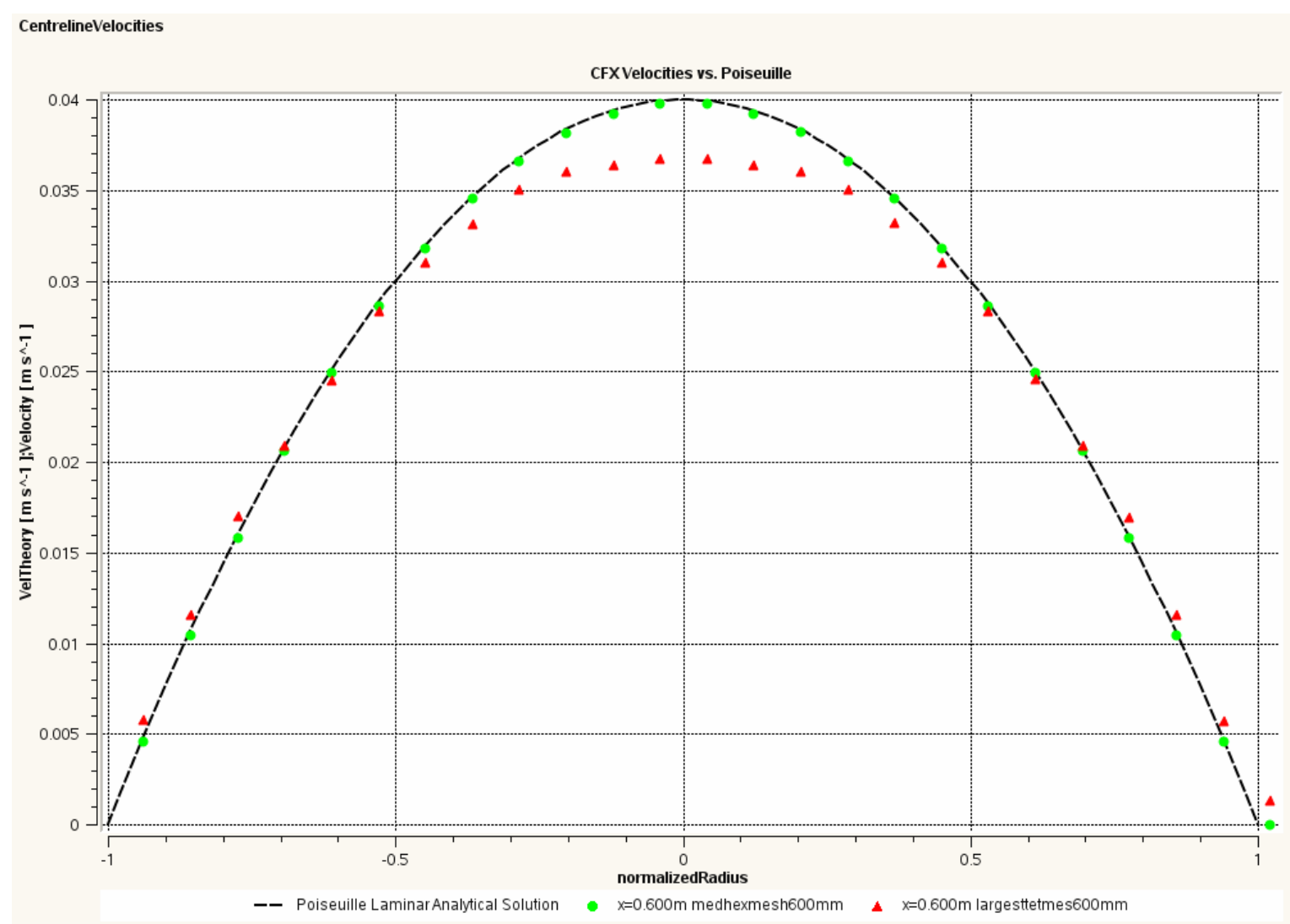

Figure 10: Plot of spatial velocity profile of a $600 \mathrm{~mm}$ straight pipe for analytical solution (dotted line), hexahedral mesh (green circles), and tetrahedral mesh (red triangles).

Grid refinement was applied for the near wall and high curvature areas. Figure 11 shows the outlet boundary face with the grid being finer near the walls and coarser near the centre, while still maintaining a hexahedral mesh. Figure 12 demonstrates the mid-plane $(\mathrm{z}=0)$ grid of the moderate-redundancy limb where the flow is from the right to the left. The grid is finer near the walls, at the curve, and slightly downstream from the curve. 


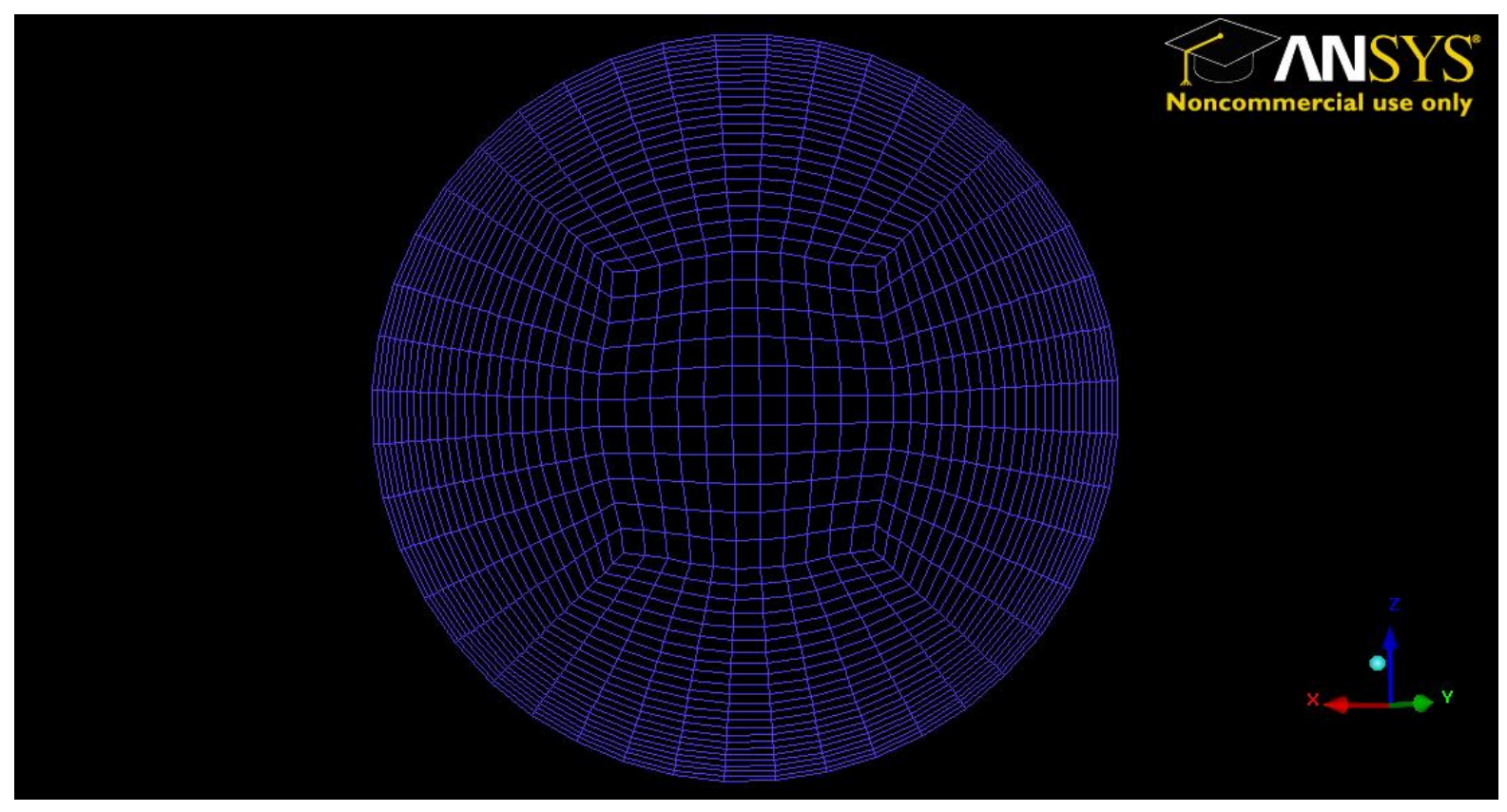

Figure 11: Example of outlet, demonstrating finer mesh near walls and coarser near centre, while maintaining a hexahedral mesh.

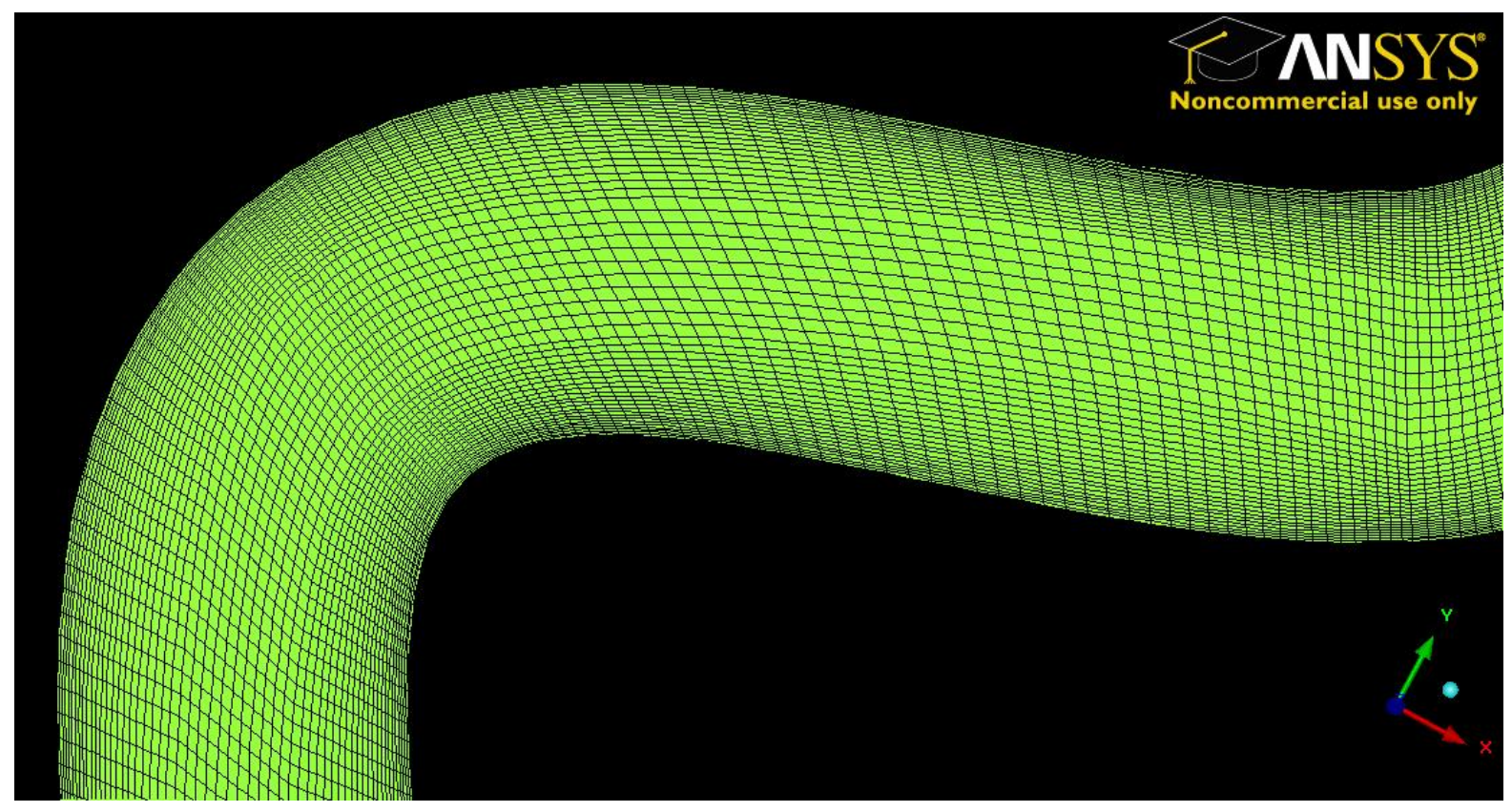

Figure 12: Example demonstrating the mid-plane grid of the moderate-redundancy limb where the flow is from the right to the left. The grid is finer near the walls, at the curve, and slightly downstream from the curve. 
In order to obtain mesh-independent results, multiple steady-state simulations were run for each geometry using different numbers of nodes. In the example shown in Fig. 13, the solution for downward force for severe-redundancy decreases by less than $1 \%$ between five different grid-sizes that we examined (the sixth, and largest, mesh was inconsistent with the other meshes with respect to force predictions as well as computational time). At the same time, the computational time increases significantly as shown in Fig. 14. We felt that the $1.9 \times 10^{6}$-node grid would be a reasonable compromise, as the grid could become extremely large without any addition to the clinical significance of the result if we continued to increase the grid size. Similar grid studies were performed for the zero- and moderate-redundancy configuration meshes. We chose meshes with sizes of $\sim 750,000, \sim 890,000$ and $\sim 1,900,000$ nodes for the zero-, moderate-, and severe-redundancy geometries, respectively.

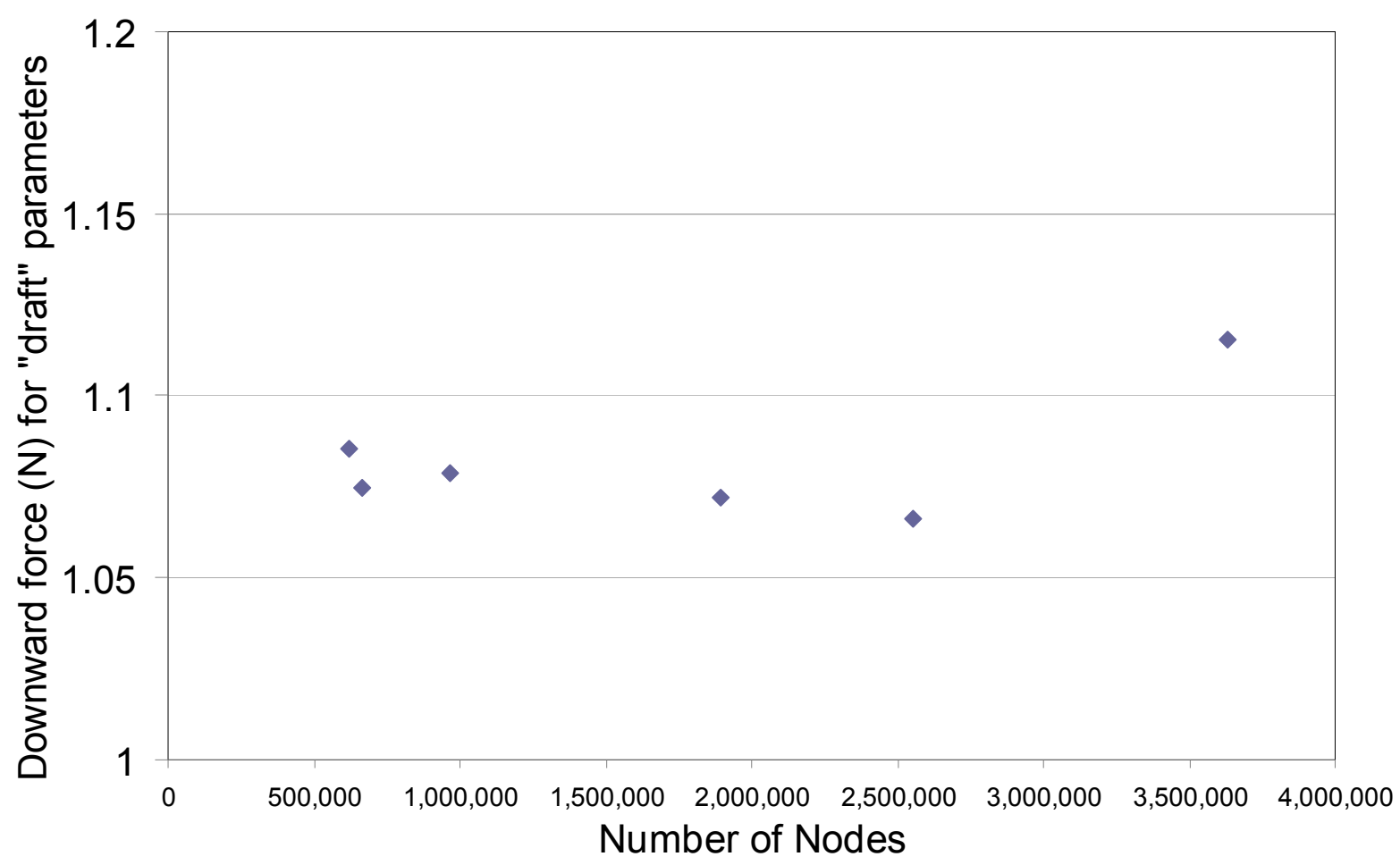

Figure 13: Plot of computationally predicted downward force (in Newtons) versus number of nodes for severe-redundancy grids. The $1.9 \times 10^{6}$-node grid was used. 


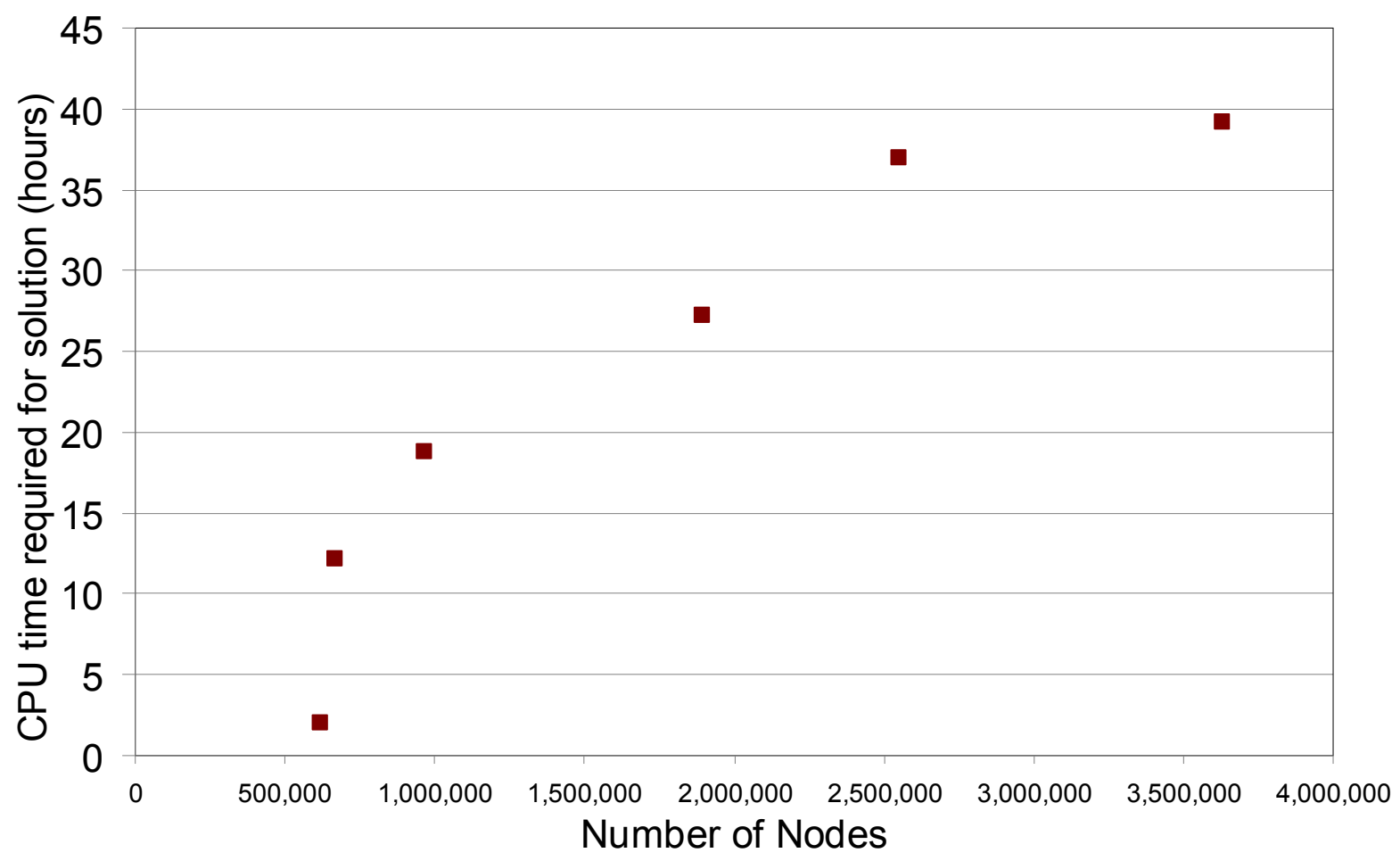

Figure 14: Plot of CPU time required for solution (in Hours) versus number of nodes for severe-redundancy grids. The $1.9 \times 10^{6}$-node grid was used.

\subsection{Physical properties of blood}

While blood is non-Newtonian when examined over a wide range of velocities, for the velocities encountered in the abdominal aorta the relationship of shear stress and shear rate is close to linear [7]. Therefore, similar to other works [7, 15, 16, 24], we modeled blood as a Newtonian fluid with a constant dynamic viscosity of $0.0035 \mathrm{~Pa} \cdot \mathrm{s}$. As in other published works $[6,7,15,20,21,24]$, blood was treated as an incompressible fluid with a density of $1050 \mathrm{~kg} / \mathrm{m}^{3}$. 


\subsection{Boundary conditions}

The no-slip condition was set at the walls. The walls were considered rigid, since the compliance of the fabric walls is significantly less than native vascular tissue [7] .

For the steady-state simulations, the inlet velocity was set equal to $0.53 \mathrm{~m} / \mathrm{s}$, which is the peak systolic velocity for an experimentally-measured physiologic velocity profile [7]. While the outlet boundary condition for most CFD studies on EVAR has been a specified pressure, most have been studying symmetric geometries whereby the outlet pressures can be set equally [7, 20-22]. However, the equal outlet pressure condition has also been used in the studies with asymmetric limb configurations [5, 15, 24]. The commercial software Ansys CFX [26] specifically suggests that specified inlet-velocity and specified outlet-pressure is the most robust condition; with the second most robust condition being specified inletvelocity and specified outlet-mass flow. However, the equal outlet pressure condition does not reflect the condition that would be expected to occur naturally in vivo. The true condition would likely be found somewhere between the equal-pressure condition and the equal-mass flow condition (discussed further in section 4.5). We performed our studies using both equalpressure and equal-mass flow outlet conditions. For the first set of simulations, the outlet boundary condition was set as constant pressure of $16 \mathrm{kPa}(\sim 120 \mathrm{mmHg})$ for both limbs to simulate normal blood pressure conditions [27]. For the equal-mass flow condition, the mass flow for each of the two outlets was set at half of the inlet mass flow.

For transient simulations, we implemented an experimentally-measured time-dependent physiologic velocity profile at the inlet. Morris et al. approximated the pulsatile profile as a Fourier series [7]; the same series was used in this work. The coefficients for this Fourier series were supplied by T. McGloughlin through direct correspondence [28]. A plot of this profile is shown in Fig. 15. 


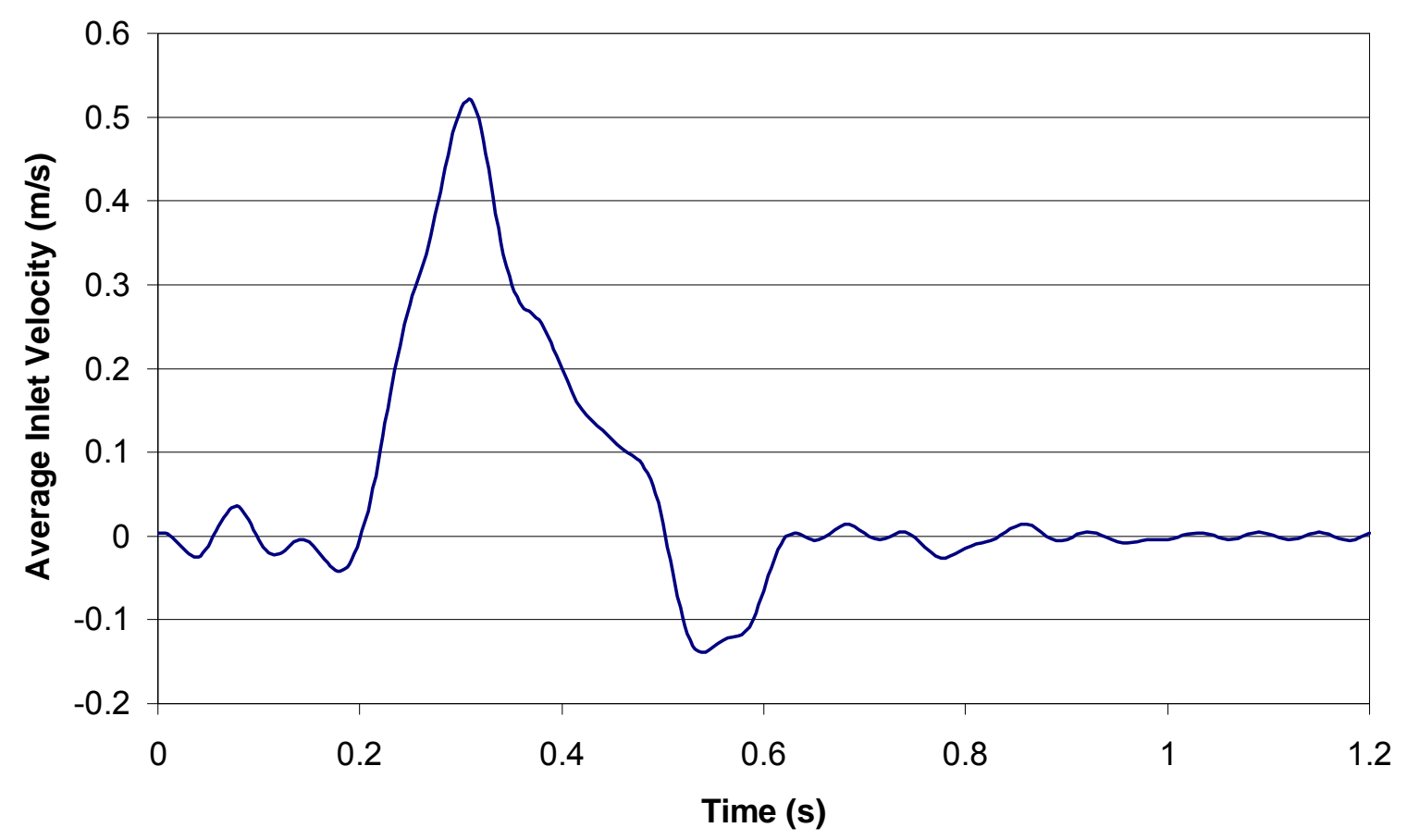

Figure 15: Plot of physiologic velocity profile (temporal) used for pulsatile studies. The coefficients for this Fourier series were supplied by T. McGloughlin through direct correspondence [28] and were those used in his previously published study [7].

The series, however, gives the spatially-averaged velocity across the inlet face as a function of time. However, the velocity profile with respect to space across the inlet face was implemented with a paraboloid profile. Examples of the three-dimensional velocity profile at the inlet of the solution domain are shown in Fig. 16 for the systolic and diastolic time phases. Note that during the time period from 0.51 seconds to 0.66 seconds, the inlet velocity is negative and is outward. 

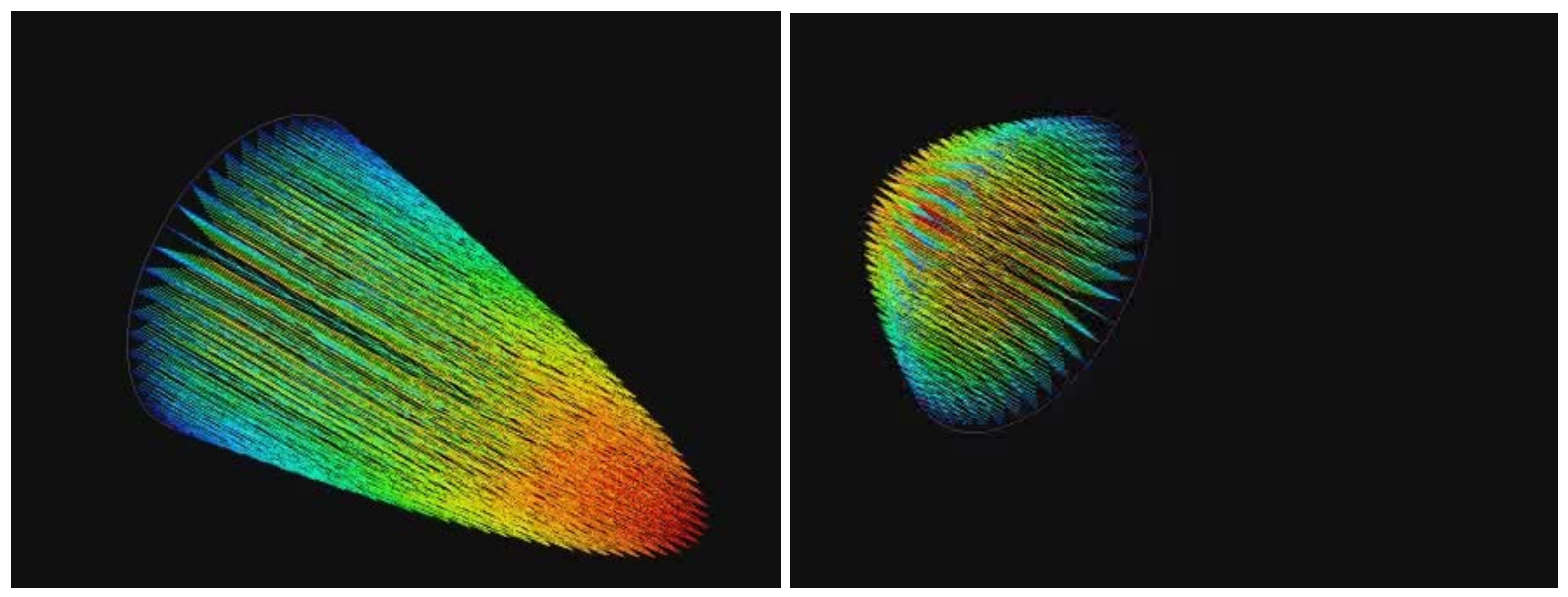

Figure 16: Examples of three-dimensional velocity profiles at inlet during forward-flow systolic phase (left) and reversed-flow diastolic phase (right).

Morris et al. have demonstrated that there is no significant difference in the predicted results when parabolic, flat, and physiologic-distributed spatial velocity profiles are used [24]. The outlet boundary condition was set as a physiologic pressure profile with respect to time for both limbs. The pressure profile at the outlet with respect to space was a flat profile, as the radial pressure gradient across the outlet surface is negligible.

\subsection{Steady-state flow solver}

The meshes for the main body and the various combinations of redundant limbs were put together in the software Ansys CFX-Pre, and the boundary conditions and solver parameters were applied to the merged mesh. The commercial software Ansys CFX 12.0 was used to perform the simulations. For steady-state flow, the average inlet velocity magnitude was set to the maximum systolic velocity, shown in Fig. 15. The inlet Reynolds number was calculated to be approximately 4700 based on the average velocity (in space) and the inlet diameter. This Reynolds number falls in the turbulent flow regime. Therefore, we used the low-intensity turbulence Shear Stress Transport (SST) model available in Ansys CFX 12.0, which is a combination of $\mathrm{k}-\varepsilon$ and $\mathrm{k}-\omega$ turbulence models [29]. 


\subsection{Pulsatile flow solver}

The software Ansys CFX 12.0 was chosen for the transient studies because of ease of implementation of the pulsatile velocity profile. The entire solution domain was initialized to zero-velocity and a pressure of $16 \mathrm{kPa}$. It was observed that the acceleration phase of systole dominated the profile at the start of the cycle, so that the results obtained for the peak force using one cycle and two cycles were identical (see results section 3.4). We therefore used only one cycle for the rest of the transient simulations reported. For the pulsatile study, although the Reynolds number becomes temporarily larger than 2300, a laminar solver for the simulations was used. As explained by Morris et al. [7], and Chatzimavroudis [30], acceleration during systole is felt to stabilize flow. The deceleration period after the peak velocity, which can be destabilizing, is very short, and flow quickly reaches lower Reynolds numbers in the laminar regime. The maximum displacement force for our studies occurs at peak systolic velocity; therefore, systole would be the most important segment of the cardiac cycle to be captured accurately.

\subsection{Convergence criteria}

For the steady-state flow simulations, convergence root mean square (RMS) of residuals for momentum and pressure (continuity) equations were set to $10^{-5}$ and $10^{-6}$ respectively. For the transient simulations, these values were less than $10^{-4}$ and $10^{-5}$ for each cycle, respectively. These are non-dimensional values that are normalized.

\subsection{Post processing}

The simulation results were processed in the commercial software Ansys CFX-Post. All simulations were checked to ensure that the inlet velocity profiles (temporal and spatial) were 
correct. The pressures and mass flows at the outlet boundaries were checked to confirm that the prescribed conditions were met.

The total acting force on all the walls was calculated for the $\mathrm{x}-, \mathrm{y}-$, and $\mathrm{z}$-directions. The $\mathrm{y}$ direction component was reported as the downward displacement force. For the limb component separation force, the force on the walls of only the redundant limb was calculated. Streamlines were visualized for all steady-state simulations. Velocity vectors were sampled on the mid-coronal $(\mathrm{z}=0)$ plane of the geometries for the transient (pulsatile) simulations at relevant time-points. 


\section{Results}

\subsection{Analytical steady-state flow}

The boundary conditions that were specified for the computational approach were applied to the developed analytical model. The results indicate an increase in the downward force as the redundancy of the stent-graft increases. The predicted downward displacement force for the zero-, moderate-, and severe-redundancy configurations is $6.38,6.48$ and $6.52 \mathrm{~N}$, respectively.

\subsection{Computational steady-state flow}

The simulated computational steady-state flow, consistent with analytical predictions, also predicts increasing displacement forces with increasing redundancy. The magnitude of the predicted total displacement forces for the zero-, moderate-, and severe-redundancy configurations with equal outlet mass flow are 7.36, 7.44 and $7.81 \mathrm{~N}$, respectively. The magnitude of the predicted total displacement forces for the zero-, moderate-, and severeredundancy configurations with equal outlet pressures are 7.49, 7.65 and $8.04 \mathrm{~N}$, respectively. The trend of increasing displacement force with increasing redundancy is clearly demonstrated in Fig. 17. 


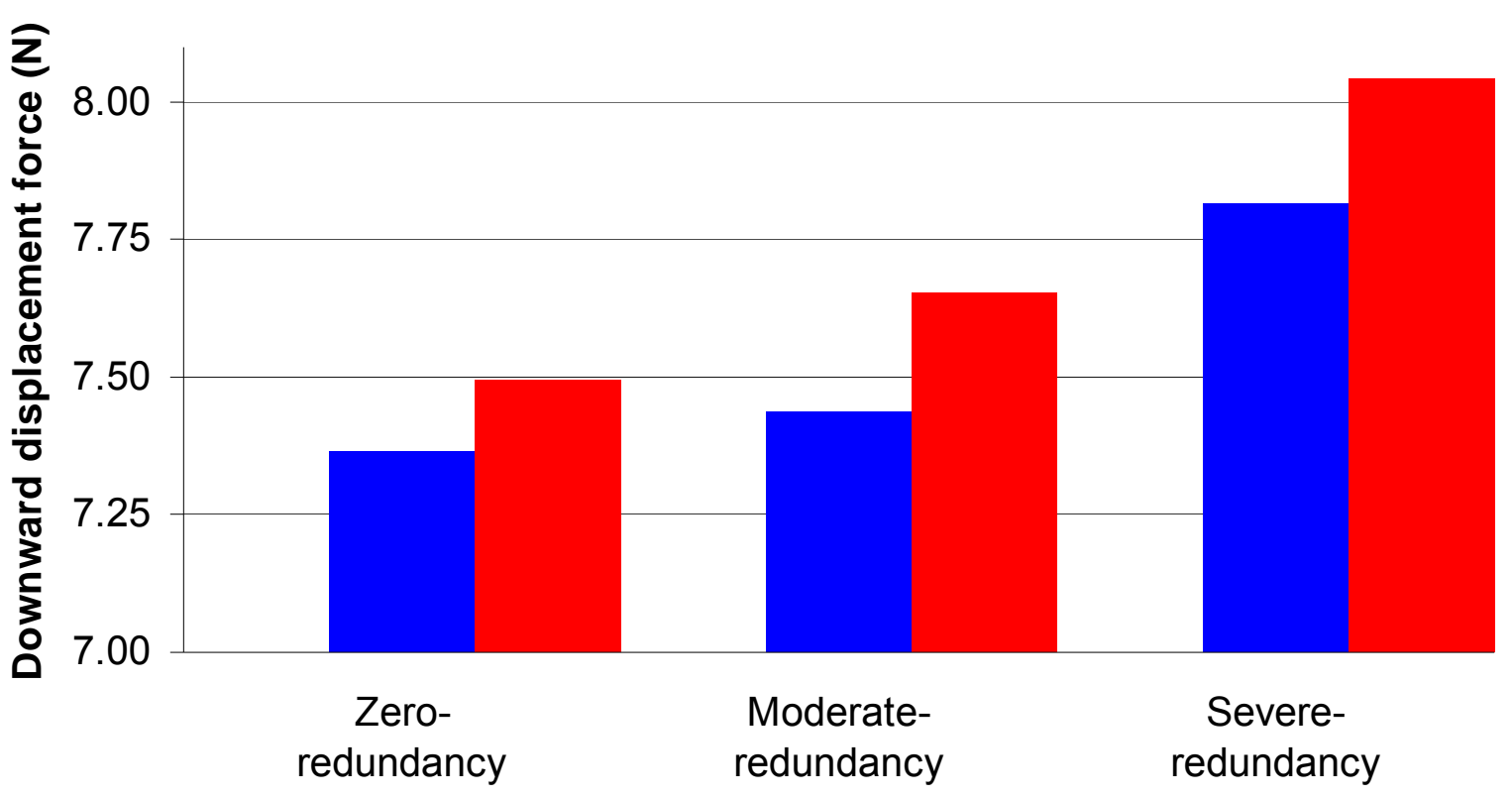

Figure 17: Downward displacement force for steady-state computational study for equalmass flow (blue) and equal-pressure (red) outlet conditions.

The downward components of the predicted displacement forces are essentially the same as the overall magnitude; indicating that for our geometries, the downward component is orders of magnitude larger than the forces in alternate directions and is the major constituent of the total forces. The lateral $\mathrm{x}$-direction components are only $6 \times 10^{-6}, 0.08$ and $0.2 \mathrm{~N}$ for the zero-, moderate-, and severe-redundancy configurations, respectively. As expected for our planar geometries, the out-of-plane z-direction components are negligible due to the symmetry of the domain in the z-direction.

An interesting finding during post-processing of the equal-pressure study is the degree of difference in mass flow between the two outlets. The ratio of mass flow between the two outlets for the zero-, moderate-, and severe-redundancy configurations is 50:50, 56:44, and 68:32, respectively. This suggests that given the equal outlet pressures and the increased head losses of the redundant limb, blood is preferentially flowing into the other limb. This will be reviewed further in the discussion (Section 4.5). 
As expected, the zero-redundancy configurations seen in Figs. 18 and 19 demonstrate symmetry; and there is no noticeable difference between the equal outlet mass flow and equal outlet pressure conditions. However, one can see differences in the distribution of flow between the equal-mass flow and equal-pressure models in the Figs. 20-23. These figures demonstrate more flow field streamlines directed down the limbs on the left when the equalpressure condition is applied, but equivalent down both sides for the equal-mass flow condition. On the other hand, one can see that the magnitudes of velocities are greater in the redundant limbs for the equal-mass flow condition.

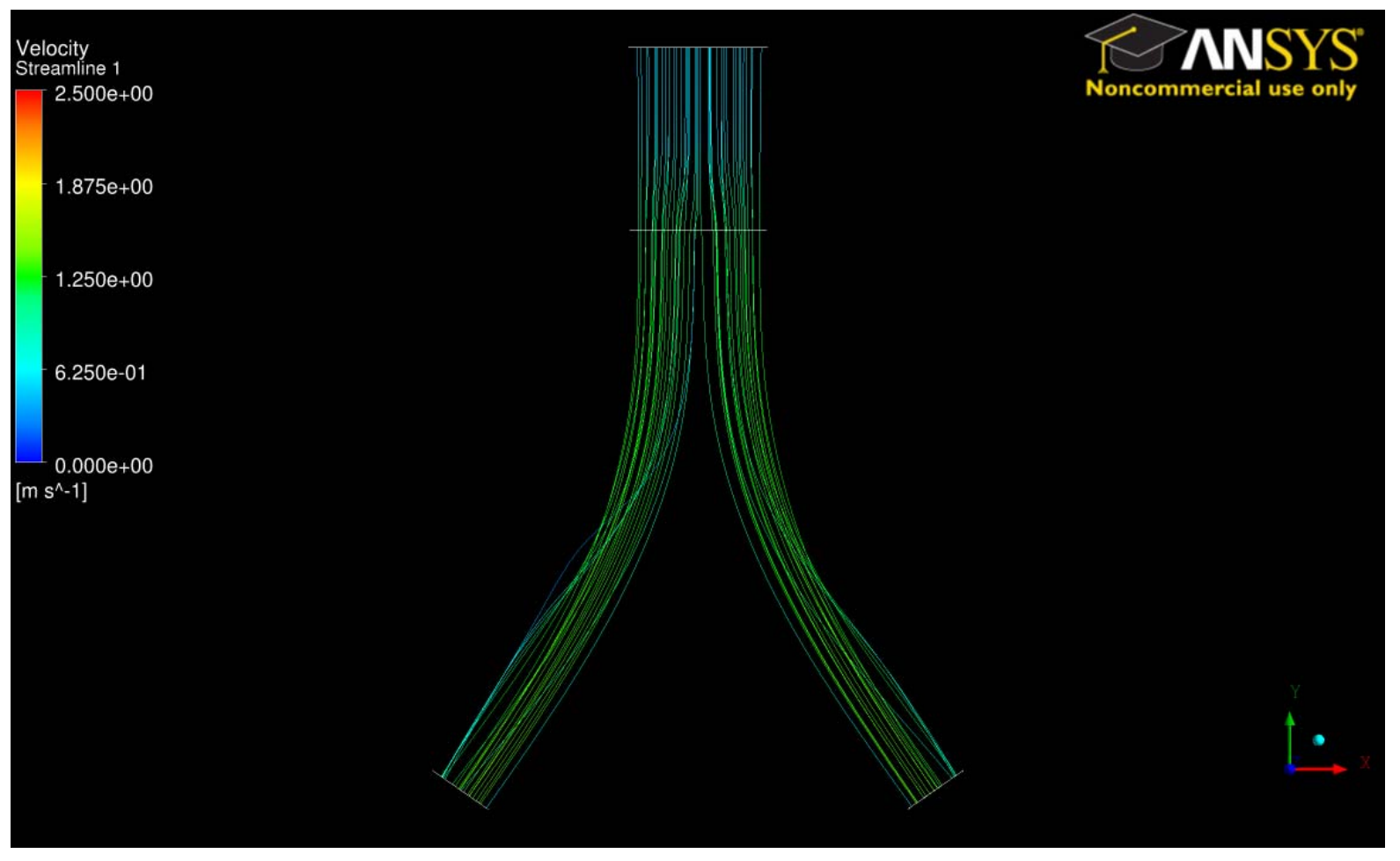

Figure 18: Equal outlet mass flow streamlines for zero-redundancy configuration. 


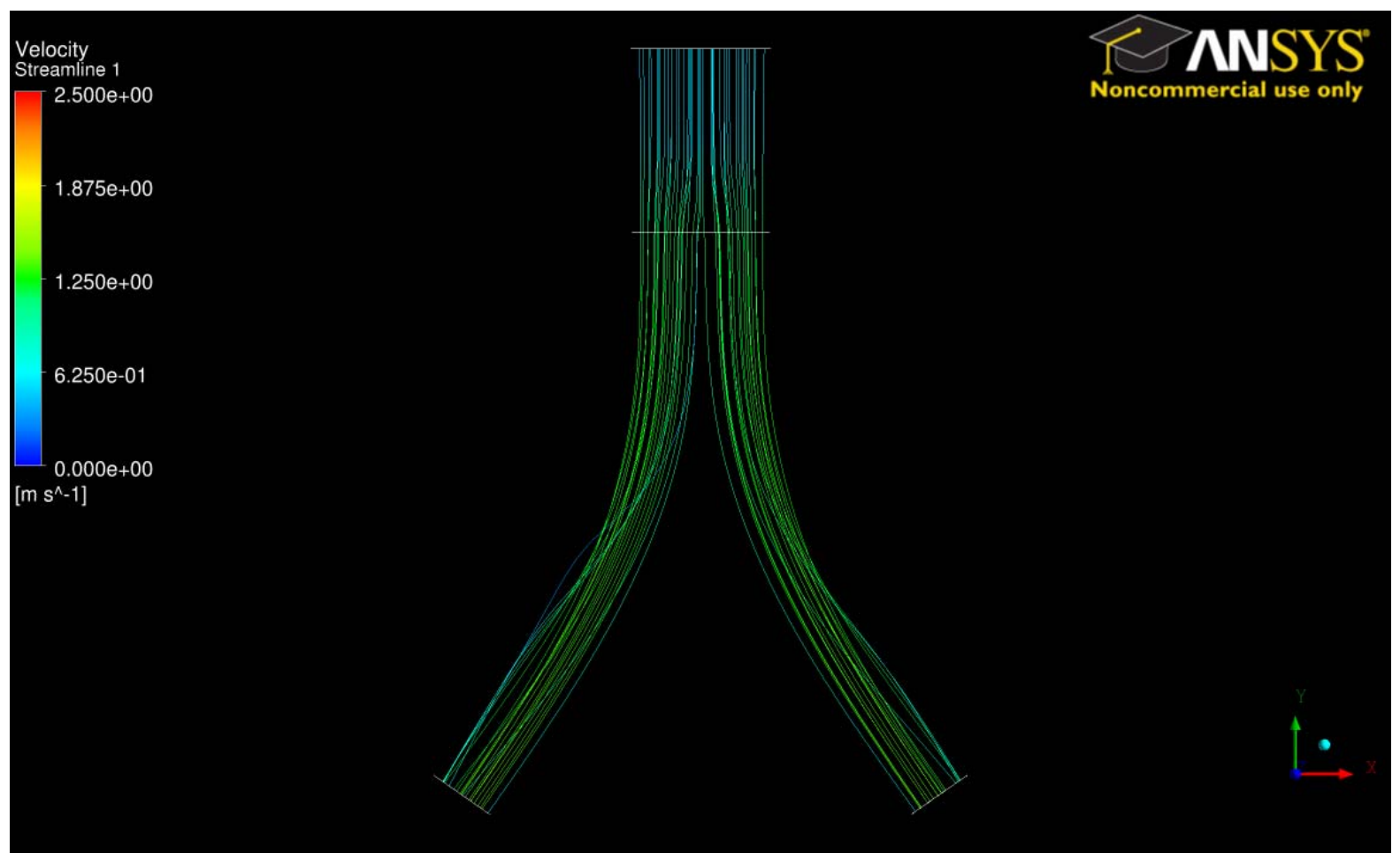

Figure 19: Equal outlet pressure streamlines for zero-redundancy configuration.

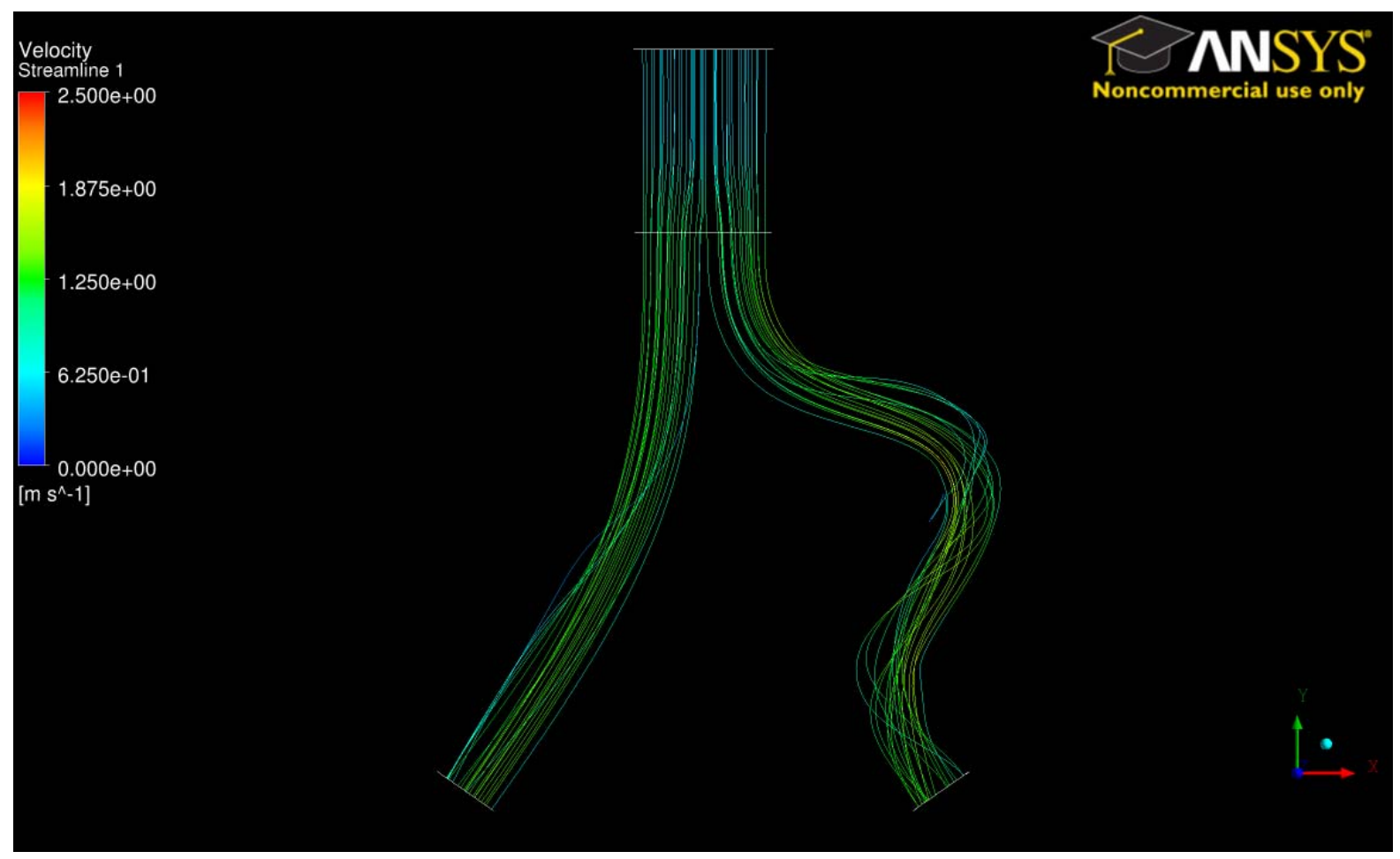

Figure 20: Equal outlet mass flow streamlines for moderate-redundancy configuration. 


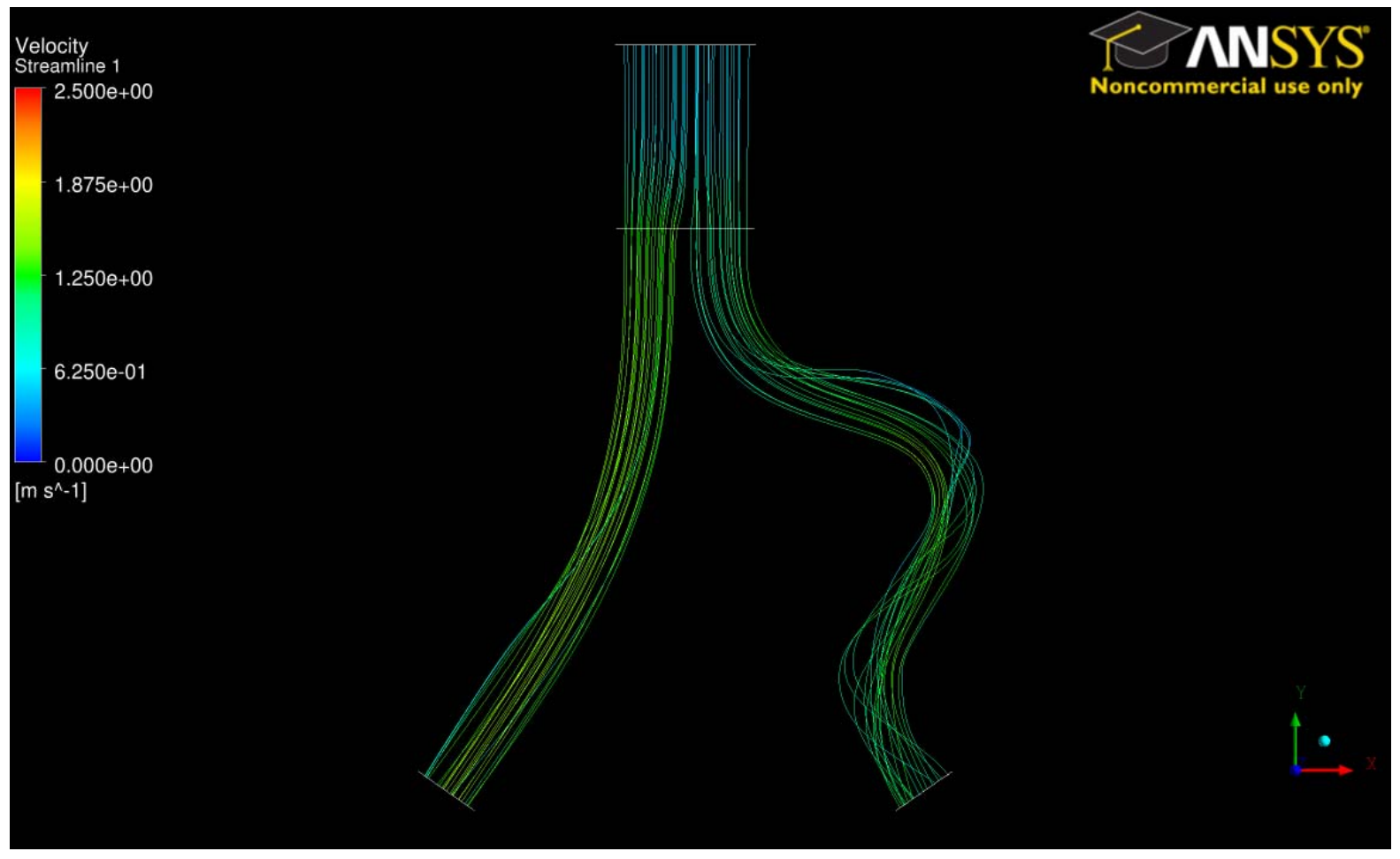

Figure 21: Equal outlet pressure streamlines for moderate-redundancy configuration. Note there are slightly more streamlines being "diverted" to the left limb on the left.

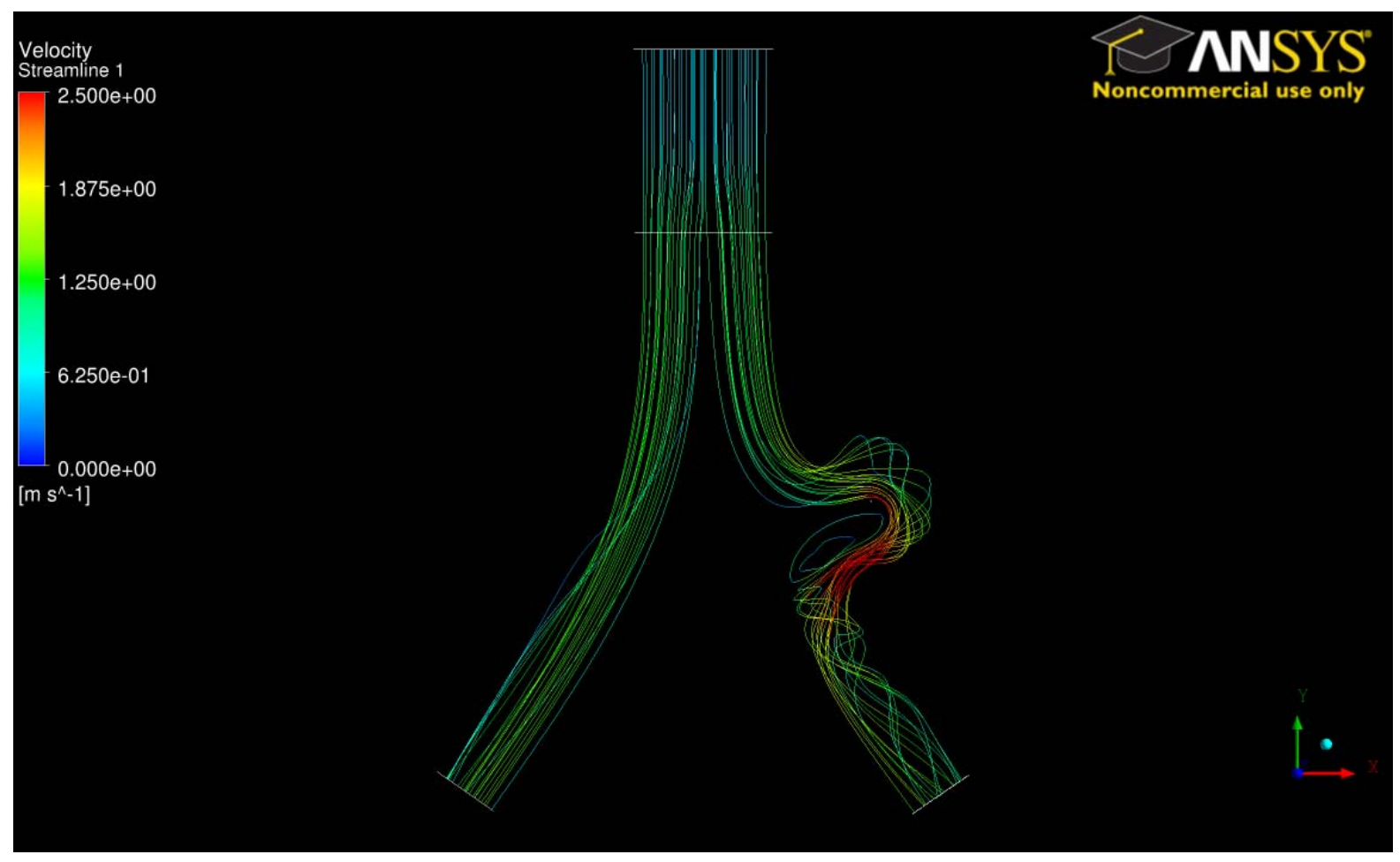

Figure 22: Equal outlet mass flow streamlines for severe-redundancy configuration. Note the increased velocities (indicated by red colour) for the limb on the right. 


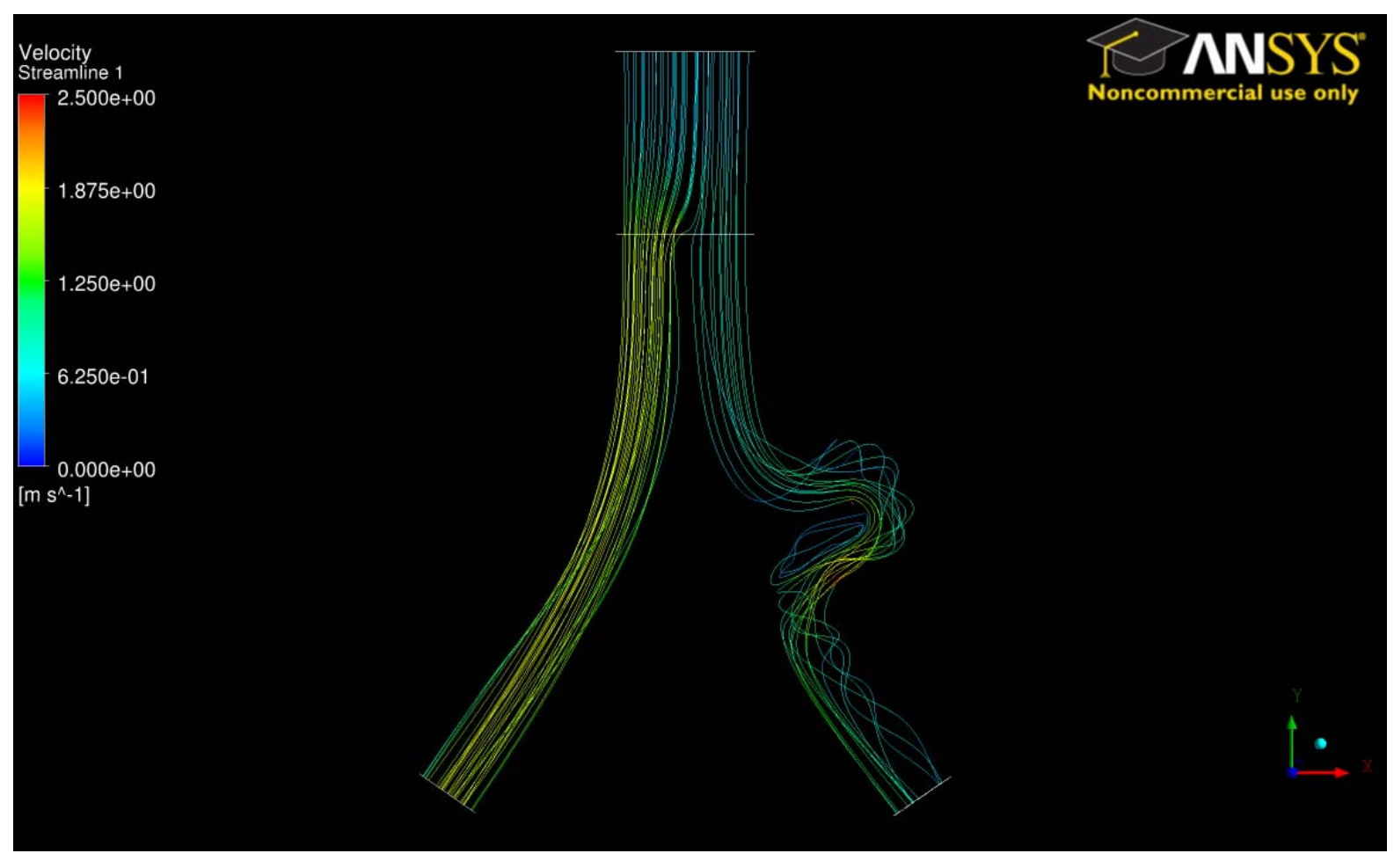

Figure 23: Equal outlet pressure streamlines for severe-redundancy configuration. Note there are more streamlines being "diverted" to the limb on the left.

The flow field streamlines in Figs. 22 and 23 demonstrate recirculation zones downstream of the sharp bends in the severe-redundancy configuration. It is important to note that there are no recirculation zones near the outlet boundaries to adversely affect the solution and the recirculation zone is limited to a small region after the bend.

The velocity vectors on the coronal $(\mathrm{z}=0)$ plane are visualized in Figs. 24-26. Similar to the flow field streamlines, there is symmetry for the zero-redundancy configuration; there are recirculation zones beyond the bend of the severe-redundancy configuration; and the velocity magnitudes are greater for the equal outlet mass flow condition. 


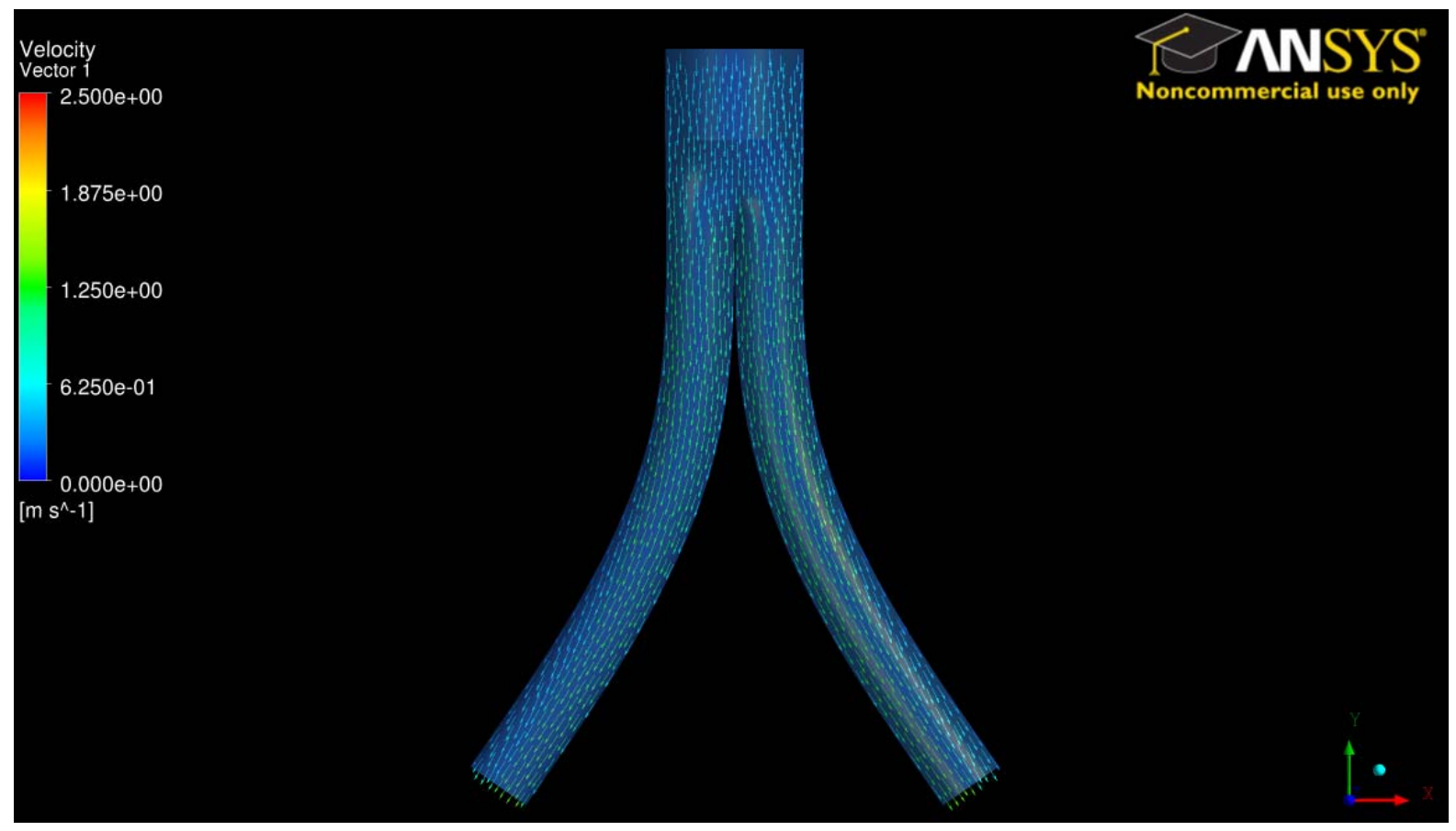

Figure 24: Velocity vectors on mid-coronal $(z=0)$ plane for zero-redundancy configuration under equal-pressure condition.

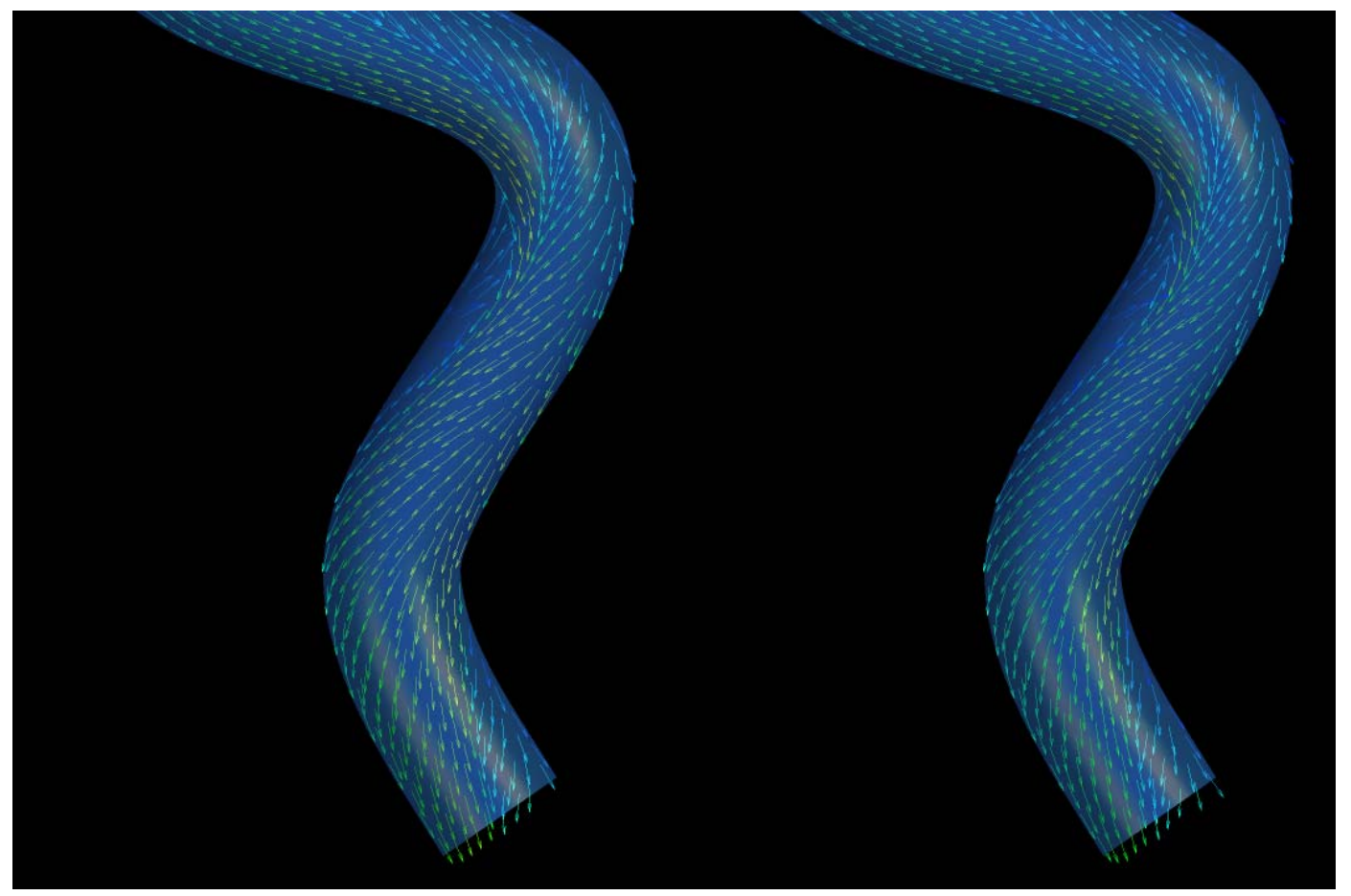

Figure 25: Velocity vectors on mid-coronal $(\mathrm{z}=0)$ plane for moderate-redundancy configuration under equal-mass flow (left) and equal-pressure (right) conditions. No recirculation zones near outlet boundary. 


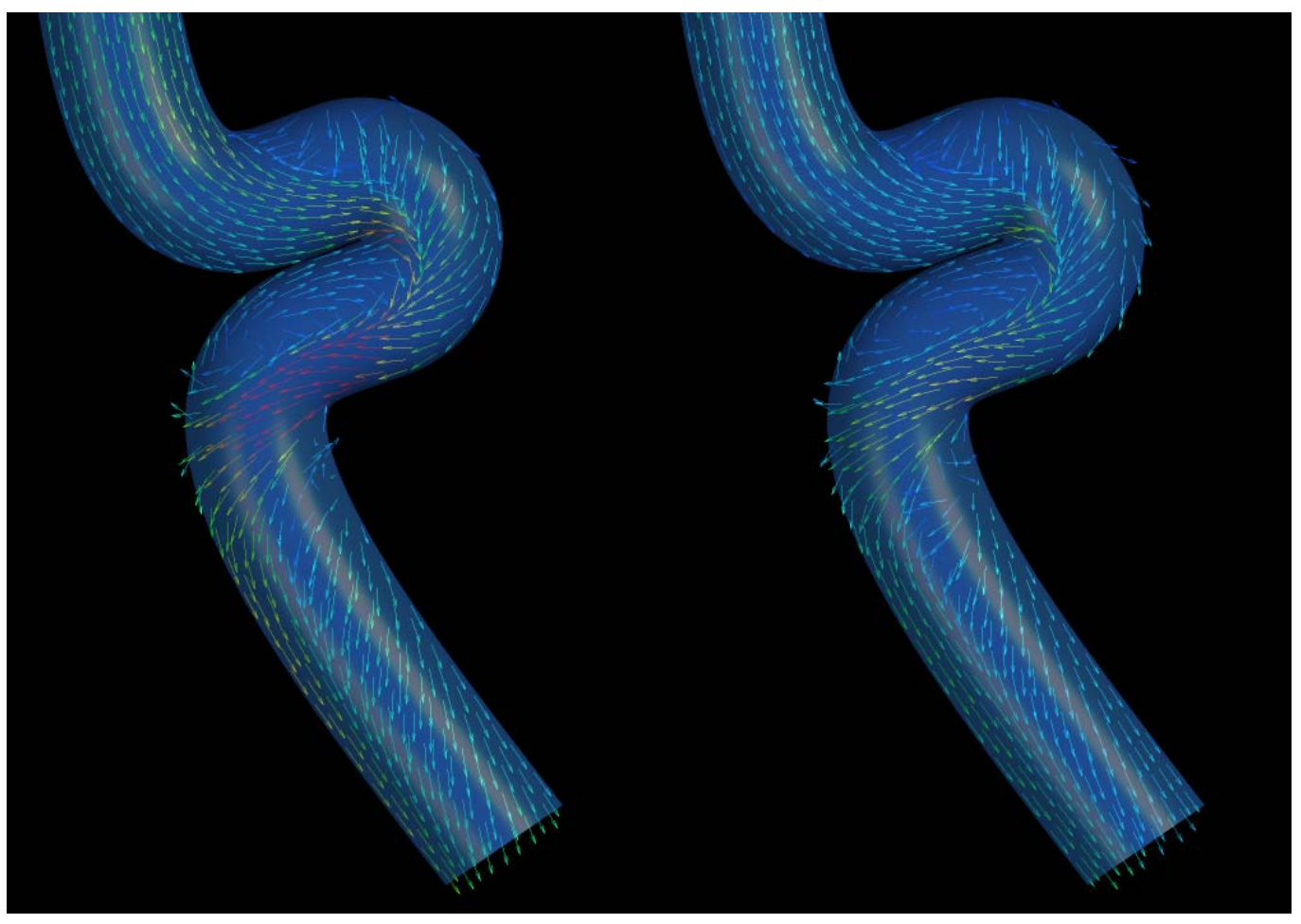

Figure 26: Velocity vectors on mid-coronal $(z=0)$ plane for severe-redundancy configuration under equal-mass flow (left) and equal-pressure (right) conditions. Note recirculation zone downstream of curvature; no recirculation zones near outlet boundary; red arrows seen on left indicate velocities are greater (see legend in Fig. 24 for values).

\subsection{Computational steady-state flow - Limb only}

The results for the limb component separation forces for two different boundary conditions of equal-mass flow or equal-pressure at the outlet of the limbs are summarized in Table 2.

Table 2: $\quad$ Limb Component Separation Force for Steady-State Simulations

Geometric Configuration

Limb component separation force (N)
Equal outlet mass flow

0.58

0.66

1.04
Severe-redundancy

Zero-redundancy

Moderate-redundancy
Equal outlet pressure 


\subsection{Computational pulsatile flow}

The predicted transient downward forces in three different geometries are presented in Figs. 27 and 28 for the equal outlet mass flow and equal outlet pressure conditions as a function of time, respectively. The peak downward displacement forces for the equal outlet mass flow studies occurs at peak systole, and are 7.35, 7.41 and $7.85 \mathrm{~N}$ for the zero-, moderate-, and severe-redundancy configurations, respectively. The peak downward displacement forces for the equal outlet pressure studies occurs at peak systole, and are 7.55, 7.70, and $8.31 \mathrm{~N}$ for the zero-, moderate-, and severe-redundancy configurations, respectively. The difference between the results due to differing outlet boundary conditions are especially accentuated in these plots of force over time.

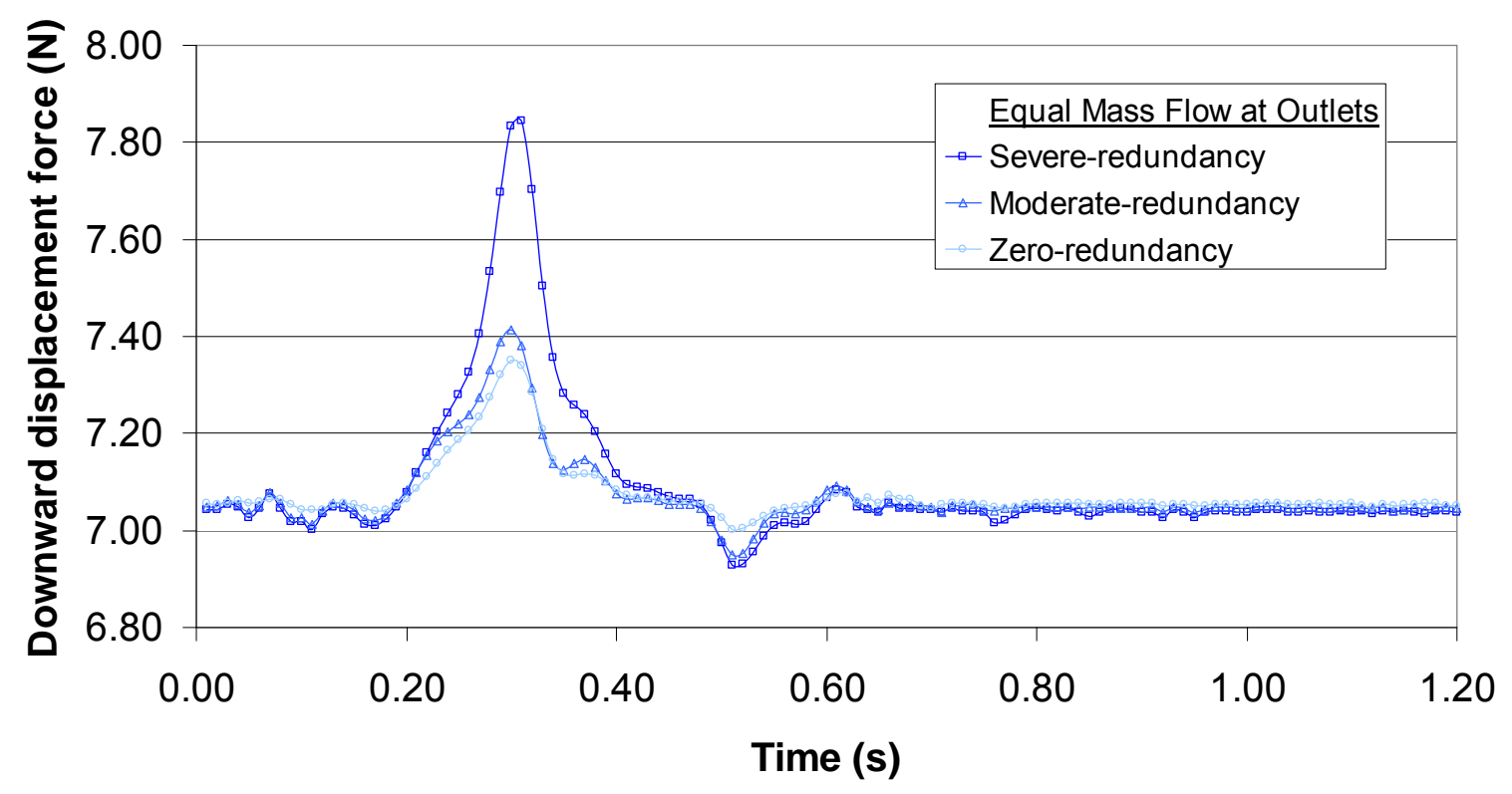

Figure 27: Plot of downward displacement force (in Newtons) over time (in seconds) for severe-redundancy (dark blue squares), moderate-redundancy (light blue triangles), and zeroredundancy (pale blue circles) configurations for equal-mass flow outlet condition. 


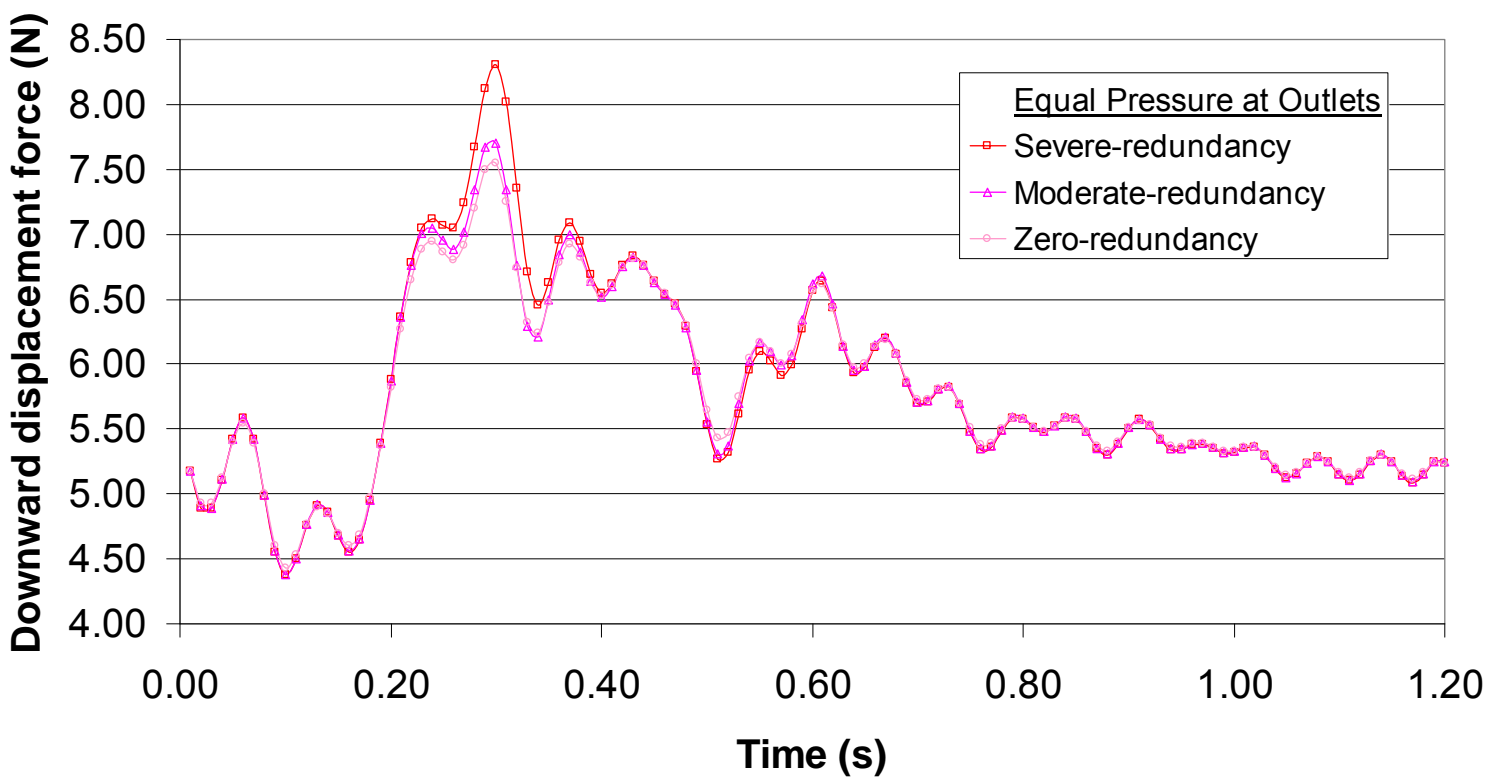

Figure 28: Plot of downward displacement force (in Newtons) over time (in seconds) for severe-redundancy (dark red squares), moderate-redundancy (light red triangles), and zeroredundancy (pale red circles) configurations for equal-pressure outlet condition.

The forces during peak systole are significantly higher than the rest of diastole, so that the relevant segment of the cardiac cycle is during forward, accelerating flow in systole and not during the reversed flow phases that occur during diastole (where the solution may be more prone to error). The greatest difference between the three geometries occurred during peak systole, while the forces were quite close during diastole.

Figure 29 illustrates the velocity vectors on the mid-coronal $(\mathrm{z}=0)$ plane during key time points of the cardiac cycle for the moderate-redundancy configuration with the equal outlet mass flow condition. Flow reversal is clearly visualized in Fig. 29(d), emphasizing the importance of allowing reversed flow across the outlet boundary. 


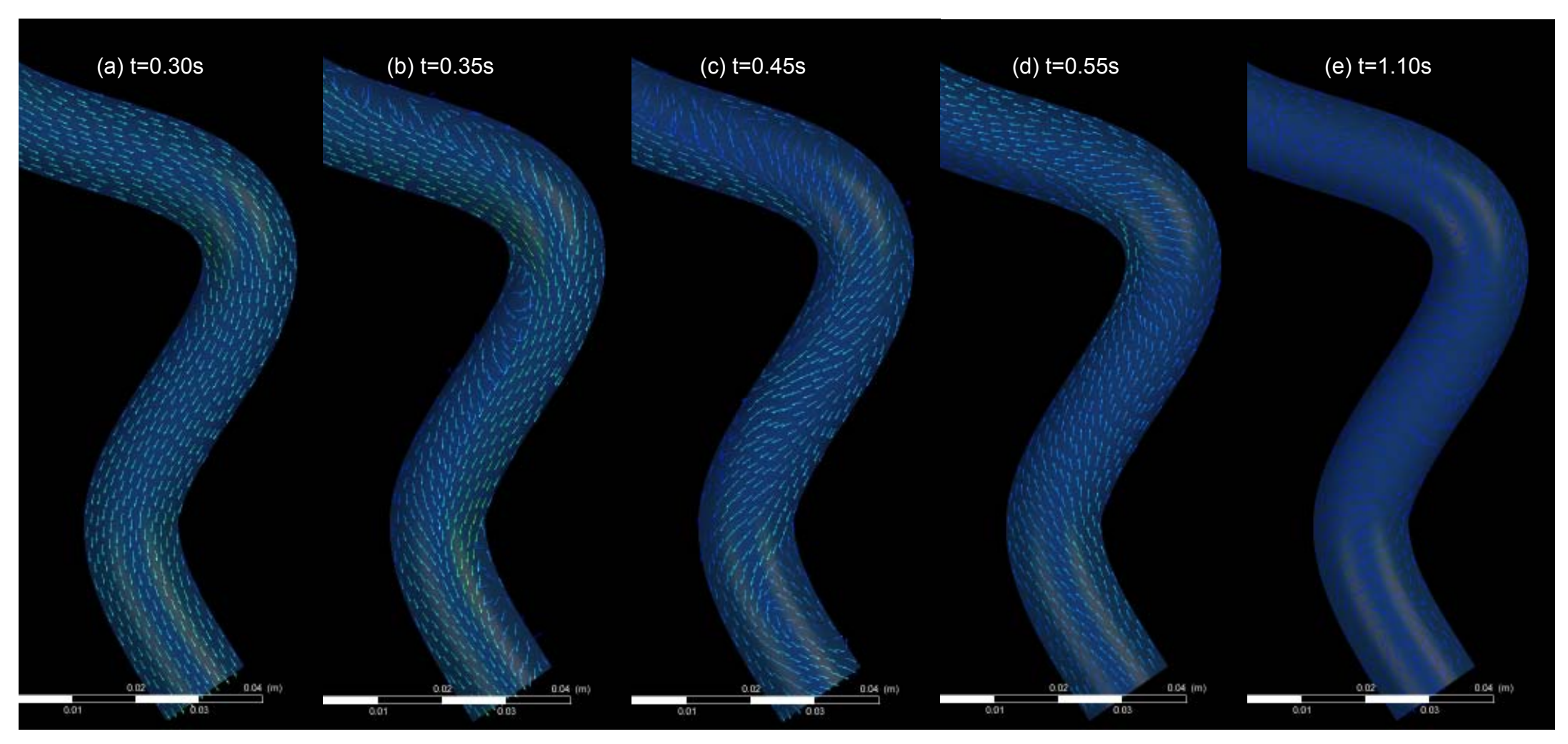

Figure 29: Velocity vectors on mid-coronal $(\mathrm{z}=0)$ plane for moderate-redundancy configuration with equal-mass flow outlet condition at a) $0.30 \mathrm{~s}$ (peak systole), b) $0.35 \mathrm{~s}$ (early deceleration), c) $0.45 \mathrm{~s}$ (late deceleration), d) $0.55 \mathrm{~s}$ (flow reversal), and e) $1.10 \mathrm{~s}$ (late diastole). 


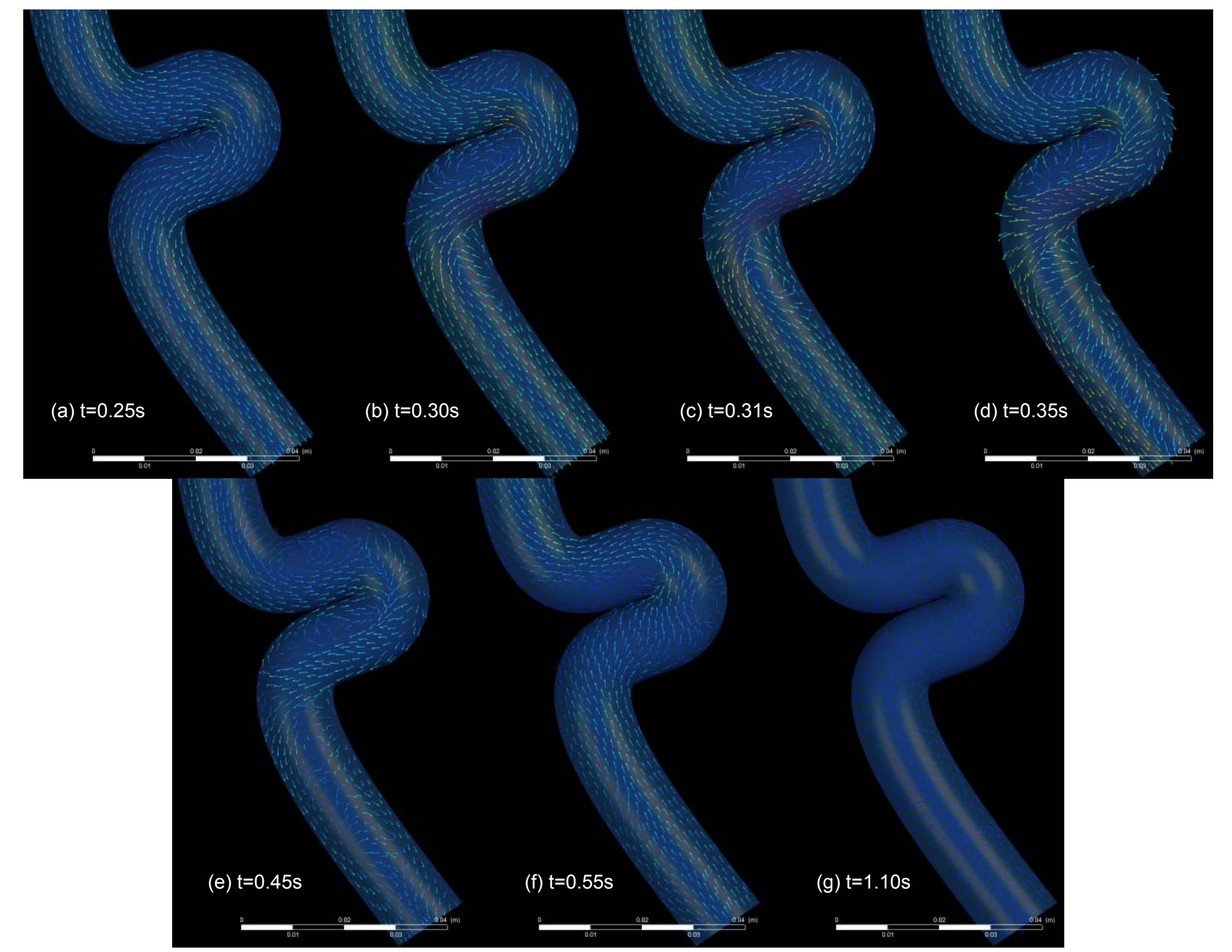

Figure 30: Velocity vectors on mid-coronal $(\mathrm{z}=0)$ plane for severe-redundancy configuration with equal-mass flow outlet condition at a) $0.25 \mathrm{~s}$ (acceleration phase), b) $0.30 \mathrm{~s}$ (peak systole), c) $0.31 \mathrm{~s}$ (just after peak systole), d) $0.35 \mathrm{~s}$ (early deceleration), e) $0.45 \mathrm{~s}$ (late deceleration), f) $0.55 \mathrm{~s}$ (reversal of flow), and g) $1.10 \mathrm{~s}$ (late diastole). 
Figure 30 illustrates the velocity vectors in the mid-coronal $(\mathrm{z}=0)$ plane during key time points for the severe-redundancy configuration with the equal outlet mass flow condition. The development and subsequent dissipation of different recirculation zones over time is visualized.

\subsection{Computational pulsatile flow - Limb only}

As illustrated in Fig. 2, the overall stent-graft device is composed of modular components that are held together by friction-fit. This is achieved by deploying the cylindrically-shaped stent-graft limb within the cylindrical component of the main-body component with sufficient "overlap" to prevent the components from separating. The force, due to blood flow, acting on the iliac limb to separate it from the main-body component is estimated from the pulsatile simulations.

The predicted transient limb component separation forces for the three different geometries are presented in Figs. 31 and 32 for the equal outlet mass flow and equal outlet pressure conditions, respectively. The peak limb component separation force for the equal outlet mass flow studies occurs at peak systole, and is $0.60,0.63$ and $1.09 \mathrm{~N}$ for the zero-, moderate-, and severe-redundancy configurations, respectively. The peak limb component separation force for the equal outlet pressure studies occurs at peak systole, and is $0.58,0.64$, and $0.92 \mathrm{~N}$ for the zero-, moderate-, and severe-redundancy configurations, respectively. 


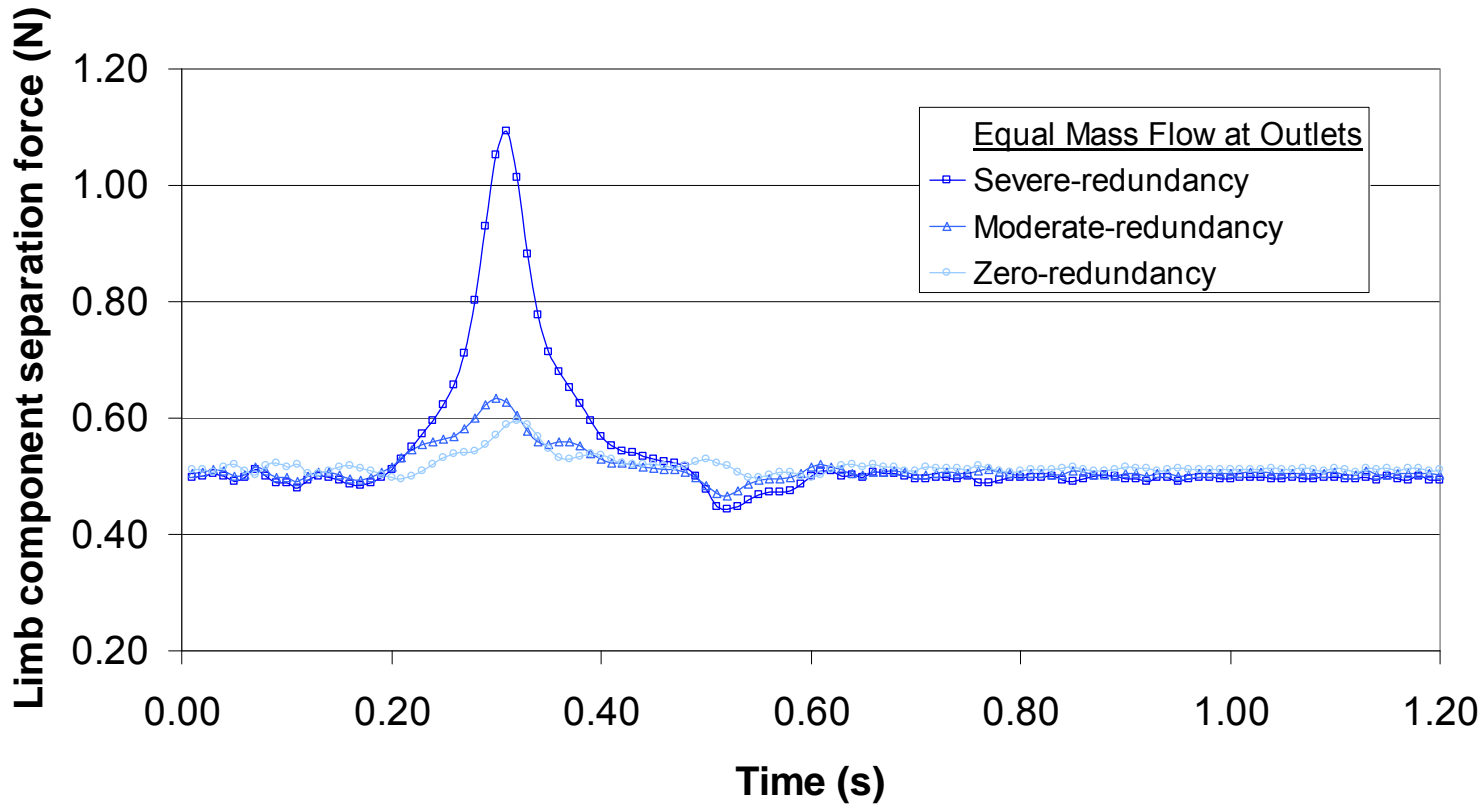

Figure 31: Plot of limb component separation force (in Newtons) over time (in seconds) for severe-redundancy (dark blue squares), moderate-redundancy (light blue triangles), and zero-redundancy (pale blue circles) configurations for equal-mass flow outlet condition.

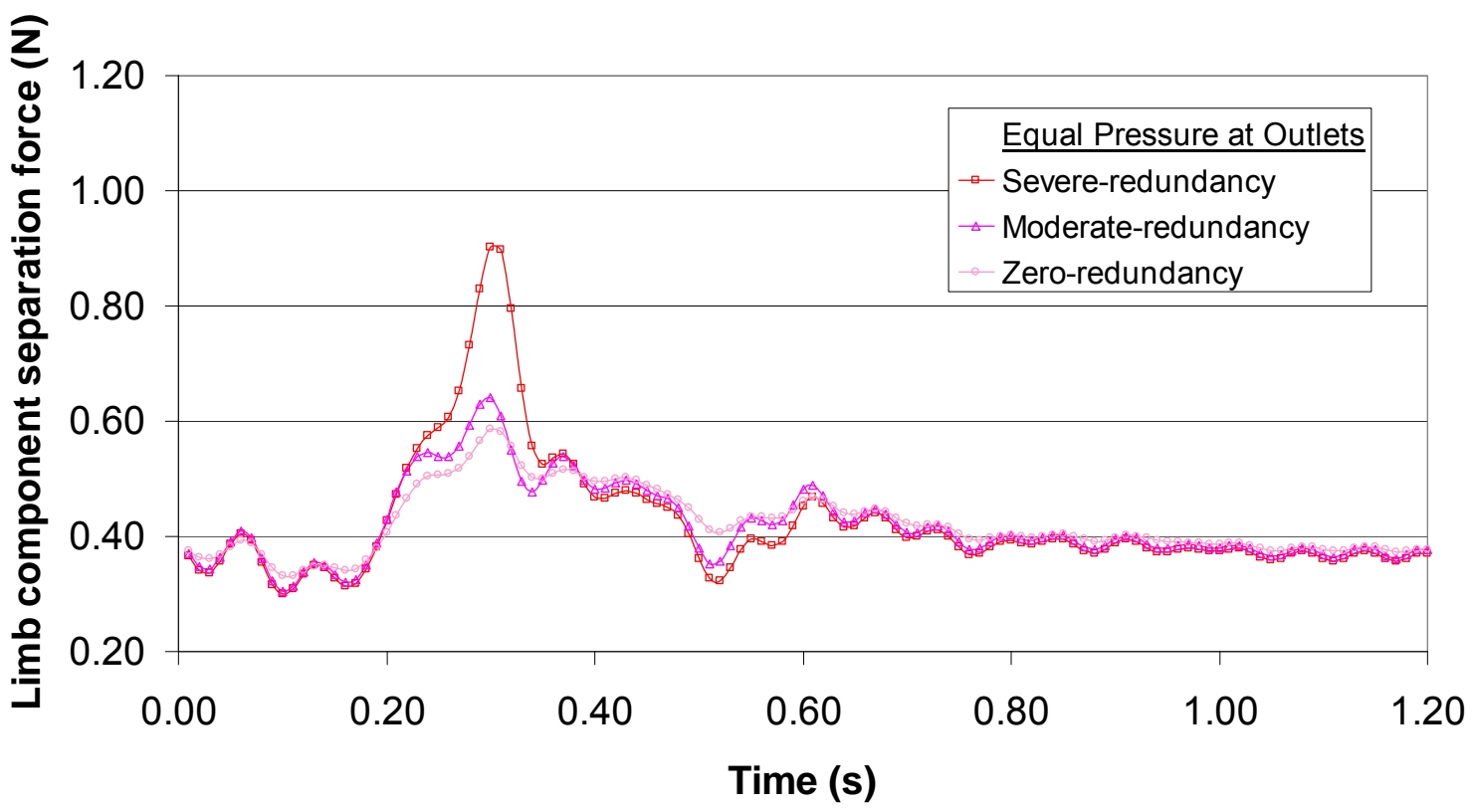

Figure 32 Plot of limb component separation force (in Newtons) over time (in seconds) for severe-redundancy (dark red squares), moderate-redundancy (light red triangles), and zero-redundancy (pale red circles) configurations for equal-pressure outlet condition. 
These values are much smaller than the forces acting on the entire device, indicating that the main-body portion of the device (rather than the limb) is subjected to the majority of the overall displacement force. The main-body of the device is anchored to the aorta through a stronger graft-to-aorta interaction, whereas the component-to-component limb attachment is only through weaker graft-to-graft friction-fit interaction. Therefore the resistance is much less than for the main-body (discussed in section 4.2).

\subsection{Multiple cycles and artificial walls}

As mentioned in section 2.8, the entire solution domain was initialized to zero velocity. This initialization would only concern the first cycle of the transient simulation, while the subsequent cycles would use the previous cycle solution as the initial condition. To study the effect of the initialization on the predicted results, we simulated transient flow in the moderate-redundancy configuration for two cycles. Figure 33 shows the moderateredundancy configuration (equal outlet pressures) with the first and second cycles overlapped. Although there is slight difference at the beginning of the cycle, the simulated forces quickly become near-identical. Given these results, subsequent simulations were carried out over a single cardiac cycle. 


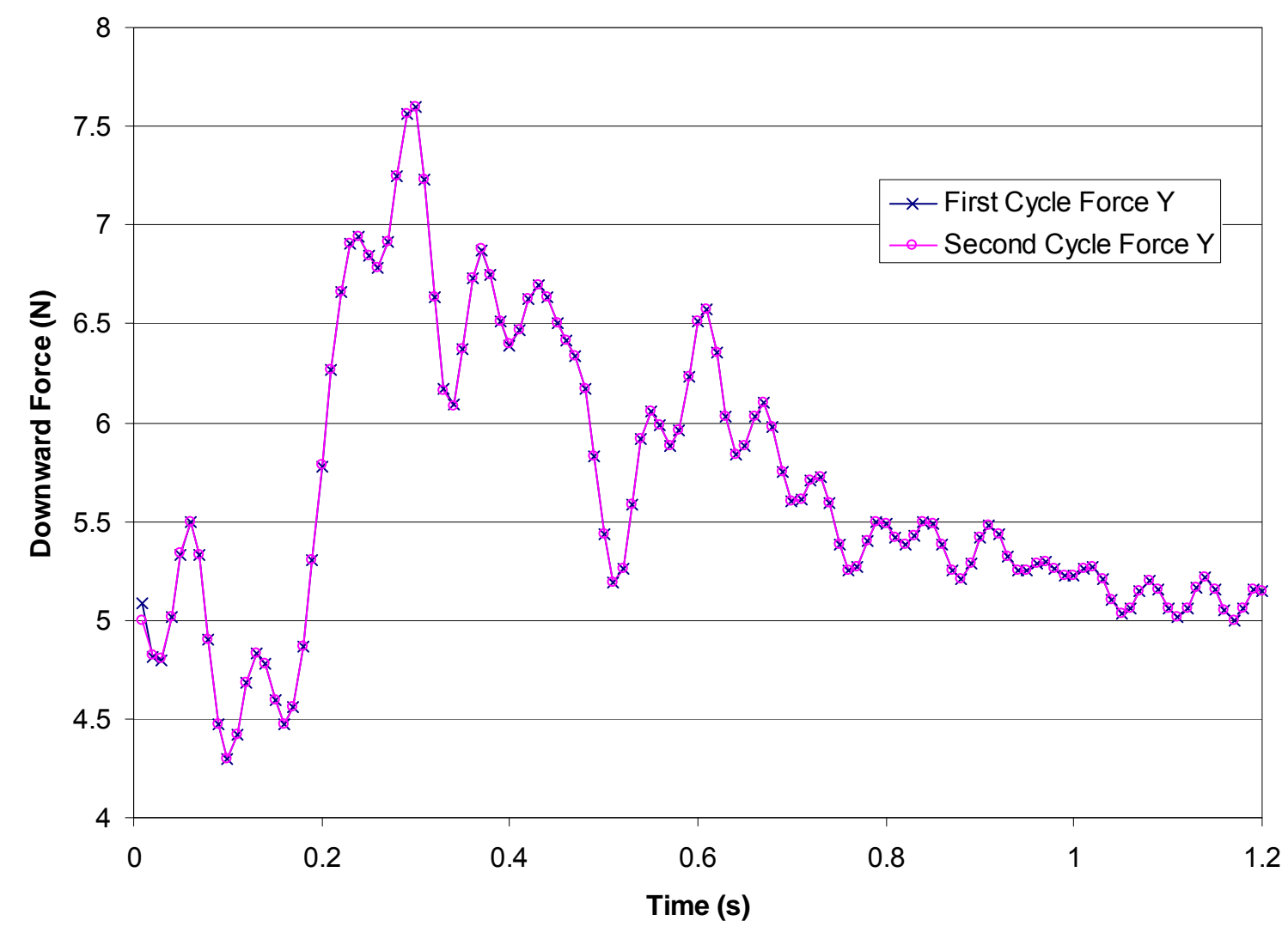

Figure 33: Plot of downward force for two cardiac cycles simulated for the moderateredundancy configuration that are shown overlapped. Note the small difference at the beginning of the cycle that quickly disappears.

When using mass flow as an outlet boundary condition, the software Ansys CFX will normally erect "artificial walls" when it detects "negative" velocity vectors on the outlet boundary (i.e., flow directed inwards). The artificial walls are erected by changing these 'negative' velocities on the outlet boundary to a value of zero [31]. However, our pulsatile scenario calls for flow reversal during the cardiac cycle. Therefore, we turned off the artificial walls for our simulation. Figure 34 is a plot of the downward force for equal outlet mass flow with artificial walls (red squares) and without artificial walls (green circles). This demonstrates that during the relevant phase of the cardiac cycle (peak systole), there is little difference whether the "artificial wall"-function of the commercial solver is applied or not. 
This is because the artificial walls are only erected during diastole when there is a reversal of flow. It is clear that the artificial walls do not handle the reversal of flow appropriately.

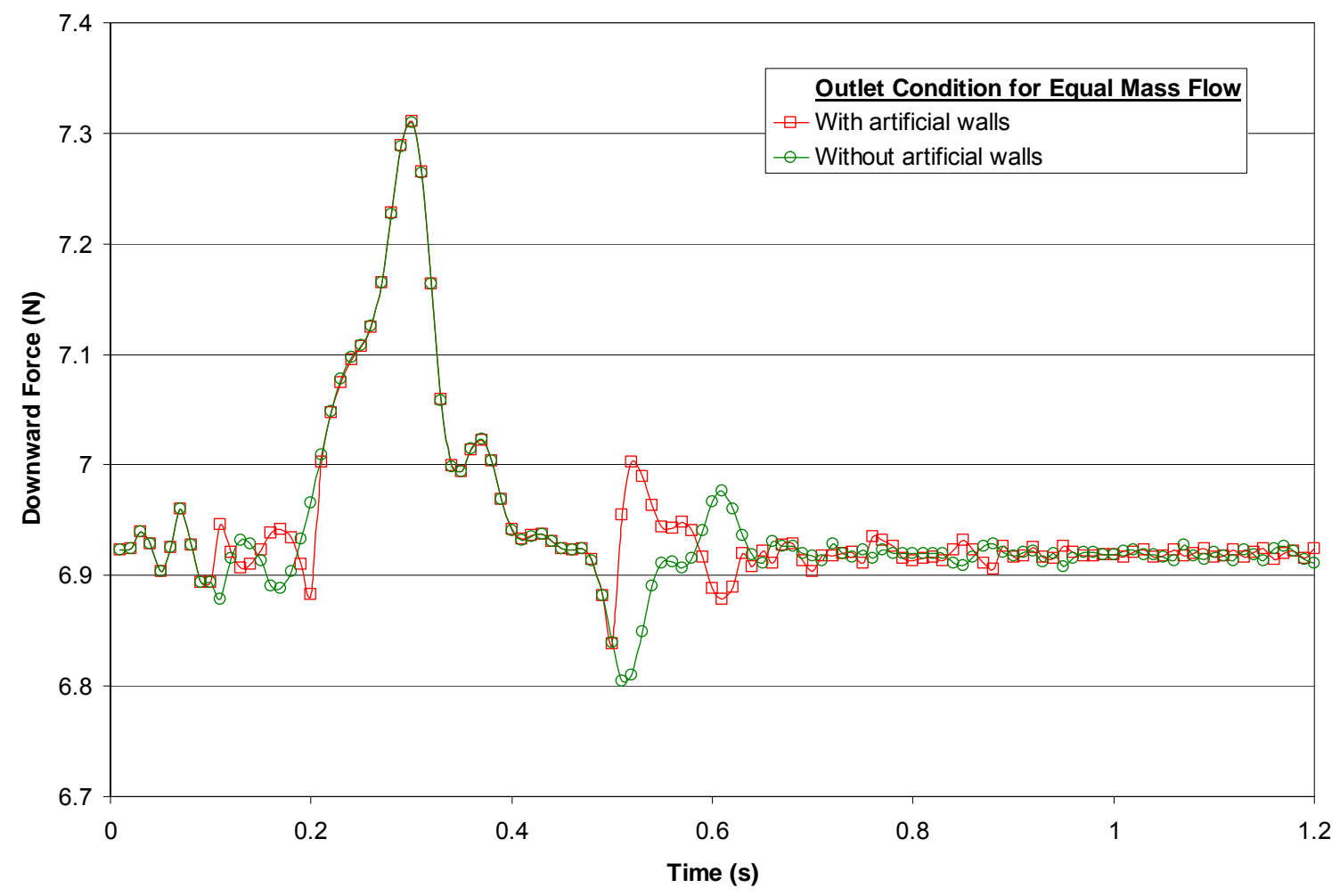

Figure 34: Plot of downward force for equal outlet mass flow with artificial walls (red squares) and without artificial walls (green circles) when there is flow reversal at the outlets.

Figure 35 illustrates the velocity vectors with and without artificial walls during initiation of the flow reversal phase $(\mathrm{t}=0.52 \mathrm{~s})$ and at full flow reversal $(\mathrm{t}=0.55 \mathrm{~s})$. The solver had erected artificial walls over $56 \%$ of the outlet boundary during its first iteration of $t=0.52 \mathrm{~s}$. One can clearly see that during full flow reversal at $\mathrm{t}=0.55 \mathrm{~s}$, there is inappropriate forward-directed flow in the model that used artificial walls. 


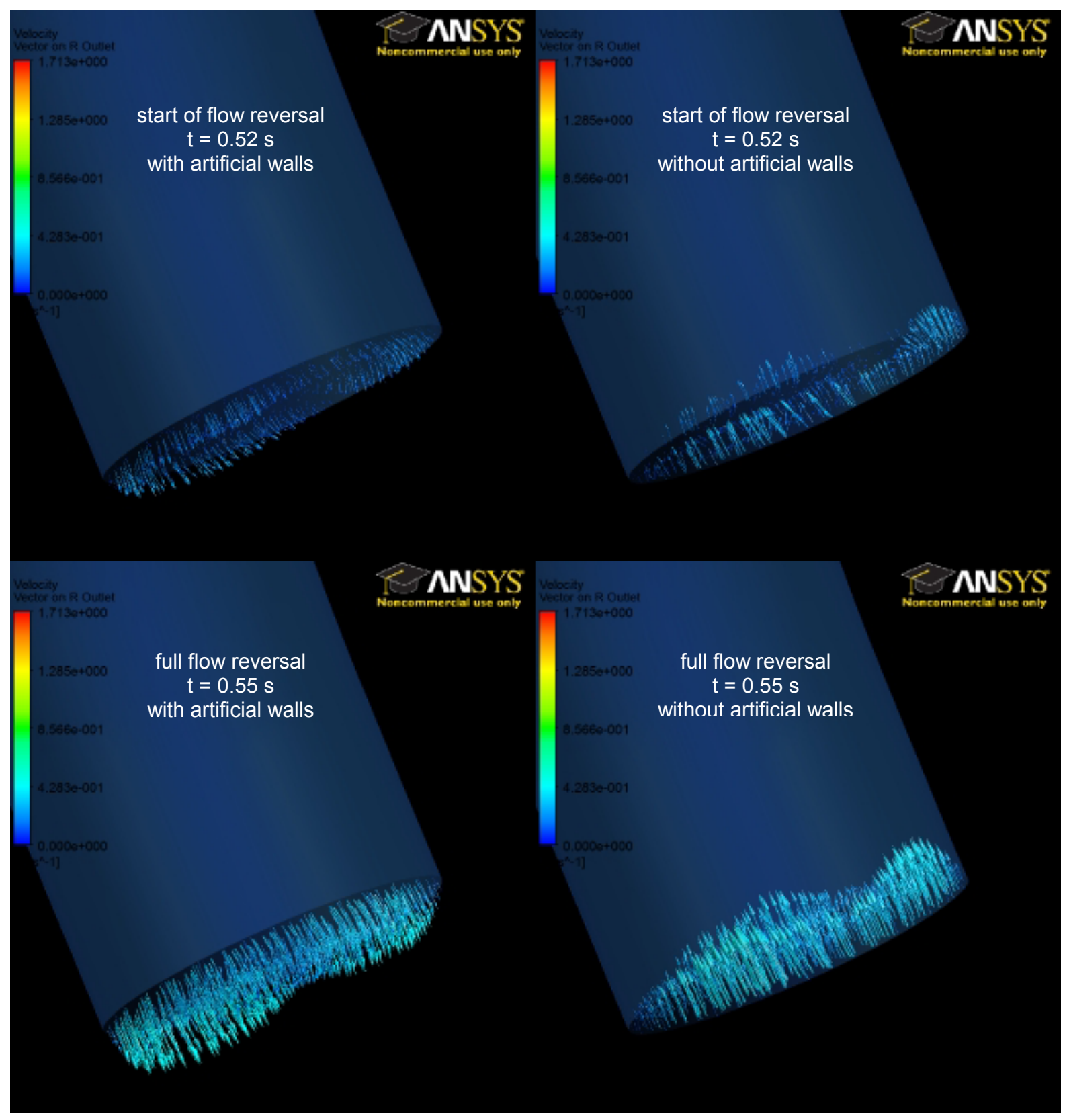

Figure $35 \quad$ Velocity vectors on the outlet boundary, with artificial walls (left panels) and without artificial walls (right panels) during initiation of the flow reversal phase $(\mathrm{t}=0.52 \mathrm{~s}$, upper panels) and during full flow reversal $(t=0.55 \mathrm{~s}$, lower panels). The solver had erected artificial walls over $56 \%$ of the outlet boundary during its first iteration of $t=0.52 \mathrm{~s}$. One can clearly see that during full flow reversal at $\mathrm{t}=0.55 \mathrm{~s}$, there is inappropriate forward-directed flow in the model that used artificial walls. 
On the other hand, Fig. 36 is a plot of the RMS of residuals, which indicates that without the artificial walls, the solution is not as stable/robust in the late diastolic phase. The implications are reviewed in the discussion (Section 4.5).

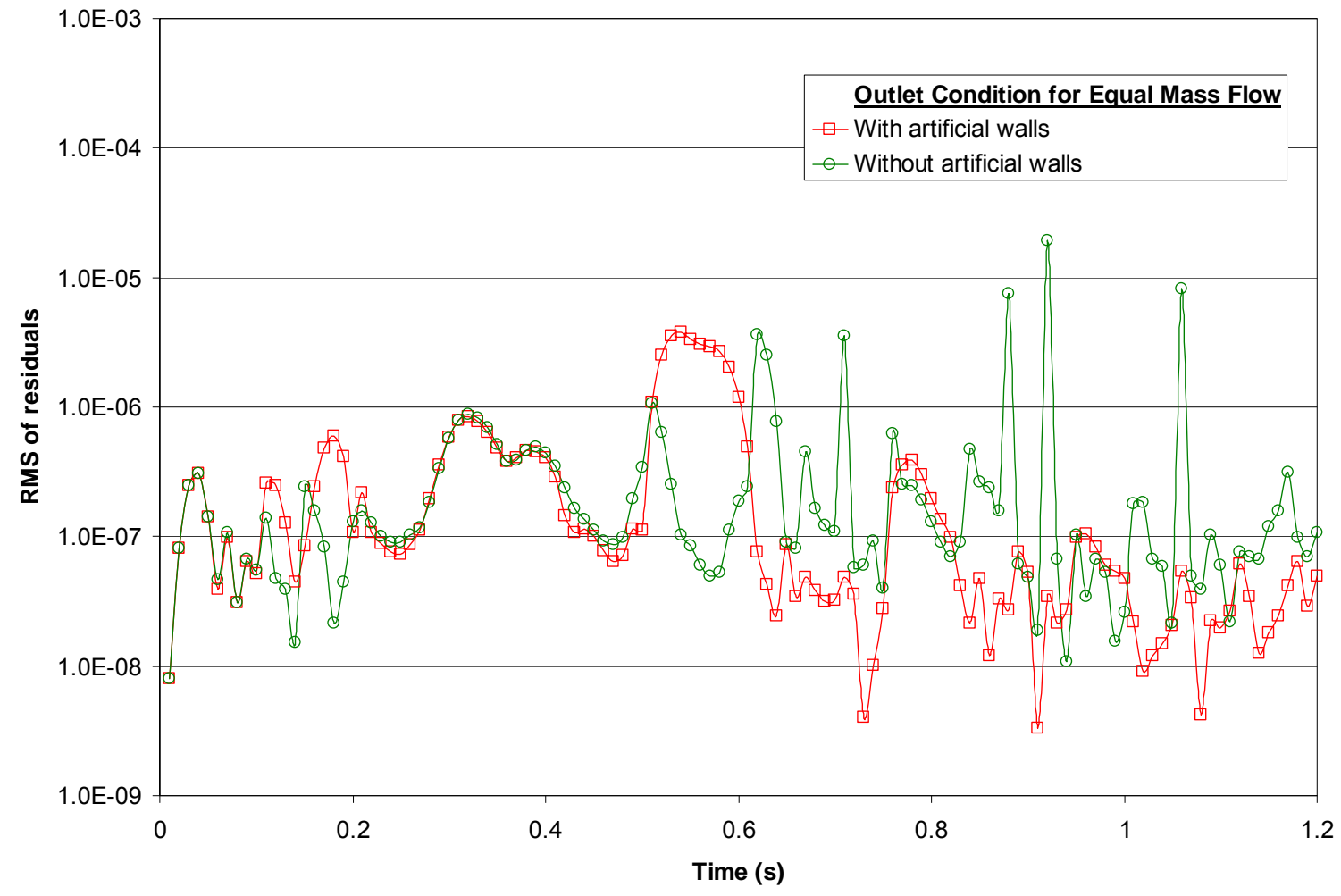

Figure 36: Plot of values for root mean square (RMS) of residuals for equal outlet mass flow with artificial walls (red squares) and without artificial walls (green circles) when there is flow reversal at the outlet. 


\section{Discussion}

To our knowledge there is no published study that evaluates redundant endovascular stentgraft configurations. This research employed analytical and CFD methods to study the blood flow in redundant configurations and investigate the effect of redundancy on the predicted forces.

\subsection{Clinical relevance}

The results of this work confirm that increasing limb redundancy increases the displacement force acting on endovascular stent-grafts. However, the order of magnitude of the increase is not clinically significant. Specifically, the magnitude of the change is almost one order of magnitude smaller than the overall force acting on the device. The results suggest that the practice of forming a redundant segment of stent-graft limb in the aneurysm sac to accommodate extra length does not appear to significantly affect the dislodgement force.

However, our results raise other clinical concerns. Our predicted displacement forces echo those of other CFD studies which report values in the range of 4 to $10 \mathrm{~N}$, as shown in Table 3. Experimental studies have indicated that older generations of stent-grafts (prior to the use of large barbed suprarenal stents) can only resist displacement forces of 2.5 to $8.0 \mathrm{~N}$ [32-34]. The results of these studies are summarized in Fig. 37. 


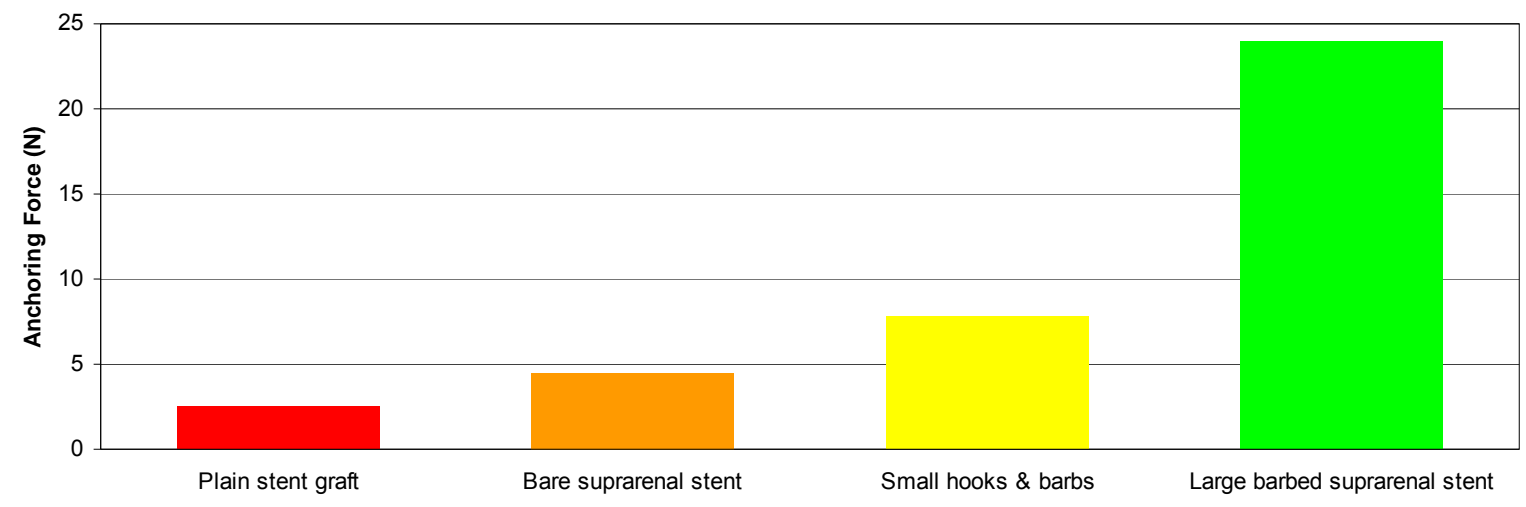

Figure 37: Graph comparing anchoring force (in Newtons) for various proximal fixation strategies for stent grafts, from references [32-34].

This would suggest that older generations of stent-grafts are at significant risk of dislodgement. Dislodgement of the device could subsequently result in pressurized blood flowing into the aneurysm sac again. Newer generations of stent-grafts were reported to be able to resist displacement forces of up to $24 \mathrm{~N}[33,34]$, which offered a significant safety margin against device migration and displacement. However, a very recent paper has suggested that even some of the newer-generation stent-grafts can only resist forces of $6 \mathrm{~N}$ [35]. Further experimental studies of the resistance of stent-grafts to displacement will be helpful in better delineating the risks of device-specific displacement.

\subsection{Limb component separation}

The results from evaluation of the effects of redundancy on limb component separation force were similar to those of the entire device. While increasing redundancy resulted in increasing force, the magnitude of the increase was small. The absolute values for the estimated limb component separation force for this work are within the limits for experimentally-derived published values for device-specific resistance to limb component separation $[34,36]$. The experimental values from Cina et al. and Hinchliffe et al. for the force that is required to separate the limbs for different commercial devices are summarized in Fig. 38. 


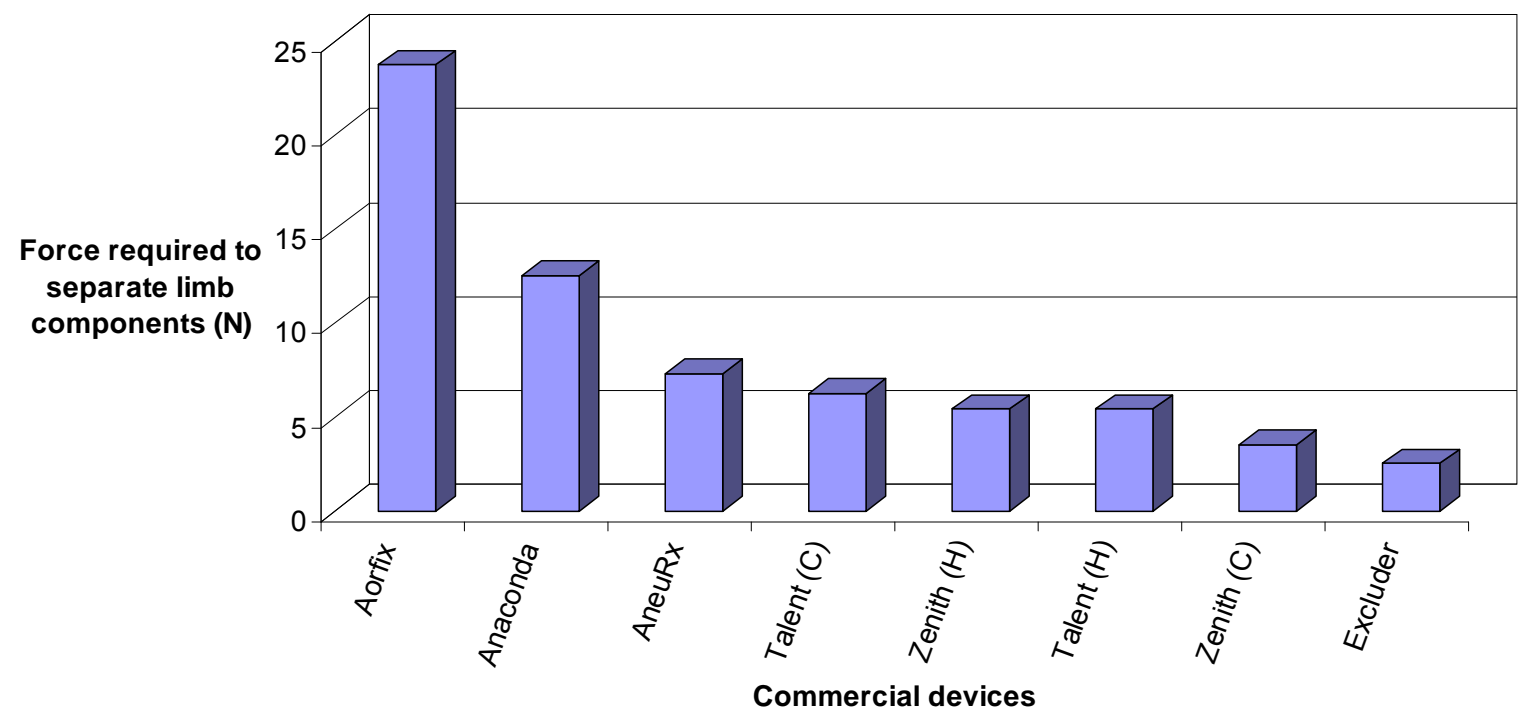

Figure 38: Graph of experimentally-derived forces required to separate limb components for various commercial devices. $(\mathrm{C})=$ from Cina et al. [36] $(\mathrm{H})=$ Hinchliffe et al. [34].

The lowest value for the experimental forces was $2.5 \mathrm{~N}$, which is well above the maximum predicted value of only $1.09 \mathrm{~N}$ for the most extreme case in our study.

\subsection{Validity}

While predictions of clinical results would be an ideal goal of CFD studies for stent-grafts, there is no reasonable technique available for measurement of displacement forces in clinical models to validate these CFD studies. The trends identified by CFD studies as factors contributing to increased displacement force (e.g., increased proximal diameter, high blood pressure, increased bifurcation angle, etc.) have also been noticed in studies investigating factors contributing to clinical cases of device migration [9]. However, one cannot apply causation based on the similarity of observed trends. There are very few experimental in vitro studies available to which we can compare CFD results, and the studies that are available do not use parameters and conditions comparable to those that CFD studies attempt to simulate 
$[23,37]$. Even within the literature on CFD of stent-graft blood flow, the geometries (dimensions) as well as the boundary conditions and blood physical properties vary widely, as presented in Tables 1 and 3 .

Table 3: $\quad$ Parameters and results of other CFD studies of stent-grafts.

\begin{tabular}{|c|c|c|c|c|c|c|c|}
\hline Author & $\begin{array}{l}\text { Density } \\
\left(\mathrm{kg} / \mathrm{m}^{3}\right)\end{array}$ & $\begin{array}{c}\text { Dynamic } \\
\text { Viscosity } \\
(\mathrm{Pa} \cdot \mathrm{s}) \\
\end{array}$ & $\begin{array}{c}\text { Inlet } \\
\text { (Peak Vel./ } \\
\text { Vol. Flow) }\end{array}$ & $\begin{array}{c}\text { Outlet } \\
\text { Pressure } \\
(\mathrm{mmHg})\end{array}$ & $\begin{array}{c}\text { Steady } \\
\text { vs. } \\
\text { Transient }\end{array}$ & $\begin{array}{l}\text { Laminar } \\
\text { vs. } \\
\text { Turbulent }\end{array}$ & $\begin{array}{c}\text { Downward } \\
\text { Force } \\
\text { (N) }\end{array}$ \\
\hline Present work & 1050 & 0.0035 & $0.53 \mathrm{~m} / \mathrm{s}$ & $80-120$ & Both & Both & $7.36-8.31$ \\
\hline \multicolumn{8}{|l|}{ Closest Models } \\
\hline$\overline{\text { Morris et al. [7] }}$ & 1050 & 0.0035 & $0.53 \mathrm{~m} / \mathrm{s}$ & $80-120$ & Pulsatile & Laminar & $\sim 8.0$ \\
\hline Li et al. $[20,21]$ & 1050 & Non-N & $0.22 \mathrm{~m} / \mathrm{s}$ & $80-120$ & Pulsatile & Laminar & $\sim 6.2$ \\
\hline \multicolumn{8}{|l|}{ Other $30 \mathrm{~mm}$} \\
\hline \multicolumn{8}{|l|}{ Diameter Models } \\
\hline$\overline{\text { Liffman et al. [5] }}$ & 1060 & 0.0036 & $0.6 \mathrm{~m} / \mathrm{s}$ & 100 & Steady & Turbulent & 7.12 \\
\hline Liffman et al. [22] & 1060 & ns & $10.4 \mathrm{~L} / \mathrm{min}$ & $80-130$ & Pulsatile & Turbulent & 10 \\
\hline Finol et al. [6] & 1050 & 0.00319 & $0.6 \mathrm{~m} / \mathrm{s}$ & 120 & Steady & Turbulent & 9 \\
\hline Zhou et al. [23] & 1098 & 0.0033 & $1.5 \mathrm{~L} / \mathrm{min}$ & $60-160$ & Pulsatile & Exprmntl & 5.5 \\
\hline \multicolumn{8}{|l|}{ Other Studies } \\
\hline Frauenfelder et al. [16] & 1060 & 0.0035 & $90 \mathrm{~m} / \mathrm{s}$ & ZTF & Pulsatile & Laminar & ns \\
\hline Howell et al. [14] & ns & $\mathrm{ns}$ & $0.77 \mathrm{~m} / \mathrm{s}$ & Mss Flw & Pulsatile & ns & $2-6$ \\
\hline Molony et al. [15] & 1050 & 0.0035 & $0.53 \mathrm{~m} / \mathrm{s}$ & $80-120$ & Pulsatile & Laminar & 5 \\
\hline Morris et al. [24] & 1050 & 0.0035 & 3.7 L/min & 120 & Steady & ns & 4.2 \\
\hline
\end{tabular}

* Non-N = non-Newtonian; $\mathrm{ns}$ = not specified; Mss Flw = mass flow was specified as outlet boundary condition; $\mathrm{ZTF}=$ zero-traction force

However, our results are consistent with those of other published authors. The study with the closest dimensions is that from Morris et al., where the predicted downward displacement force was $8.0 \mathrm{~N}$ [7]. Li et al. also had a proximal diameter of $30 \mathrm{~mm}$ that predicted a force of $6.2 \mathrm{~N}$ [21]. The work of Liffman et al. indicated that a proximal diameter of $30 \mathrm{~mm}$ would give a force of $7.12 \mathrm{~N}$ [5]. Zhou et al. conducted experimental work with a $30 \mathrm{~mm}$ proximal diameter and determined a displacement force of $5.5 \mathrm{~N}$ [23], but comparison to this case is difficult because the remaining geometric dimensions were not specified and the volumetric flow, pressure, and fluid properties were different. All of the previous published results using 
CFD with stent-graft dimensions similar to our study are within an appropriate range of our results that falls between $7.36 \mathrm{~N}$ and $8.31 \mathrm{~N}$.

More importantly, the goal of this study was to determine the effect of redundancy, rather than predicting the absolute values of displacement forces. The focus of this work was determining the magnitude of the change in force due to change in redundancy. The order of magnitude of change is significantly smaller than that of the overall force acting on the device. Therefore, we are comfortable in interpreting the results as suggesting that while redundancy does increase the displacement force, it is not of sufficient magnitude to be clinically relevant.

\subsection{Limitations}

Significant simplifications were applied to arrive at our solutions. These simplifications included the geometries constructed as well as the graft properties and boundary conditions.

The geometries used were very idealized with respect to shape and symmetry. While our limb cross-sections were perfect circles, in clinical reality, the shapes are much more eccentric and irregular. Even within the flow-channel, we have not included the effects of the stents, or how the fabric may be crimped or folded. In reality, there may also be a variable layer of thrombus (clotted blood) that covers the inside of the graft. The geometry that we chose to represent redundancy is also idealized, as patient-specific anatomy is unlikely to exactly follow a $45^{\circ}$-bend, followed by a $90^{\circ}$-bend, and then another $45^{\circ}$-bend, as we had created for our moderate-redundancy configuration. However, the goal was to evaluate the effects of increasing redundancy from a general point of view, rather than to obtain exact absolute values for patient-specific models. For this reason, idealized geometries were used to allow better control of the variable in question, redundancy.

The properties of the graft were also simplified. The graft was treated as a rigid pipe, rather than a compliant, deformable tube. The addition of graft compliance to our simulation would require coupling the behaviour and properties of the graft with the behaviour and properties 
of the fluid, as in fluid-structure interaction (FSI) analysis. Given that the graft material is relatively non-compliant [38], so that it does not deform significantly through the cardiac cycle, it is suspected that the increased computational requirements of FSI techniques would add significantly to the accuracy of the results.

Finally, the boundary conditions were also simplified. We used a simple paraboloid velocity profile at the inlet. While Morris et al. have reported that the inlet velocity profile does not significantly affect the flow in stent-grafts [24], a less-developed (i.e., blunter) profile may be closer to clinical reality. The two different outlet boundary conditions used in this study were also simplified. While we used either specified pressure or specified mass flow, other authors are aggressively pursuing very sophisticated methods to simulate outlet conditions that are meant to account for the large effects of the downstream peripheral vascular bed [39].

\subsection{Outlet boundary conditions - equal pressure vs. equal mass flow}

For published CFD studies of endovascular stent-grafts, equal pressure at the outlet of the two limbs is commonly used as the outlet boundary [5, 7, 15, 20-22, 24, 39]. For the commercial software Ansys CFX, it is recommended that the most robust conditions are velocity-specified inlet and pressure-specified outlets [26]. This equal outlet pressure boundary condition was applied to the aforementioned studies, even when the outlet limbs were asymmetric $[5,15,24]$. However, in our scenario of an asymmetric bifurcated graft, the use of equal outlet pressures seemed to exaggerate the flow distribution. This is because the viscous effects over the length of the graft walls are the primary contribution to the resistance to flow, whereas in reality, these effects are significantly less than those of the downstream vasculature of the leg. We therefore also carried out simulations that imposed velocityspecified inlet, and equal mass flow specified at the two limb outlets. For the commercial software Ansys CFX, this is considered to be the next-most-robust set of boundary conditions [26]. While the equal outlet mass flow condition addressed the exaggerated flow distribution, this 50:50 division is also a non-physical assumption; and the appropriate solution likely lies somewhere between equal-pressure outlets and equal-mass flow outlets (but probably closer to the side of equal-mass flow). 
Despite the different boundary conditions that were applied, the peak forces for equalpressure and equal-mass flow were quite close. However, the amplitude of variation in the cardiac cycle was significantly larger for the equal pressure condition. This amplitude, rather than peak force, may be of significance in the evaluation of fatigue, since fatigue failure is heavily influenced by the amplitude of the load cycle. Fortunately, the true-life scenario is closer to the equal mass flow condition, which experiences a smaller change in the force amplitude, and therefore more likely to fare well with cyclic fatigue.

One significant problem with setting equal mass flow as the outlet boundary condition is that the commercial software is not clear on how to deal with reversal of flow. The default setting is to setup artificial walls at the outlet boundary to prevent flow from entering the graft through the outlet (only when there is flow reversal). It does this to maintain as robust a solution as possible. However, it is non-physical and yields inappropriate results when the velocity profile clearly calls for flow reversal. Therefore, we used the advanced settings to turn off the artificial walls. As shown in Fig. 34, while there were clearly differences in the solutions for the diastolic phase, there was no difference for the systolic phase (since there was no flow reversal, and no recirculation zones near the boundary during the acceleration in systole). For our particular study, the most relevant phase was the systolic phase and therefore, this was not a significant issue for this work.

However, if the diastolic phase was important, then this method would not be sufficient. While extending the limbs would be helpful in dealing with recirculation at the outlet boundary, more sophisticated methods may be necessary to fully address flow reversal. Vignon-Clementel et al. have published work that attempts to more accurately define the outlet boundary condition using impedance, rather than pressure [39]. In their study, application of the equal outlet pressure condition to a bifurcated aortic model with unilateral iliac stenosis resulted in an exaggerated asymmetric flow distribution, similar to our present work. However, application of their impedance model (that involves the use of wave theory and fractal mathematics) resulted in almost equal mass flow at the outlets. Appropriate modeling of the boundary conditions remains an important area of study and development in bioengineering. 


\subsection{Lateral forces}

A recent study by Figueroa et al. has highlighted the fact that the important displacement forces are not entirely in the downward direction, but that a significant component is in the 'lateral' direction [18]. Since the geometry created in our study lay entirely in a horizontal plane, there was no lateral force in the z-direction (which was the direction of significant proximal anterior angulation in their study). However, the focus of our work was on the effect of redundancy and therefore the geometric configurations were meant to isolate the differences in results to the differences in redundancy. The significance of lateral, or out-ofplane, forces will likely be very important clinically and will be included in our group's next computational work on out-of-plane geometric configurations. We also hope to pursue experimental work to investigate device-specific resistance to out-of-plane displacement force. This will add important information to the limited number of experimental studies that CFD studies can be compared against.

\subsection{Single load versus fatigue}

Finally, it is important to keep in mind that the results presented here are for single load applications. It is well-known that cyclic, repetitive fatigue requires significantly smaller loads to result in failure. Given that physiologic flow and pressure usually cycle at greater than $1 \mathrm{~Hz}$, the stent-graft would be subjected to approximately 400 million cycles over 10 years of clinical service life. While it would be desirable to simulate this fatigue "in silico" or with bench-top experiments, this type of fatigue would be extremely difficult to model, given that the aorta is a "living pipe" that has the capacity to re-model and accommodate stresses and damage. 


\section{Conclusion}

This work demonstrates that redundant stent-graft configurations in EVAR do increase the downward force acting on the device and its modular components. However, the magnitude of increase in dislodgement force and limb component separation force is significantly smaller than the overall forces that the devices are subjected to. Given the wide variability in device-specific resistance to migration and separation, the clinical consequences would depend on the particular device used. Considering the high mortality rate of ruptured AAAs and the fact that device dislodgement can lead to device failure, stent-grafts with low resistance to displacement forces need to be monitored closely. 


\section{Future Work}

Experimental bench-top validation of these computational simulations would be helpful in supporting the conclusion of this study. With respect to clinical studies, while there is presently no method to measure the in vivo displacement force of a device that is already implanted in a patient, it may be beneficial to screen computed tomography images to determine the prevalence of limb redundancy in patients who have undergone EVAR. The prevalence would be expected to increase with newer generations of flexible stent-grafts, and as the practice of using redundancy to accommodated extra graft length is encouraged. Patients with redundancy could be followed and assessed for graft migration.

It is becoming increasingly clear that evaluating the simple downward (y-direction) force acting on the device is not sufficient, and that the out-of-plane forces play an important role $[18,40]$. Our group is presently evaluating the out-of-plane configuration of crossed-limb stent-grafts. Given that the recent studies indicate that forces in the anterior direction are significant $[18,40]$, our group plans to carry out experimental studies to determine pullout forces at various anterior and lateral angulations. These would be important contributions to the understanding of stent-grafts, and the experimental studies could serve as references against which predicted forces from simulations could be compared. 


\section{References}

[1] T. M. Mastracci and C. S. Cina, "Screening for abdominal aortic aneurysm in Canada: Review and position statement of the Canadian Society for Vascular Surgery," Journal of Vascular Surgery, vol. 45, pp. 1268-1276, Jun 2007.

[2] N. L. Volodos, V. E. Shekhanin, I. P. Karpovich, V. I. Troian, A. Gur'ev Iu, and I. A. Gur'ev, "[A selffixing synthetic blood vessel endoprosthesis]," Vestnik Khirurgii Imeni i - i - Grekova, vol. 137, pp. 123-5, Nov 1986.

[3] J. C. Parodi, J. C. Palmaz, and H. D. Barone, "Transfemoral intraluminal graft implantation for abdominal aortic aneurysms," Annals of Vascular Surgery, vol. 5, pp. 491-499, 1991.

[4] K. A. Giles, M. L. Schermerhorn, A. J. O'Malley, P. Cotterill, A. Jhaveri, F. B. Pomposelli, and B. E. Landon, "Risk prediction for perioperative mortality of endovascular vs open repair of abdominal aortic aneurysms using the Medicare population," in 35th Annual Meeting of the New-EnglandSociety-for-Vascular-Surgery, Newport, RI, 2008, pp. 256-262.

[5] K. Liffman, M. M. D. Lawrence-Brown, J. B. Semmens, A. Bui, M. Rudman, and D. E. Hartley, "Analytical modeling and numerical simulation of forces in an endoluminal graft," Journal of Endovascular Therapy, vol. 8, pp. 358-371, Aug 2001.

[6] E. A. Finol, K. G. Marra, and C. H. Amon, "Analytical estimation of flow-induced forces in endovascular grafts and design methodology for a tissue-engineered endovascular attachment mechanism," in 5th International Conference on Computer Simulations in Biomedicine, Ljubljana, Slovenia, 2003, pp. 231-241.

[7] L. Morris, P. Delassus, M. Walsh, and T. McGloughlin, "A mathematical model to predict the in vivo pulsatile drag forces acting on bifurcated stent grafts used in endovascular treatment of abdominal aortic aneurysms (AAA)," Journal of Biomechanics, vol. 37, pp. 1087-1095, Jul 2004.

[8] F. M. White, Fluid Mechanics, 4th edition ed. Boston: McGraw Hill, 1999.

[9] I. V. Mohan, P. L. Harris, C. J. van Marrewijk, R. J. Laheij, and T. V. How, "Factors and forces influencing stent-graft migration after endovascular aortic aneurysm repair," Journal of Endovascular Therapy, vol. 9, pp. 748-755, Dec 2002.

[10] E. A. Finol and C. H. Amon, "Blood flow in abdominal aortic aneurysms: Pulsatile flow hemodynamics," Journal of Biomechanical Engineering-Transactions of the ASME, vol. 123, pp. 474484, Oct 2001.

[11] E. A. Finol, E. S. Di Martino, D. A. Vorp, and C. H. Amon, "Biomechanics of patient specific Abdominal Aortic Aneurysms: Computational analysis of fluid flow," in IEEE 28th Annual Northeast Bioengineering Conference, Philadelphia, Pa, 2002, pp. 191-192.

[12] E. A. Finol, K. Keyhani, and C. H. Amon, "The effect of asymmetry in abdominal aortic aneurysms under physiologically realistic pulsatile flow conditions," Journal of Biomechanical EngineeringTransactions of the ASME, vol. 125, pp. 207-217, Apr 2003.

[13] E. S. Di Martino, G. Guadagni, A. Fumero, G. Ballerini, R. Spirito, P. Biglioli, and A. Redaelli, "Fluid-structure interaction within realistic three-dimensional models of the aneurysmatic aorta as a guidance to assess the risk of rupture of the aneurysm," Medical Engineering \& Physics, vol. 23, pp. 647-655, Nov 2001. 
[14] B. A. Howell, T. Kim, A. Cheer, H. Dwyer, D. Saloner, and T. A. M. Chuter, "Computational fluid dynamics within bifurcated abdominal aortic stent-Grafts," Journal of Endovascular Therapy, vol. 14, pp. 138-143, Apr 2007.

[15] D. S. Molony, A. Callanan, L. G. Morris, B. J. Doyle, M. T. Walsh, and T. M. McGloughlin, "Geometrical Enhancements for Abdominal Aortic Stent-Grafts," Journal of Endovascular Therapy, vol. 15, pp. 518-529, Oct 2008.

[16] T. Frauenfelder, M. Lotfey, T. Boehm, and S. Wildermuth, "Computational fluid dynamics: Hemodynamic changes in abdominal aortic aneurysm after stent-graft implantation," Cardiovascular and Interventional Radiology, vol. 29, pp. 613-623, 2006.

[17] C. M. Scotti, E. A. Finol, and C. H. Amon, "Computational fluid dynamics and wall mechanics of preand post-operative abdominal aortic aneurysms," in 3rd International Conference on Computational Fluid Dynamics, Toronto, CANADA, 2004, pp. 329-334.

[18] C. A. Figueroa, C. A. Taylor, V. Yeh, A. J. Chiou, and C. K. Zarins, "Effect of Curvature on Displacement Forces Acting on Aortic Endografts: A 3-Dimensional Computational Analysis," Journal of Endovascular Therapy, vol. 16, pp. 284-294, Jun 2009.

[19] T. J. Corbett, A. Callanan, L. G. Morris, B. J. Doyle, P. A. Grace, E. G. Kavanagh, and T. M. McGloughlin, "A Review of the In Vivo and In Vitro Biomechanical Behavior and Performance of Postoperative Abdominal Aortic Aneurysms and Implanted Stent-Grafts," Journal of Endovascular Therapy, vol. 15, pp. 468-484, Aug 2008.

[20] Z. Li, C. Kleinstreuer, and M. Farber, "Computational analysis of biomechanical contributors to possible endovascular graft failure," Biomechanics and Modeling in Mechanobiology, vol. 4, pp. 221$234,2005$.

[21] Z. Li and C. Kleinstreuer, "Analysis of biomechanical factors affecting stent-graft migration in an abdominal aortic aneurysm model," Journal of Biomechanics, vol. 39, pp. 2264-2273, 2006.

[22] K. Liffman, I. D. Sutalo, A. Bui, M. M. D. Lawrence-Brown, and J. B. Semmens, "Experimental Measurement and Mathematical Modeling of Pulsatile Forces on a Symmetric, Bifurcated Endoluminal Stent Graft Model," Vascular, vol. 17, pp. 201-209, Jul-Aug 2009.

[23] S. N. Zhou, T. V. How, R. A. Black, S. R. Vallabhaneni, R. McWilliams, and J. A. Brennan, "Measurement of pulsatile haemodynamic forces in a model of a bifurcated stent graft for abdominal aortic aneurysm repair," Proc Inst Mech Eng [H], vol. 222, pp. 543-9, May 2008.

[24] L. Morris, P. Delassus, P. Grace, F. Wallis, M. Walsh, and T. McGloughlin, "Effects of flat, parabolic and realistic steady flow inlet profiles on idealised and realistic stent graft fits through Abdominal Aortic Aneurysms (AAA)," in Meeting on Physical, Mathematical and Numerical Modelling of Blood Flow in Cardiovascular Disease, York, ENGLAND, 2006, pp. 19-26.

[25] H. Sun, B. D. Kuban, P. Schmalbrock, and M. H. Friedman, "Measurement of the geometric parameters of the aortic bifurcation from magnetic-resonance images," Annals of Biomedical Engineering, vol. 22, pp. 229-239, May-Jun 1994.

[26] ANSYS CFX, "Recommended Configurations of Boundary Conditions," in ANSYS CFX-Solver Modeling Guide: ANSYS CFX, Release 11.0 2006, pp. ANSYS CFX-Solver Modeling Guide | Boundary Condition Modeling | Using Boundary Conditions |. 
[27] H. S. Foundation, "Heart and Stroke Foundation of Canada," in http://www.heartandstroke.com/site/c.ikIQLcMWJtE/b.3484023/k.2174/Heart_disease_High_blood_ pressure.htm?gclid=COTO547ospwCFQEhDQod2DtQnw. vol. Accessed March 2009, 2009.

[28] T. McGloughlin, "Personal Communication 2010 Jan 15: Blood velocity profile to be used in AAA simulations," A. Nabovati, Ed., 2010.

[29] ANSYS CFX, "The k-omega and SSTModels " in ANSYS CFX-Solver Modeling Guide: ANSYS CFX, Release 11.0 2006, pp. ANSYS CFX-Solver Modeling Guide | Turbulence and Near-Wall Modeling | Turbulence Models | The k-omega and SSTModels

[30] G. Chatzimavroudis, "Blood Flow Dynamics: 26.4.3 Effect of Flow Pulsatility," in Fluid Flow Handbook, J. M. Saleh, Ed. New York: McGraw Hill, 2002, p. 26.9.

[31] ANSYS Customer Support, "Personal Communication: Question regarding how artificial walls work," L. W. H. Tse, Ed., 2010.

[32] M. Malina, B. Lindblad, K. Ivancev, M. Lindh, J. Malina, and J. Brunkwall, "Endovascular AAA exclusion: Will stents with hooks and barbs prevent stent-graft migration?," Journal of Endovascular Surgery, vol. 5, pp. 310-317, Nov 1998.

[33] T. Resch, M. Malina, B. Lindblad, J. Malina, J. Brunkwall, and K. Ivancev, "The impact of stent design on proximal stent-graft fixation in the abdominal aorta: an experimental study," European Journal of Vascular and Endovascular Surgery, vol. 20, pp. 190-195, Aug 2000.

[34] R. J. Hinchliffe, S. Natarajan, and B. R. Hopkinson, "In vitro analysis of modular aortic stent-graft failure," Journal of Endovascular Therapy, vol. 13, pp. 77-84, Feb 2006.

[35] W. Bosman, T. J. van der Steenhoven, D. R. Suarez, J. W. Hinnen, E. R. Valstar, and J. F. Hamming, "The Proximal Fixation Strength of Modern EVAR Grafts in a Short Aneurysm Neck. An In Vitro Study," European Journal of Vascular and Endovascular Surgery, vol. 39, pp. 187-192, Feb 2010.

[36] D. P. Cina, G. Grant, M. Peterson, V. Campbell, L. Garrido-Olivares, and C. S. Cina, "A Study of Pullout Forces of the Components of Modular Multi-manufacturer Hybrid Endografts Used for Aortic Aneurysm Repair," European Journal of Vascular and Endovascular Surgery, vol. 37, pp. 671-680, Jun 2009.

[37] X. W. Zhang, Z. H. Yao, Y. Zhang, and S. D. Xu, "Experimental and computational studies on the flow fields in aortic aneurysms associated with deployment of AAA stent-grafts," Acta Mechanica Sinica, vol. 23, pp. 495-501, Oct 2007.

[38] H. J. Salacinski, S. Goldner, A. Giudiceandrea, G. Hamilton, A. M. Seifalian, A. Edwards, and R. J. Carson, "The mechanical behavior of vascular grafts: A review," Journal of Biomaterials Applications, vol. 15, pp. 241-278, 2001.

[39] I. E. Vignon-Clementel, C. A. Figueroa, K. E. Jansen, and C. A. Taylor, "Outflow boundary conditions for three-dimensional finite element modeling of blood flow and pressure in arteries," Computer Methods in Applied Mechanics and Engineering, vol. 195, pp. 3776-3796, 2006.

[40] D. S. Molony, E. G. Kavanagh, P. Madhavan, M. T. Walsh, and T. M. McGloughlin, "A Computational Study of the Magnitude and Direction of Migration Forces in Patient-specific Abdominal Aortic Aneurysm Stent-Grafts.," Eur J Vasc Endovasc Surg, vol. Epub ahead of print, pp. $1-8,2010$ Jun 21. 


\section{Appendix I: Derivation of Force Equations}

Newton's Second Law can be written in terms of momentum flux:

$$
\begin{aligned}
\sum \vec{F} & =m \vec{a} \\
& =m \frac{d \vec{v}}{d t} \\
& =\frac{d m \vec{v}}{d t} \\
& =\frac{d \text { Momentum }}{d t}
\end{aligned}
$$

Or, $\sum \vec{F}_{s y s}=\frac{d \vec{M}_{s y s}}{d t}$

$$
\begin{aligned}
& \begin{array}{l}
B=m b \\
\text { Reynolds Transport Theorem says for any quality, B: } \frac{D B_{\text {sys }}}{D t}=\frac{\partial B_{c v}}{\partial t}+B_{\text {out }}-\dot{B}_{\text {in }} \\
\frac{D B_{\text {sys }}}{D t}=\frac{\partial}{\partial t} \int_{c v} \rho b d \forall+\int_{c s} \rho b \vec{V} . \hat{n} d A
\end{array}
\end{aligned}
$$

And if we use Momentum as B, then: $\frac{D \vec{M}_{s y s}}{D t}=\frac{\partial \vec{M}_{c v}}{\partial t}+\vec{M}_{\text {out }}-\dot{\vec{M}}_{\text {in }}$

Therefore, $\sum \vec{F}_{\text {sys }}=\frac{\partial \vec{M}_{c v}}{\partial t}+\vec{M}_{\text {out }}-\dot{\vec{M}}_{\text {in }}$

Or, explained in words,

$$
\begin{aligned}
& {\left[\begin{array}{l}
\text { Sum of forces } \\
\text { on the mass inside } \\
\text { control volume }
\end{array}\right]=\left[\begin{array}{l}
\text { Rate of } \\
\text { accumulation } \\
\text { of momentum } \\
\text { inside } \\
\text { control volume }
\end{array}\right]+\left[\begin{array}{l}
\text { Rate of } \\
\text { momentum } \\
\text { flow leaving } \\
\text { control volume }
\end{array}\right]-\left[\begin{array}{l}
\text { Rate of } \\
\text { momentum } \\
\text { flow entering } \\
\text { control volume }
\end{array}\right]} \\
& \text { For steady flow, }\left(\frac{\partial \vec{M}_{c v}}{\partial t}=0\right)
\end{aligned}
$$


So that $\sum \vec{F}_{\text {sys }}=\dot{\bar{M}}_{\text {out }}-\dot{\bar{M}}_{\text {in }}$

Which can be expanded to $\sum \vec{F}_{\text {sys }}=\int_{\text {Aout }} \rho \vec{V}\left(V_{n}\right) d A-\int_{\text {Ain }} \rho \vec{V}\left(V_{n}\right) d A$

We can also write out that $\sum \vec{F}_{\text {sys }}=\vec{F}_{\text {press }}+\vec{F}_{\text {weight }}+\vec{F}_{\text {reaction }}$

And if we combine the two equations above, we get

$$
\vec{F}_{\text {press }}+\vec{F}_{\text {weight }}+\vec{F}_{\text {reaction }}=\int_{\text {Aout }} \rho \vec{V}\left(V_{n}\right) d A-\int_{\text {Ain }} \rho \vec{V}\left(V_{n}\right) d A
$$

Which can be rearranged and written as

$$
\vec{F}_{\text {reaction }}=-\vec{F}_{\text {press }}-\vec{F}_{\text {weight }}+\int_{\text {Aout }} \rho \vec{V}\left(V_{n}\right) d A-\int_{\text {Ain }} \rho \vec{V}\left(V_{n}\right) d A
$$

Or, $\vec{F}_{\text {anchoring }}=\vec{F}_{\text {press }}+\vec{F}_{\text {weight }}-\left(\int_{\text {Aout }} \rho \vec{V}\left(V_{n}\right) d A-\int_{\text {Ain }} \rho \vec{V}\left(V_{n}\right) d A\right)$

So that we can solve in the $\mathrm{x}$ - and $\mathrm{y}$-directions:

$$
\begin{aligned}
& \vec{F}_{\text {anchoring, } x}=\vec{F}_{\text {press }, x}+\vec{F}_{\text {weight }, x}-\left(\rho v_{2}^{2} A_{2} \sin \theta_{2}-\rho v_{3}^{2} A_{3} \sin \theta_{3}\right)_{\text {m,out }}+\left(\rho v_{1}^{2} A_{1} \sin \theta_{1}\right)_{\text {mi,in }} \\
& =\vec{F}_{\text {press }, x}+\vec{F}_{\text {wight } x}-\left(\rho v_{2}^{2} A_{2} \sin \theta_{2}-\rho v_{3}^{2} A_{3} \sin \theta_{3}\right)_{m_{m, o u t}}+0_{\text {mi, in }} \\
& =\left[\left(-P_{2} A_{2} \sin \theta_{2}+P_{3} A_{3} \sin \theta_{3}\right)_{\text {outlet }}+\left(P_{1} A_{1} \sin \theta_{1}\right)_{\text {inlet }}\right]_{F_{\text {pres } x}}+\vec{F}_{\text {weight } x}-\left(\rho v_{2}^{2} A_{2} \sin \theta_{2}-\rho v_{3}^{2} A_{3} \sin \theta_{3}\right)_{\text {m,out }}+0_{\text {min }, \text { n }} \\
& =\left[\left(-P_{2} A_{2} \sin \theta_{2}+P_{3} A_{3} \sin \theta_{3}\right)_{\text {outlet }}+0_{\text {inlet }}\right]_{F_{\text {pres } x}}+\vec{F}_{\text {weight } x}-\left(\rho v_{2}^{2} A_{2} \sin \theta_{2}-\rho v_{3}^{2} A_{3} \sin \theta_{3}\right)_{\text {mi,out }}+0_{\text {mi, in }} \\
& =\left[\left(-P_{2} A_{2} \sin \theta_{2}+P_{3} A_{3} \sin \theta_{3}\right)_{\text {oulte }}+0_{\text {inlet }}\right]_{F_{\text {pres } x}}+0_{\text {weight } x}-\left(\rho v_{2}^{2} A_{2} \sin \theta_{2}-\rho v_{3}^{2} A_{3} \sin \theta_{3}\right)_{\text {mi,ut }}+0_{\text {minin }} \\
& =\left(-P_{2} A_{2} \sin \theta_{2}+P_{3} A_{3} \sin \theta_{3}\right)_{F_{\text {press } \text { outele. }}}-\left(\rho v_{2}^{2} A_{2} \sin \theta_{2}-\rho v_{3}^{2} A_{3} \sin \theta_{3}\right)_{\text {m,out }} \\
& =P_{3} A_{3} \sin \theta_{3}+\rho v_{3}^{2} A_{3} \sin \theta_{3}-P_{2} A_{2} \sin \theta_{2}-\rho v_{2}^{2} A_{2} \sin \theta_{2} \\
& \vec{F}_{\text {anchoring, }, y}=\vec{F}_{\text {press,y },}+\vec{F}_{\text {wight, },}-\left(\rho v_{2}^{2} A_{2} \cos \theta_{2}+\rho v_{3}^{2} A_{3} \cos \theta_{3}\right)_{\text {m,out }}+\left(\rho v_{1}^{2} A_{1} \cos \theta_{1}\right)_{\text {min }} \\
& =\vec{F}_{\text {press,y }}+\vec{F}_{\text {weight, } y}-\left(\rho v_{2}^{2} A_{2} \cos \theta_{2}+\rho v_{3}^{2} A_{3} \cos \theta_{3}\right)_{\text {m,out }}+\left(\rho v_{1}^{2} A_{1}(1)\right)_{\text {min }} \\
& =\left[\left(-P_{2} A_{2} \cos \theta_{2}-P_{3} A_{3} \cos \theta_{3}\right)_{\text {oulet }}+\left(P_{1} A_{1} \cos \theta_{1}\right)_{\text {inlet }}\right]_{F_{\text {press }}}+\vec{F}_{\text {weight, } y}-\left(\rho v_{2}^{2} A_{2} \cos \theta_{2}+\rho v_{3}^{2} A_{3} \cos \theta_{3}\right)_{\text {mi,out }}+\left(\rho v_{1}^{2} A_{1}\right)_{\text {min }} \\
& =\left[\left(-P_{2} A_{2} \cos \theta_{2}-P_{3} A_{3} \cos \theta_{3}\right)_{\text {outlet }}+\left(P_{1} A_{1}\right)_{\text {intet }}\right]_{F_{\text {rusey }}}+\vec{F}_{\text {weight, } y}-\left(\rho v_{2}^{2} A_{2} \cos \theta_{2}+\rho v_{3}^{2} A_{3} \cos \theta_{3}\right)_{\text {mi,out }}+\left(\rho v_{1}^{2} A_{1}\right)_{\text {min }} \\
& =\left[\left(-P_{2} A_{2} \cos \theta_{2}-P_{3} A_{3} \cos \theta_{3}\right)_{\text {oultet }}+\left(P_{1} A_{1}\right)_{\text {inter }}\right]_{F_{\text {ruses }}}+\vec{F}_{\text {weight,y }}-\left(\rho v_{2}^{2} A_{2} \cos \theta_{2}+\rho v_{3}^{2} A_{3} \cos \theta_{3}\right)_{\text {miout }}+\left(\rho v_{1}^{2} A_{1}\right)_{\text {min }} \\
& =P_{1} A_{1}+\rho v_{1}^{2} A_{1}-P_{2} A_{2} \cos \theta_{2}-\rho v_{2}^{2} A_{2} \cos \theta_{2}-P_{3} A_{3} \cos \theta_{3}-\rho v_{3}^{2} A_{3} \cos \theta_{3}+\vec{F}_{\text {weight, }}
\end{aligned}
$$

And the unknowns are underlined as shown: 


$$
\begin{aligned}
& \vec{F}_{\text {anchoring }, x}=\underline{P_{3}} A_{3} \sin \theta_{3}+\rho \underline{v_{3}^{2}} A_{3} \sin \theta_{3}-\underline{P_{2}} A_{2} \sin \theta_{2}-\rho \underline{v_{2}^{2}} A_{2} \sin \theta_{2} \\
& \vec{F}_{\text {anchoring, } y}=P_{1} A_{1}+\rho v_{1}^{2} A_{1}-\underline{P_{2}} A_{2} \cos \theta_{2}-\rho \underline{v_{2}^{2}} A_{2} \cos \theta_{2}-\underline{P_{3}} A_{3} \cos \theta_{3}-\rho \underline{v_{3}^{2}} A_{3} \cos \theta_{3}+\vec{F}_{\text {weight }, y}
\end{aligned}
$$

In order to solve for the unknowns, we need the following auxiliary equations:

The continuity equation says

$$
\begin{aligned}
\dot{m}_{\text {in }} & =\dot{m}_{\text {in }} \\
\rho Q_{\text {in }} & =\rho Q_{\text {out }} \\
\rho A_{1} \overline{V_{1}} & =\rho A_{2} \bar{V}_{2}+\rho A_{3} \bar{V}_{3} \\
A_{1} \bar{V}_{1} & =A_{2} \bar{V}_{2}+A_{3} \bar{V}_{3}
\end{aligned}
$$

And the modified Bernoulli equation says

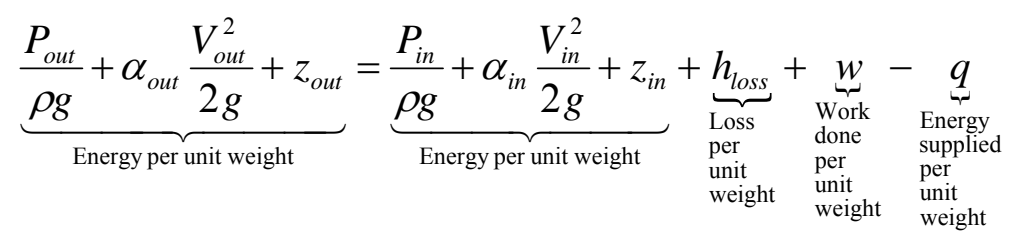

Where head loss can be broken down into $h_{\text {loss }}=\sum h_{\text {major_loss }}+\sum h_{\text {minor_loss }}$

And the Dacy-Weisbach formula says $\underbrace{h_{\text {a }}=f\left(\frac{v^{2}}{2 g}\right)\left(\frac{L}{D}\right)}_{\text {major_loss }}$

Darcy-Weisbach_forumla

And, $h_{\text {minor_loss }}=\xi\left(\frac{v^{2}}{2 g}\right)$

For serially connected pipes, 


$$
\begin{aligned}
h_{\text {loss_serial }} & =h_{\text {loss }, 1}+. .+h_{\text {loss }, n} \\
& =h_{\text {major_loss, }, 1}+h_{\text {minor_loss, },}+. .+h_{\text {major_loss }, n}+h_{\text {minor_loss }, n} \\
& =f_{1}\left(\frac{v_{1}^{2}}{2 g}\right)\left(\frac{L_{1}}{D_{1}}\right)+\sum \xi_{1}\left(\frac{v_{1}^{2}}{2 g}\right)+. .+f_{n}\left(\frac{v_{n}^{2}}{2 g}\right)\left(\frac{L_{n}}{D_{n}}\right)+\sum \xi_{n}\left(\frac{v_{n}^{2}}{2 g}\right)
\end{aligned}
$$

For laminar flow, $f$ is independent of roughness: $f=\frac{64}{R e}$

For $\operatorname{Re}>5000$ and relative roughness $10^{-6}$ to $10^{-2}: f=\frac{0.25}{\left[\log (e / 3.7 D)+\left(5.74 / \mathrm{Re}^{0.9}\right]^{2}\right.}$

And the rest use either $\underbrace{f=\frac{1}{[1.14-2.0 \log (e / D)]^{2}}}_{\text {very_rough_pipe }}$ or $\underbrace{f=\frac{0.3164}{\mathrm{Re}^{1 / 4}}}_{\text {smooth_pipe }}$ 


\section{Appendix II: Comparing model with published analytical results}

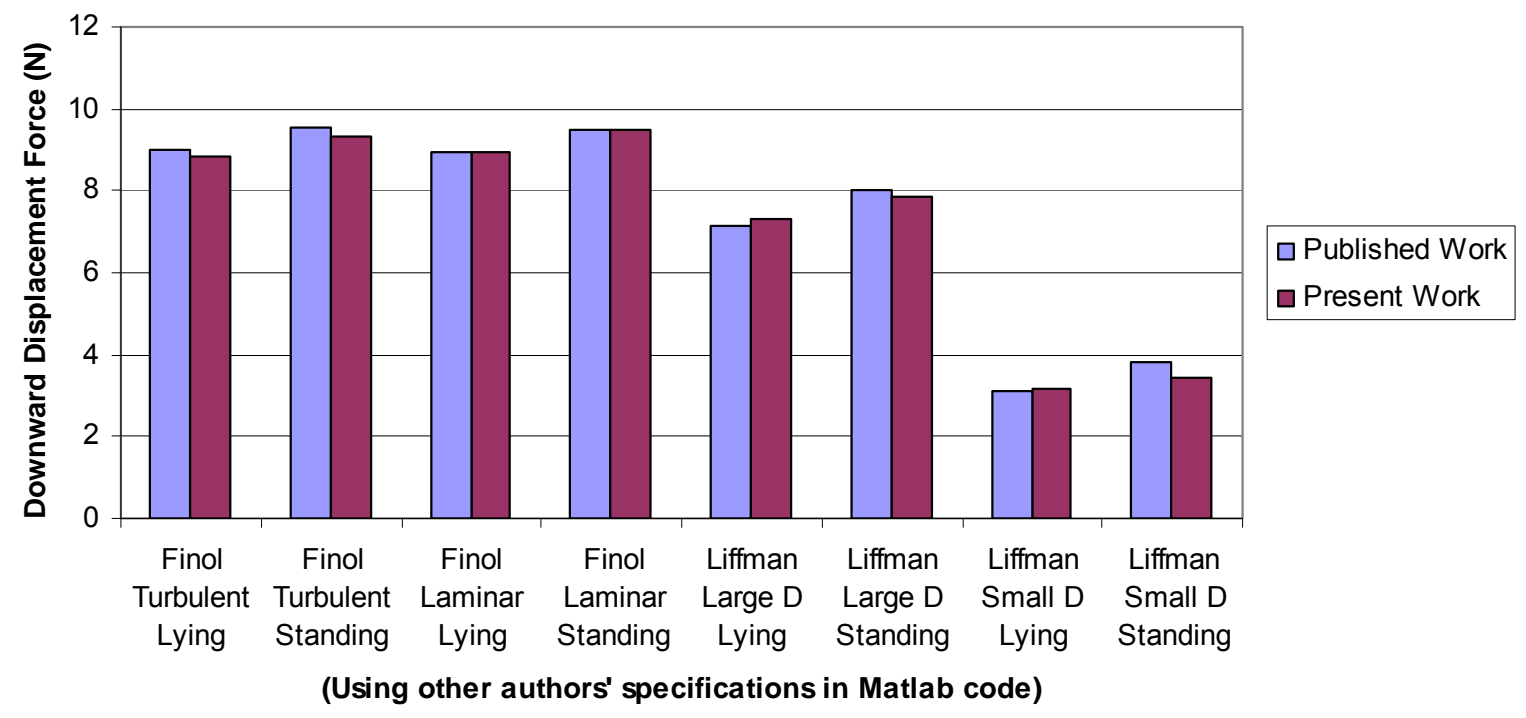

Figure 39: Graph comparing results from Matlab model with published results $[5,6]$. 


\section{Appendix III: Example of mismatched outlet angle}

The following is an example in which a 5-degree difference between the angles of the outlets caused a consistent error in the predicted displacement force for the moderate-redundancy configuration. It is well documented in the literature that changes in the outlet angle have significant effects on the displacement forces [5, 6, 20, 21]. Figure 40 illustrates the geometric difference in the angles, while Fig. 41 is a plot of the predicted displacement forces for the zero-, moderate-, and severe-redundancy configurations (where the moderateredundancy configuration was erroneous).

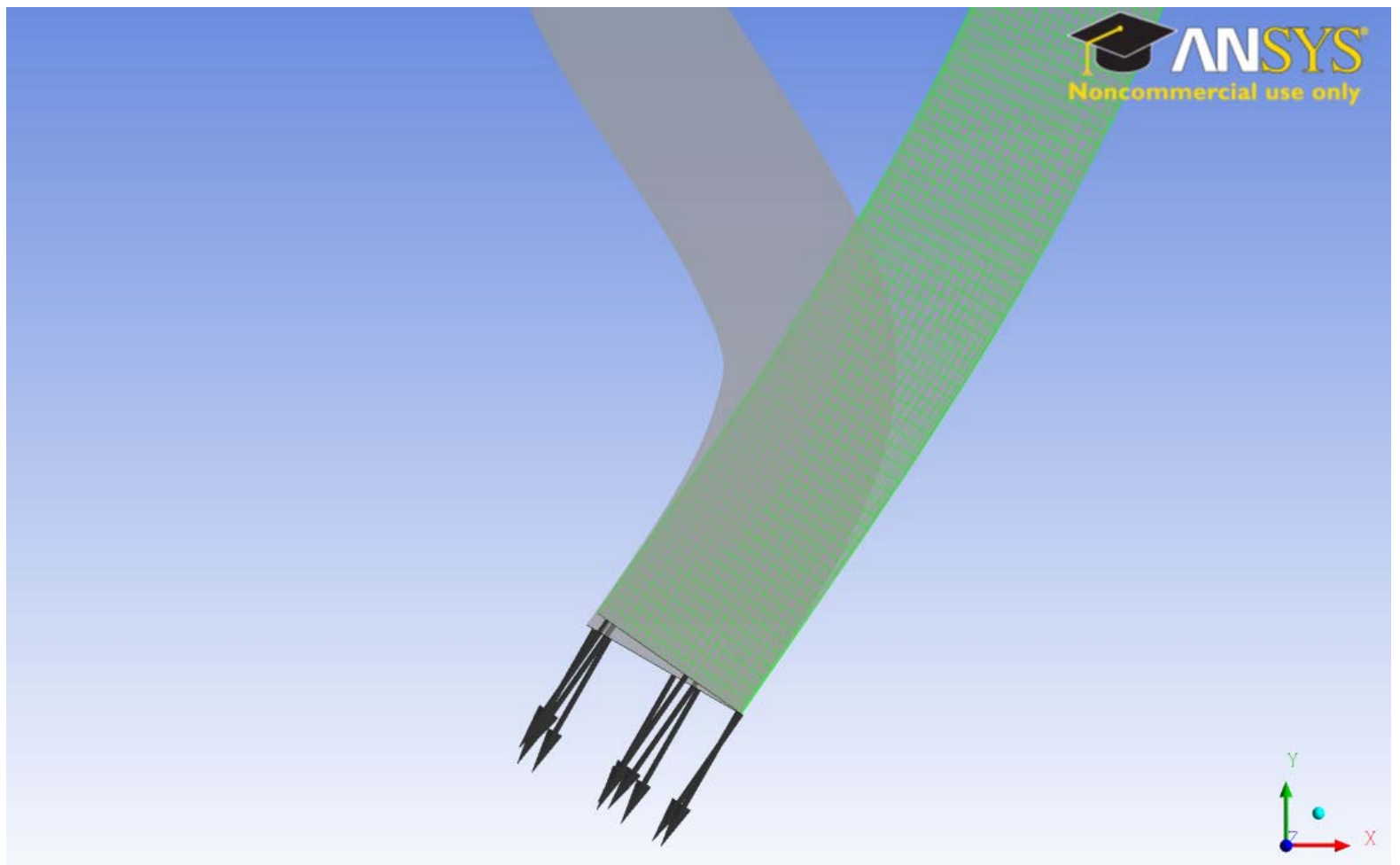

Figure 40: Example of $5^{\circ}$ difference in angle of outlet. The zero-redundancy limb (green) is overlapped onto the erroneous moderate-redundancy limb (grey) 


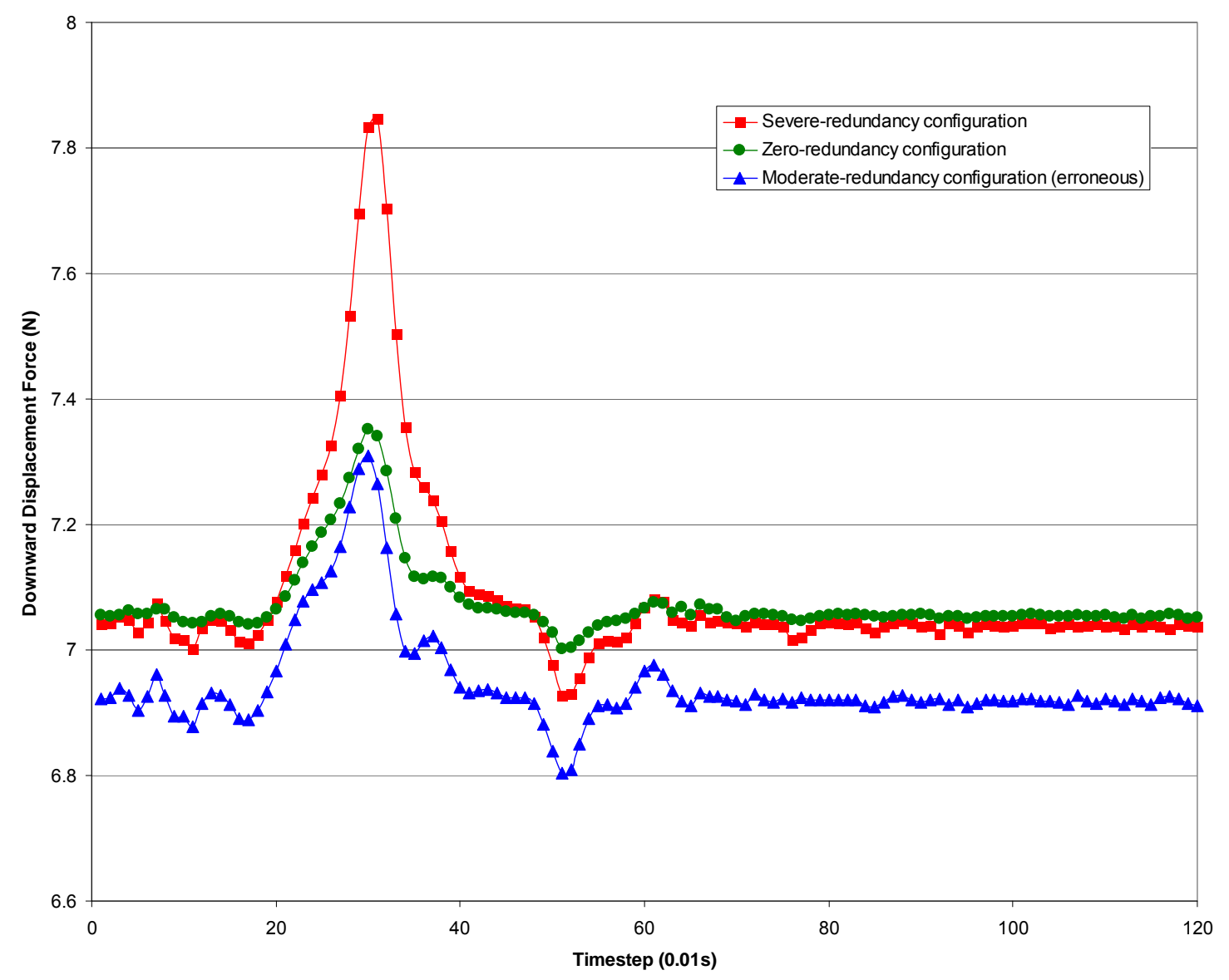

Figure 41: Plot of downward displacement force over time. Note the moderateredundancy configuration (blue triangles) is consistently lower throughout the cardiac cycle.

After correction of the geometry, as shown in Fig. 42, the predicted results were more consistent, as demonstrated in Fig. 27 of the main text in which there is minimal difference between the three configurations during the late diastolic phase of the cardiac cycle. This example serves to emphasize the importance of attention to detail when working with computational fluid dynamics. 


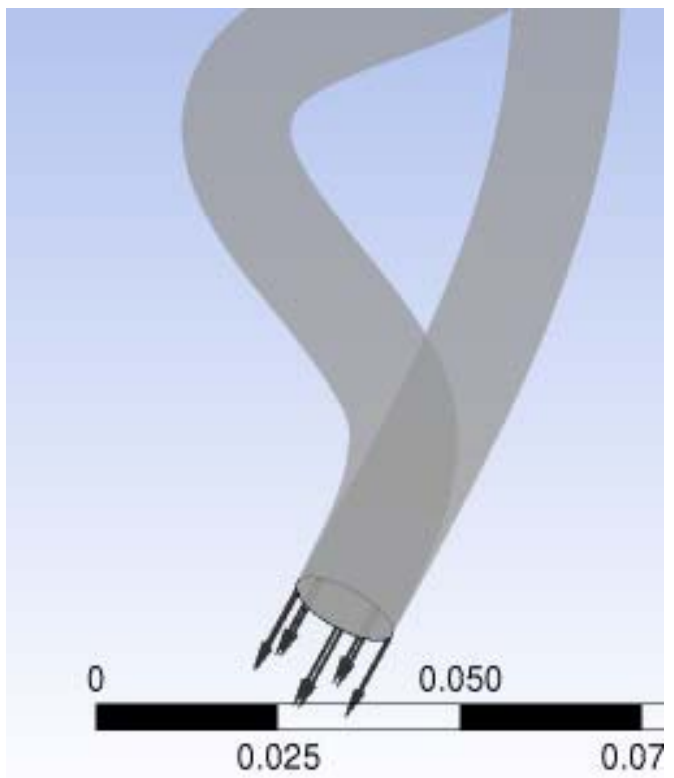

Figure 42: $\quad$ Outlets re-aligned. 